\title{
Friction, Wear and Mechanical Behavior of Nano-Objects on the Nanoscale
}

\section{DISSERTATION}

Presented in Partial Fulfillment of the Requirements for the Degree Doctor of Philosophy in the Graduate School of The Ohio State University

\author{
By \\ Dave Maharaj \\ Graduate Program in Mechanical Engineering \\ The Ohio State University \\ 2015 \\ Dissertation Committee: \\ Professor Bharat Bhushan, Advisor \\ Professor Gary Kinzel \\ Professor Anthony Luscher \\ Professor Erdal Ozkan \\ Professor Shaurya Prakash
}




\begin{abstract}
Nano-objects have been investigated for applications that include drug delivery for cancer treatment, oil detection, contaminant removal, catalysis, and tribology on the macro- to nanoscale. In some applications, they are subjected to friction and deformation during contact with each other and the surfaces on which they are used. Pioneering studies are performed to gain a fundamental understanding of friction and wear of nanoobjects, their mechanical properties and the deformation mechanisms. These unique set of studies examined friction mechanisms in single and multiple nano-object contact sliding on a surface in dry and submerged in liquid environments and sliding on a single nano-object. Mechanical behavior is also investigated. The nano-object contact and friction studies are done using an atomic force microscope (AFM). Single nano-object contact studies, where nano-objects are pushed laterally, provides understanding of friction mechanisms of nano-objects sliding on surfaces. Results of experiments done for the first time, show friction is influenced by real area of contact, roughness, work of adhesion and viscosity of liquids. Friction is lower in liquid environments compared to dry environments. Multiple nano-object contact studies investigate the ability of several nano-objects to reduce friction and wear between sliding surfaces as a result of lower real area of contact. Further studies on friction, sliding on a single nano-object, reveals a dependence on topography, scale and material. Studies are done on local deformation


(indentation) and global deformation (compression) with a sharp tip and flat punch respectively to understand mechanical behavior and are reported for the first time. Local deformation studies allow for investigation of scale effects on mechanical properties. Global deformation studies investigate the occurrence of reverse plasticity and resistance to deformation due to repeat loading.

In this thesis the results of these studies are presented to help understand fundamental interfacial interactions and deformation mechanisms. Studies reported use gold ( $\mathrm{Au}$ ) nanoparticles (NPs) and nanorods (NRs), molybdenum disulfide $\left(\mathrm{MoS}_{2}\right)$ and tungsten disulfide $\left(\mathrm{WS}_{2}\right)$ multi-walled nanotubes (MWNTs) and carbon nanohorns (CNHs). These nano-objects were selected to determine methodology and trends, which guide in the selection of nano-objects for various applications. 


\section{Dedication}

This document is dedicated to my parents

Ramsaran and Taramatee Maharaj

and my sisters

Aruna and Reena Maharaj 


\section{Acknowledgments}

First, I would like to thank my advisor, Professor Bharat Bhushan, who provided countless hours of guidance and detailed feedback on research and writing papers. His tremendous knowledge, dedication to perfection and insight was responsible for helping me to accomplish my research goals. I would like to thank Manuel Palacio for the countless hours of training and scientific discussion he provided. I would also like to thank my lab associates, especially Gregory Bixler, Emre Demirocak, Phillip Brown and Renee Ripley for their support in research and other lab activities. I would also like to thank my doctoral committee members, Professor Gary Kinzel, Professor Anthony Luscher and Professor Shaurya Prakash at The Ohio State University.

I would also like to express gratitude to all family members who provided support in one form or another. Finally I would like to express my sincerest love and gratitude to my parents and my two sisters who provided encouragement and support throughout my academic life. 


\section{Vita}

2006
B.S. Mechanical Engineering, City College

of the City University of New York

..M.E. Mechanical Engineering, City College

of the City University of New York

2009 to present Graduate Research Associate, The Ohio

State University

Journal Publications

1. Maharaj, D. and Bhushan, B. (2012), "Effect of spherical Au nanoparticles on nanofriction and wear reduction in dry and liquid environments," Beilstein J. Nanotechnol. 3, 759-772.

2. Maharaj, D. and Bhushan, B. (2013), "Effect of $\mathrm{MoS}_{2}$ and $\mathrm{WS}_{2}$ nanotubes on nanofriction and wear reduction in dry and liquid environments," Tribol. Lett. 49, 323-339.

3. Maharaj, D., Bhushan, B. and Iijima, S. (2013), "Effect of Carbon Nanohorns on nanofriction and wear reduction in dry and liquid environments," J. Colloid Interface Sci. 400, 147-160

4. Maharaj, D. and Bhushan, B. (2014), "Scale effects of nanomechanical properties and deformation behavior of Au nanoparticle and thin film using depth sensing nanoindentation," Beilstein J. Nanotechnol. 5, 822-836.

5. Maharaj, D. and Bhushan, B. (2014), "Nanomanipulation, nanotribology and nanomechanics of Au nanorods in dry and liquid environments using an AFM and depth sensing nanoindenter," Nanoscale, 6, 5838-5852. 
6. Maharaj, D. and Bhushan, B. (2015), "Characterization of nanofriction of $\mathrm{MoS}_{2}$ and WS 2 nanotubes," Mater. Lett. (In press).

7. Maharaj, D. and Bhushan, B. (2015), "Nanomechanical behavior of $\mathrm{MoS}_{2}$ and $\mathrm{WS}_{2}$ multi-walled nanotubes and Carbon nanohorns," Nature - Scientific Reports.

8. Maharaj, D. and Bhushan, B. (2015), "Friction, wear and mechanical behavior on the nanoscale," In review

\section{Book Chapters}

1. Maharaj, D. and Bhushan, B., (2014), Handbook of Nanomaterials Properties, Bhushan, B., Luo, Dan., Schricker, S. R., Sigmund,W. and Zauscher, S. (Eds.), Springer, Heidelberg, Germany, pp 299-315.

\section{Fields of Study}

Major Field: Mechanical Engineering 


\section{Table of Contents}

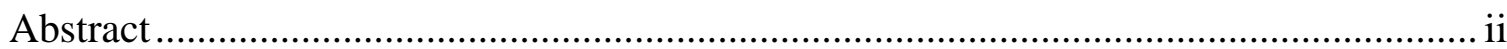



Acknowledgments........................................................................................................ V

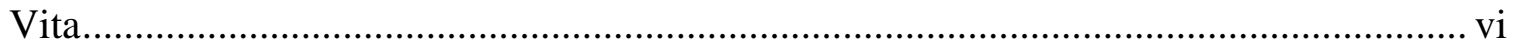

Fields of Study ……...................................................................................... vii

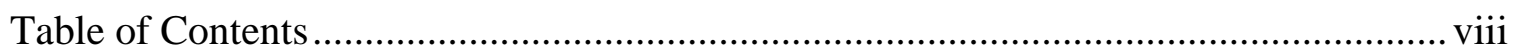

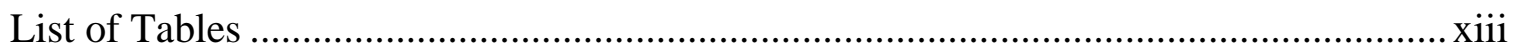

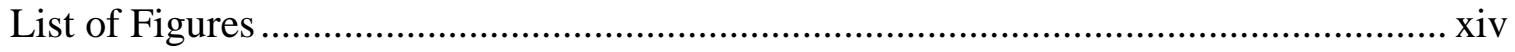

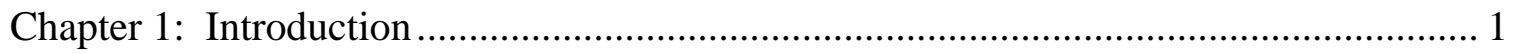



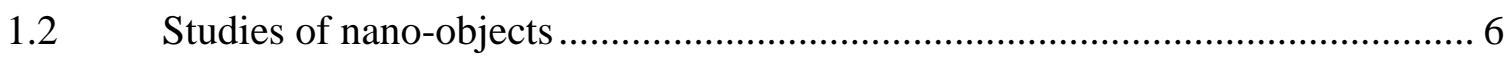

1.2.1. Single nano-object contact studies ........................................................... 7

1.2.2 Multiple nano-object contact studies …………......................................... 9

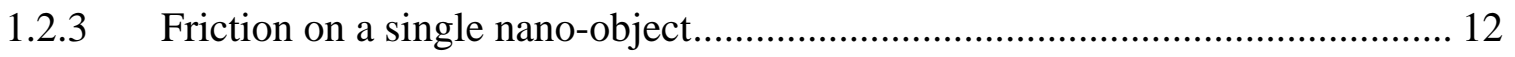

1.2.4 Mechanical behavior of nano-objects and thin films ....................................... 13

1.3 Choice of nano-objects and liquids for this study .......................................... 15 viii 


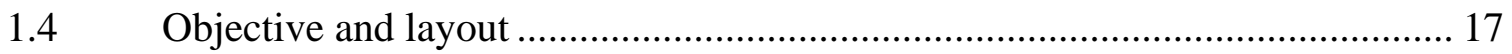



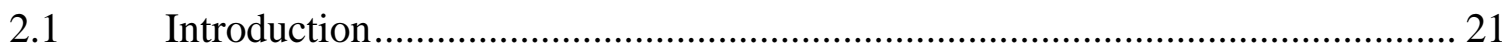

Indentation size effect-Strain gradient plasticity ........................................ 22

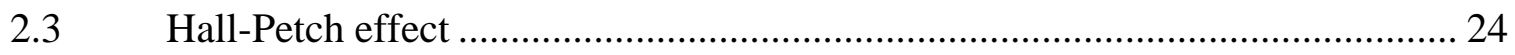

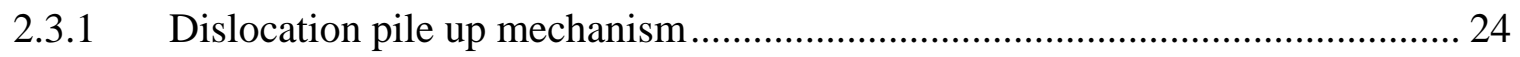



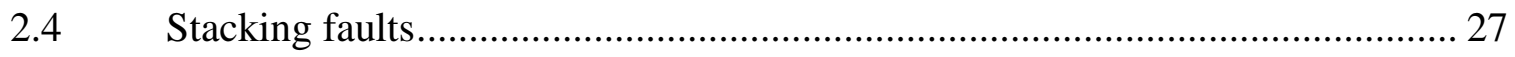



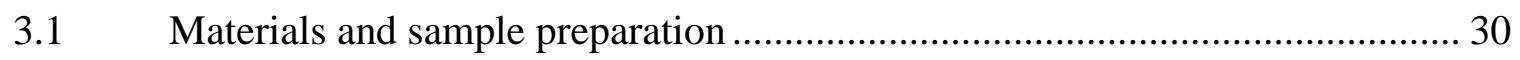

3.2 Friction and wear in dry and liquid environments: Au NPs and NRs, CNHs and

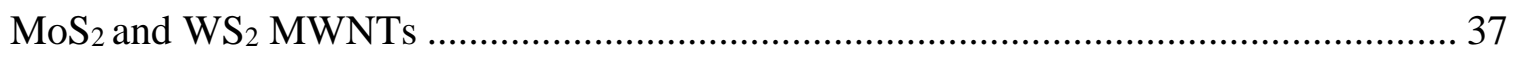

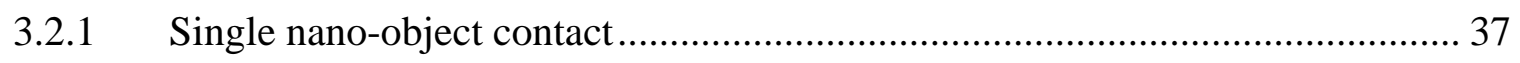

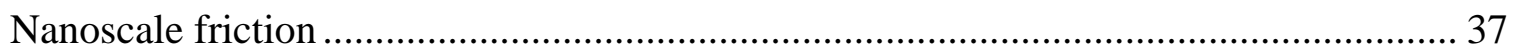

3.2.2 Multiple nano-object contact ............................................................ 38



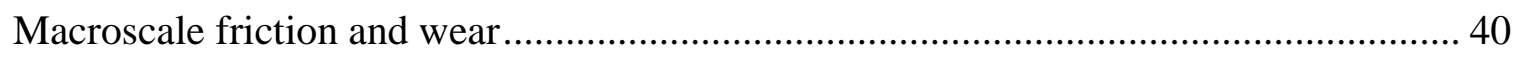

F.3 Friction on single nano-objects: $\mathrm{MoS}_{2}$ and $\mathrm{WS}_{2}$ MWNTs............................... 41 
3.4 Mechanical behavior: Au thin film, NPs and NRs, CNHs and $\mathrm{MoS}_{2}$ and $\mathrm{WS}_{2}$ MWNTs 42

3.4.1 Nanoindentation with a Berkovich tip .................................................... 42

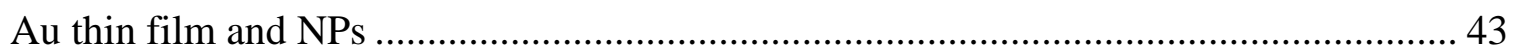

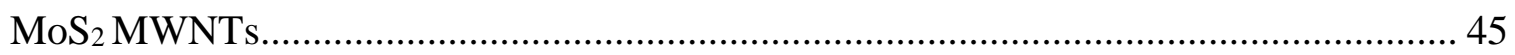

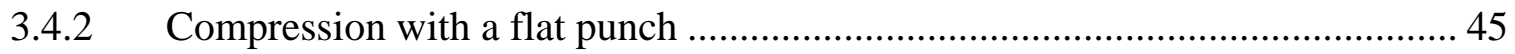

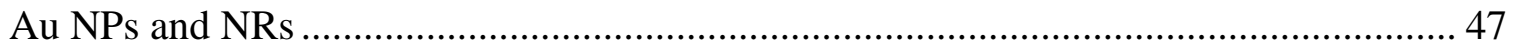

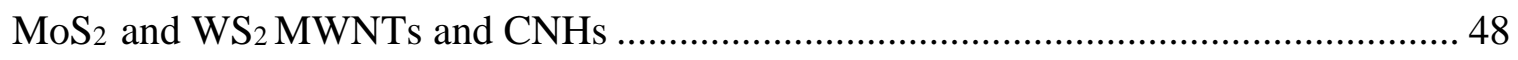

Chapter 4: Friction and wear in dry and liquid environments: Au NPs and NRs........... 49

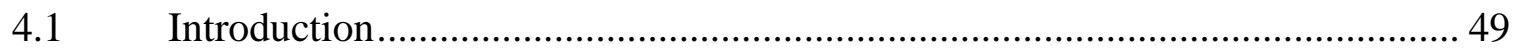

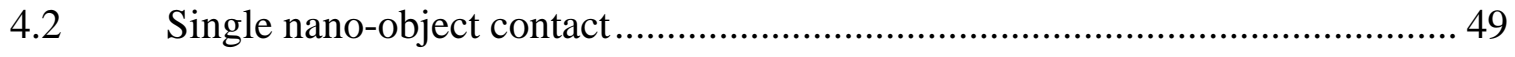

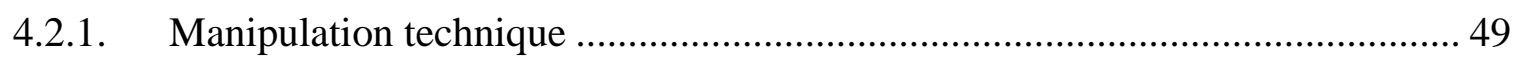

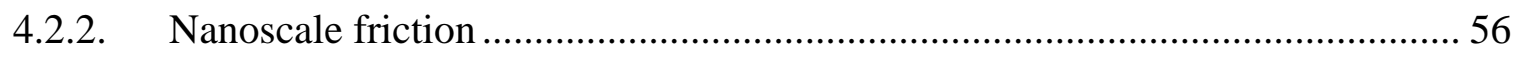

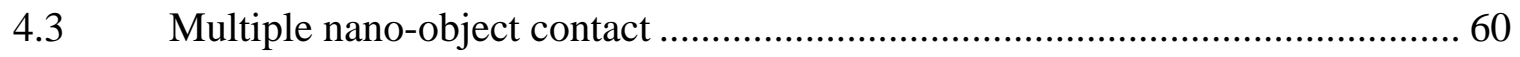

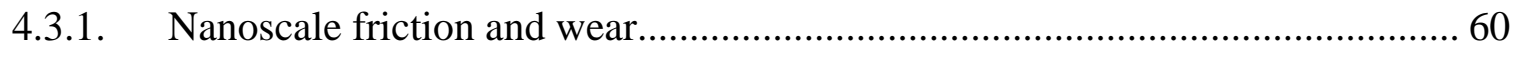

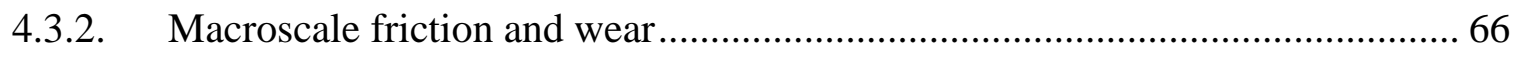

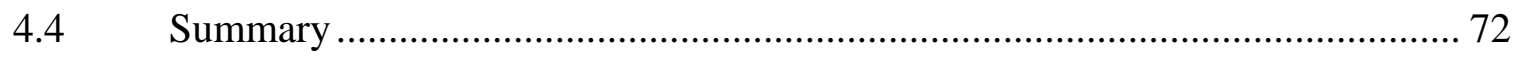

Chapter 5: Friction and wear in dry and liquid environments: Au NPs and NRs, CNHs

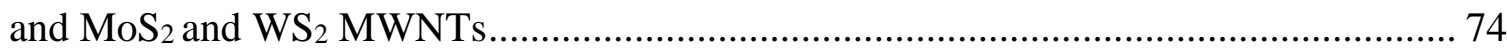




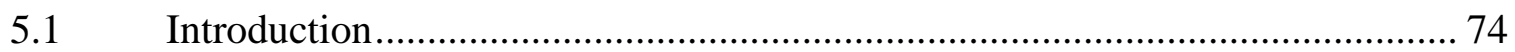

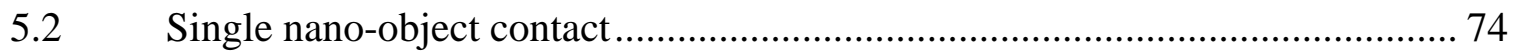

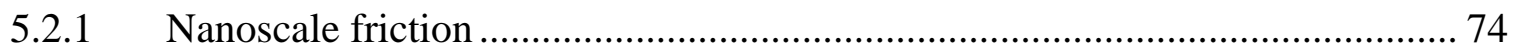



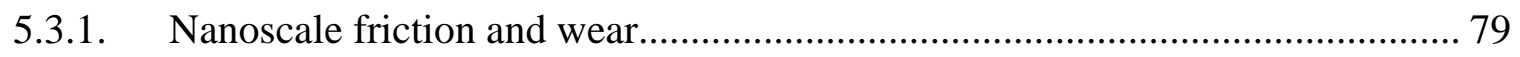



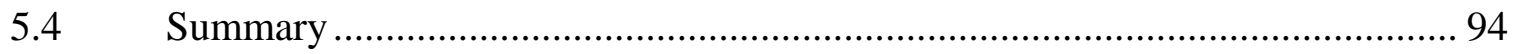

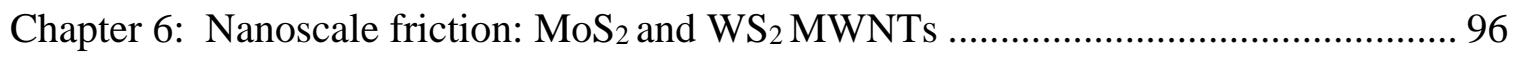

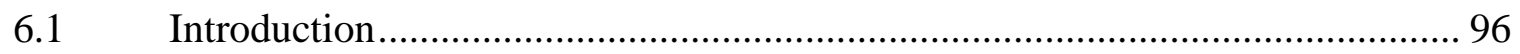

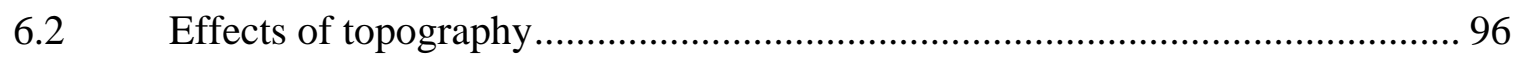

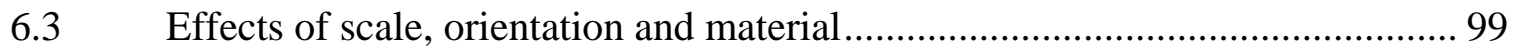

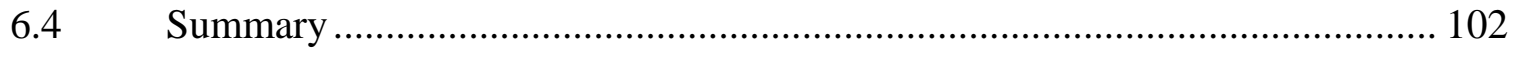

Chapter 7: Mechanical behavior: Au thin film, NPs and NRs................................... 103

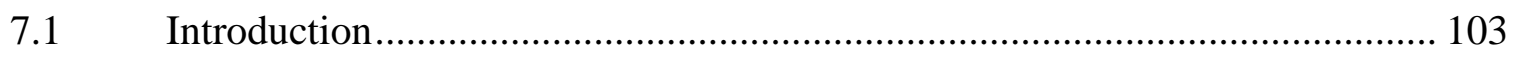

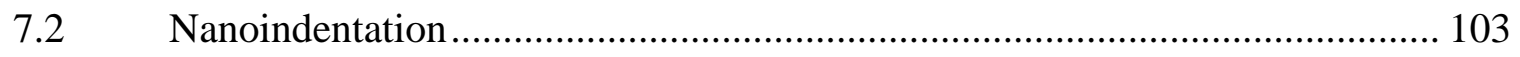

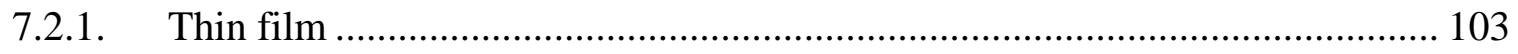

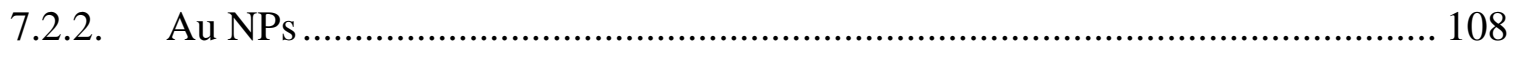

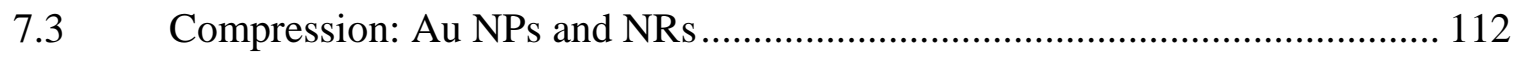

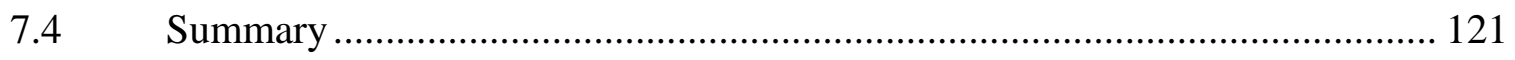


Chapter 8: Mechanical behavior: Au NPs and NRs, CNHs and $\mathrm{MoS}_{2}$ and $\mathrm{WS}_{2} \mathrm{MWNTs}$

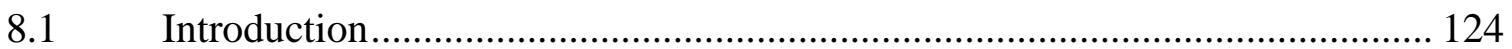

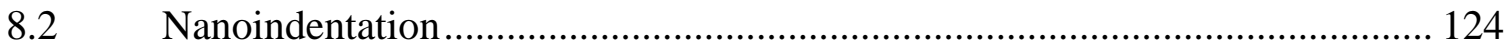

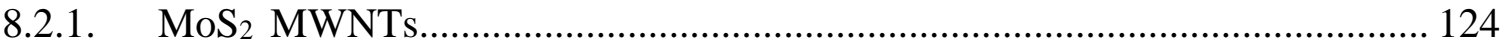

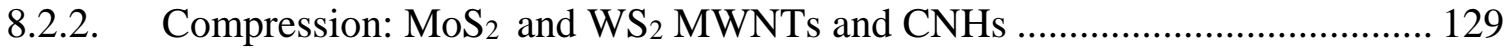

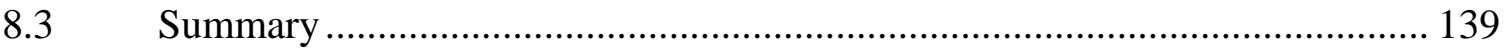

Chapter 9: Conclusions and Outlook .................................................................. 141

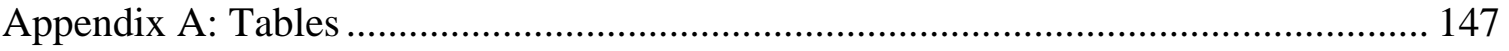

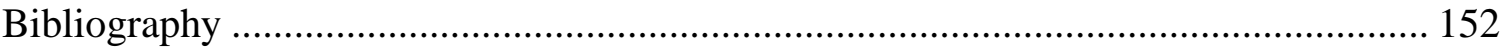




\section{List of Tables}

Table A.1: Review of studies of enhanced scale-dependent mechanical properties of bulk solid surfaces, thin films and various nano-objects................................... 149

Table A.2: Mechanical properties of borosilicate glass, diamond, sapphire and gold. .. 150

Table A.3: Work of adhesion $\left(W_{a}\right)$ for thin films of $\mathrm{Au}, \mathrm{MoS}_{2}, \mathrm{WS}_{2}$ and graphene..... 150

Table A.4: Nanomechanical properties of Au nanoparticles and thin film. ................. 151

Table A.5: Mechanical properties of $\mathrm{MoS}_{2} \mathrm{MWNTs}$ obtained from the nanoindentation experiments normal to the basal plane......................................................... 151 


\section{List of Figures}

Figure 1: Examples of nano-objects in applications in (a) drug delivery, where nanoobjects coated with a drug, targets cancer cells and release their therapeutic payload resulting in death of the cancer cell (Tran and Webster, 2010), (b) oil detection, where carbon black nano-objects are coated with an oil detecting agent. After injection into the ground, the agent is released on contact with hydrocarbons and this is used as an indication of the presence of oil on recovery and analysis of the nanoparticle (Berlin et al., 2011), (c) contaminant removal with magnetic cross-linked knedel-like nanoparticles (MSCKs). The MSCKs are added to the polluted water (left), where they selectively absorb the oil (center) and are finally removed by introduction of a neodymium magnet (Pavia-Sanders et al., 2013) and (d) catalysis for hydrogen evolution recovery (HER), using nanowires. The nanowires consist of an inner $\mathrm{MoO}_{3}$ core and outer $\mathrm{MoS}_{2}$ shell. The core is used for transporting electrons to edge sites of the inner $\mathrm{MoS}_{2}$ shell which is catalytically active. At these sites the transfer of electrons necessary for recovery of hydrogen from water is enhanced by the $\mathrm{MoS}_{2}$, which improves the efficiency of the HER (Chen et al., 2011).

Figure 2: Friction reduction occurs as a result of weak van der Waals forces between layers which allows for easy shearing of the layers (Maharaj and Bhushan, 2013a)...... 11

Figure 3: Illustration of the studies in friction, wear and mechanical behavior of nanoobjects. The studies examined were single and multiple nano-object contact in dry and liquid environments, friction on single nano-objects and mechanical behavior of nanoobjects during indentation and compression (Left column). Observations for each study are given in the column on the right.

Figure 4: Flowchart illustrating dislocation sources, in a grain, from the grain boundary and grain interior, through multiplication and growth of an existing dislocations and from geometrically necessary dislocations needed to accommodate strain gradient (Maharaj and Bhushan, 2014a).

Figure 5: Illustration of the strain gradient plasticity theory where high strain gradients occur at shallow indentation depths with a sharp tip. 
Figure 6: Illustration of (a) Hall-Petch (Based on Hall, 1951; Petch, 1953) effect by dislocation pile up mechanism (Based on Li, 1969; Jonas, 1970), where dislocations pile up against the grain boundary under an applied stress $\tau$. The stress at the boundary $\tau_{\mathrm{p}}$ (pile up stress) which is a function of $\tau$ and $\tau_{\mathrm{i}}$ (internal stress) of the dislocations is larger for a bigger grain size $(\mathrm{A})$ on the macroscale due to the larger number of dislocations compared to that for a smaller grain size $(\mathrm{C})$ on the nanoscale. The number of dislocations (n) within the grain on the nanoscale is smaller compared to the macroscale, requiring a larger $\tau$ to create a pile up stress $\left(\tau_{\text {critical }}\right)$ high enough for dislocation slip and plastic deformation which results in a higher yield stress and (b) Dislocation density mechanism where there is a larger overall grain boundary area as grains become smaller within the same volume, resulting in a greater density of dislocations which impede the formation and motion of new dislocations to accommodate strain gradients and results in higher yield stress (Based on Ashby, 1970).

Figure 7: Schematic showing (a) stacking faults resulting from the removal of a plane of atoms with sessile Frank partials dislocations in the region between the faulted and perfect crystal. The layer directly above the missing atoms changes internally from a ' $\mathrm{B}$ to $\mathrm{B}$ ' to ' $\mathrm{B}$ to $\mathrm{C}$ ' arrangement to prevent an energetically unstable arrangement. The subsequent layer changes from an 'A to A' to 'A to B' arrangement, and (b) stacking faults with glissile Shockley partials in the region between the faulted and perfect crystal. These faults are as a result of an insertion of a plane of atoms with ' $\mathrm{C}$ ' arrangement between a layer with an ' $\mathrm{A}$ ' arrangement. The ' $\mathrm{C}$ ' arrangement is more energetically favorable to a 'B' arrangement (Based on Hull and Bacon, 2001)................................. 29

Figure 8: (a) TEM images of (a) spherical Au nanoparticles approximately $50 \mathrm{~nm}$ in diameter (b) Au nanorods $50 \mathrm{~nm}$ in diameter and $200 \mathrm{~nm}$ in length (Nanopartz.com, 2015).

Figure 9: (a) TEM images of (a) spherical Au nanoparticles approximately $50 \mathrm{~nm}$ in diameter (b) Au nanorods $50 \mathrm{~nm}$ in diameter and $200 \mathrm{~nm}$ in length (Nanopartz.com, 2015).

Figure 10: TEM images of (a) $\mathrm{MoS}_{2} \mathrm{MWNT}$ (Top), a magnified image showing separated layers between the arrows (Left), magnified image showing broken surface layers (Center) and a magnified image of a nanotube showing stacking faults highlighted by the dashed lines (Right) (b) agglomerated WS 2 MWNTS (Left), a magnified image with an arrow pointing to exfoliated layers (Center) and a magnified image of a nanotube showing ordered layers (Maharaj and Bhushan, 2015b). (c) Spherical CNHs (Left) with magnified image of CNHs (Center) and magnified image showing individual horns (Iijima et al., 1999).

Figure 11: Topography map (top) and 2-D profile (bottom) of flat punch at the section shown by the pairs of horizontal arrows along with a typical line plot (right) of a $200 \mathrm{x}$ 
$200 \mathrm{~nm}$ section indicated by the single horizontal arrow (Maharaj and Bhushan, 2014a).

Figure 12: Example of topography maps and height profiles, at sections shown by the arrows, of a Au nanoparticle $90 \mathrm{~nm}$ in diameter, highlighted by the squares, manipulated within the same scan area before and after manipulation (Maharaj and Bhushan, 2012). 50

Figure 13: (a) An example of a topography image and height profile showing the same scan area before and after manipulation (b) Topography maps and 2-D friction force profiles, of a Au $90 \mathrm{NP}$ during manipulation of single nanoparticles in air (dry environment). During manipulation, the normal load is increased (A-B) halfway through the scan of the nanoparticle (indicated by the black arrows) from $10 \mathrm{nN}$ to $300 \mathrm{nN}$ on a single scan line. On this scan line, the sharp tip slides laterally (B-C) until contact is made with the particle. The lateral friction force (C-D) increases, as the sharp tip pushes against the particle, until it begins to move. E shows the end of the scan line (Maharaj and Bhushan, 2012).

Figure 14: Topography map of a Au 90 NP submerged in water. Imaging is performed using a cantilever with a low normal load of $1 \mathrm{nN}$. The highlighted particle is examined and the associated topography and friction force scan lines before manipulation, during manipulation and after manipulation are shown. Before manipulation, there is a rise in friction force corresponding to the nanoparticle profile as the cantilever deflects during imaging. During manipulation the topography profile is flat as the particle is being pushed which corresponds to a rise in friction force (A-B). After manipulation, the topography and friction force profiles remain flat as the particle is no longer there (Maharaj and Bhushan, 2012).

Figure 15: Friction force in (a) air during manipulation of Au 90 NPs, at normal loads of $150 \mathrm{nN}$ and $300 \mathrm{nN}$ (Left) (Maharaj and Bhushan, 2012) and Au 50 NPs and NRs at normal loads of $300 \mathrm{nN}$ on the silicon substrate (Right) (Adapted from Maharaj and Bhushan, 2014b. (b) In water at a normal load of $1 \mathrm{nN}$ for Au 90 NPs, (Left) (Maharaj and Bhushan, 2012) and Au 50 NPs and NRs on the silicon substrate (Right) (Maharaj and Bhushan, 2014b). The vertical scale is magnified for the data in water. (c) Coefficients of friction on the nanoscale for both dry and submerged in water environments, with and without nano-objects for (a) Au 30 and Au 90 (Left) (Maharaj and Bhushan, 2012) and Au 50 NPs and NRs (Right) (Maharaj and Bhushan, 2014b). Friction forces and coefficient of friction are lower in submerged in water environments (Maharaj et al., 2013).

Figure 16: Schematic of friction and wear reducing mechanism of nano-object coated surface by (a) rolling, (b) sliding over nano-objects, (c) dragging of nano-objects, (d) exfoliation, (e) and tribofilm formation. CNHs are used as an example for mechanisms (a) through (c) and $\mathrm{WS}_{2}$ and $\mathrm{MoS}_{2}$ MWNTs are used as an example for mechanisms (d) through (e) (Maharaj et al., 2013). 
Figure 17: Topography maps and 2-D profiles for nanoscale wear experiments, at sections shown by the arrows, after sliding at 1,10 and 100 cycles with a normal load of $20 \mu \mathrm{N}$ at $10 \mathrm{~Hz}$ on Si and Si coated with Au 50 NPs and NRs (Maharaj and Bhushan, 2014b).

Figure 18: SEM micrographs of nanoscale wear scars for Au 50 NPs and NRs in dry conditions after 100 cycles at a normal load of $20 \mu \mathrm{N}$ (First row). The nano-objects within the squares are magnified and the vertical arrows point to those micrographs in the second row. The magnified micrographs show agglomerated Au 50 NPs and NRs as indicated by arrows within the micrographs (Maharaj and Bhushan, 2014b).

Figure 19: (a) Optical micrographs of macroscale wear scars taken after 500 cycles at a normal load of $200 \mathrm{mN}$, for friction tests, in dry and water environments with and without Au 50 NPs and NRs. The sliding speed was $3.5 \mathrm{~mm} / \mathrm{s}$. (b) SEM micrographs of the wear scar for agglomerated Au 50 NPs and NRs in dry conditions shown within the squares (Maharaj and Bhushan, 2014b). The least wear occurs with nano-objects in water.

Figure 20: Macroscale coefficient of friction data after 500 cycles at a normal load of 200 $\mathrm{mN}$, in dry and water conditions with and without Au 50 NPs and NRs (Maharaj and Bhushan, 2014b). The lowest coefficient of friction occurs with Au 50 NPs in water.... 70

Figure 21: Friction force for Au 90 NPs, CNHs and $\mathrm{MoS}_{2}$ and $\mathrm{WS}_{2} \mathrm{MWNT}_{\text {s }}$ on the silicon substrate during manipulation, at normal loads of (a) $300 \mathrm{nN}$ in air and (b) $1 \mathrm{nN}$ in liquids. The vertical scale is magnified for data in liquids. (c) Coefficients of friction for both dry and submerged in liquid environments, with and without nano-objects. Friction forces and coefficient of friction are lower in submerged in water environments (Maharaj et al., 2013). 76

Figure 22: Topography maps and 2-D profiles, at sections shown by the arrows, after sliding at 1, 10 and 100 cycles with a normal load of $20 \mu \mathrm{N}$ on Si and Si coated with Au 90 NPs, CNHs and $\mathrm{MoS}_{2}$ and $\mathrm{WS}_{2}$ nanotubes (Maharaj et al., 2013).

Figure 23: SEM micrographs of nanoscale wear scars for Au 90 NPs, CNHs and $\mathrm{MoS}_{2}$ and $\mathrm{WS}_{2} \mathrm{MWNT}$ in dry conditions after 100 cycles at a normal load of $20 \mu \mathrm{N}$ (First row). The nano-objects within the squares are magnified and the vertical arrows point to those micrographs in the second row. The magnified micrographs show agglomerated $\mathrm{Au}$ 90 NPs and CNHs and broken $\mathrm{MoS}_{2}$ and $\mathrm{WS}_{2}$ MWNTs as indicated by arrows within the micrographs (Maharaj et al., 2013)

Figure 24: Optical micrographs of macroscale wear scars taken after 500 cycles at a normal load of $200 \mathrm{mN}$, for friction tests, in dry, water, dodecane and glycerol conditions with and without $\mathrm{Au} 90 \mathrm{NPs}, \mathrm{CNHs}$ and $\mathrm{MoS}_{2}$ and $\mathrm{WS}_{2}$ nanotubes (Maharaj et al., 2013). The lowest wear occurs in glycerol with CNHs 
Figure 25: Magnified (a) optical micrographs inside the wear scar on the macroscale taken after 500 cycles, at a normal load of $200 \mathrm{mN}$, in dry, water, dodecane and glycerol conditions with and without Au 90 NPs, CNHs and MoS 2 and $\mathrm{WS}_{2}$ MWNTs and (b) SEM micrographs of the wear scar for agglomerated Au 90 NPs, CNHs along with crushed or broken $\mathrm{MoS}_{2}$ and $\mathrm{WS}_{2} \mathrm{MWNTs}$ in dry environments shown within the squares (Maharaj et al., 2013 Maharaj et al., 2013).

Figure 26: Macroscale coefficient of friction data after 500 cycles at a normal load of 200 $\mathrm{mN}$, in dry, water, dodecane and glycerol envionments with and without Au 90 NPs, CNHs and $\mathrm{MoS}_{2}$ and $\mathrm{WS}_{2}$ MWNTs (Maharaj et al., 2013). The lowest wear occurs in glycerol with CNHs.

Figure 27: Examples of topography and friction maps of $\mathrm{MoS}_{2}$ (Left) and $\mathrm{WS}_{2}$ nanotubes (Right) with arrows pointing to magnified images of the areas highlighted by dashed squares (Maharaj and Bhushan, 2015a).

Figure 28: Examples of topography and friction maps of $\mathrm{MoS}_{2}$ (Top) and $\mathrm{WS}_{2} \mathrm{MWNTs}$ (Bottom) with arrows pointing to the sections on which the corresponding profiles were taken. For $\mathrm{MoS}_{2}, \mathrm{RMS}=0.16 \mathrm{~nm}$ and $\mathrm{P}-\mathrm{V}=1.2 \mathrm{~nm}$ and for $\mathrm{WS}_{2}, \mathrm{RMS}=0.12 \mathrm{~nm}$ and $\mathrm{P}-$ $\mathrm{V}=1 \mathrm{~nm}$. (Maharaj and Bhushan, 2015a). 100

Figure 29: Coefficient of friction data for sliding on $\mathrm{MoS}_{2}$ and $\mathrm{WS}_{2} \mathrm{MWNT}$ at nanotube orientations of $0^{\circ}$ and $90^{\circ}$ (Maharaj and Bhushan, 2015a). Material transfer from the MWNTs to the tip lowers friction compared to sliding on the substrate.... 101

Figure 30: (a) Mechanical properties of thin films with hardness and Young's modulus as a function of contact depth represented by bold square and diamond datum points respectively with corresponding open diamond and square datum points representing bulk mechanical properties. Typical load displacement curves showing (b) creep at maximum loads of 40 and $80 \mu \mathrm{N}$ with a hold period of $200 \mathrm{~s}$, (c) effects of varying strain rate at a maximum load of $80 \mu \mathrm{N}$ for loading-unloading periods of 20 and $200 \mathrm{~s}$ (Maharaj and Bhushan, 2014a).

Figure 31: (a) Typical load displacement indentation curve at a maximum load of $80 \mu \mathrm{N}$ with vertical arrows showing pop-in events and (b) topography maps and 2-D profiles at sections shown by the horizontal arrows before indentation and after indentation. (c) Mechanical properties of Au 500 NPs with hardness and Young's modulus as a function of contact depth represented by bold square and diamond datum points respectively with corresponding open diamond and square datum points representing bulk mechanical properties (Maharaj and Bhushan, 2014a). 109

Figure 32: (a) Typical load displacement compression curve at a maximum load of $80 \mu \mathrm{N}$ for $\mathrm{Au} 500 \mathrm{NPs}$ with vertical arrows showing pop-in events and (b) topography maps and 
2-D profiles at sections shown by the arrows before compression (first row) and after compression (second row) (Maharaj and Bhushan, 2014b)....

Figure 33: Typical load-displacement curve at a maximum load of $50 \mu \mathrm{N}$ with Au $50 \mathrm{NPs}$ and NRs and (b) topography maps and 2-D profiles at sections shown by the horizontal arrows before compression (first row) and after compression (second row). The second row shows topography maps $1 \mathrm{~min}$ after compression and the solid lines and dashed lines show the 2-D profiles after 1 and 4 min respectively (Maharaj and Bhushan, 2014b). . 115

Figure 34: Topography maps and 2-D profiles at sections shown by the horizontal arrows before compression (first row) and after compression (second row) at a maximum load of $200 \mu \mathrm{N}$. The second row shows topography maps $1 \mathrm{~min}$ after compression and the solid lines and dashed lines show the 2-D profiles after 1 and 4 min respectively. Recovery of the nanoparticles $4 \mathrm{~min}$ after compression demonstrates reversed plasticity (Maharaj and Bhushan, 2014b).

Figure 35: Examples of repeat load-displacement curves for Au 50 NPs and NRs (Maharaj and Bhushan, 2014b) and Au 500 NPs (Maharaj and Bhushan, 2014a) with the corresponding maximum loads for each compression event. Evidence of strain hardening can be seen where successive loads results in the displacement being the same or less after unloading. 119

Figure 36: (a) Typical load-displacement curve for low displacement of $50 \mathrm{~nm}$ for $\mathrm{MoS}_{2}$ MWNTs and topography maps and 2-D profiles, at sections indicated by the horizontal arrows before indentation (first row) and after indentation (second row). The dashed square highlights the indent in the "after indentation profile". (b) Typical loaddisplacement curve for high displacement of $100 \mathrm{~nm}$. Vertical arrows point to pop-in events for both curves which occur due breaking of nanotube layers and sudden compression of separated layers. Indentation was performed on separate nanotubes for low and high displacement (Maharaj and Bhushan, 2015b).

Figure 37: Compression load displacement curves for displacements of $50 \mathrm{~nm}, 100 \mathrm{~nm}$ and $300 \mathrm{~nm}$ for $\mathrm{MoS}_{2}$ (Left) and $\mathrm{WS}_{2}$ MWNTs (Right) with the vertical arrows depicting pop-in events. Magnified images are shown inset for displacements of $50 \mathrm{~nm}$ to more clearly illustrate pop-in events which are a result of breaking of individual layers of the nanotubes and sudden compression of separated layers (Maharaj and Bhushan, 2015b).

Figure 38: Compression load displacement curves for displacements of $25 \mathrm{~nm}, 50 \mathrm{~nm}$ and $75 \mathrm{~nm}$ for CNHs with the vertical arrows showing examples of pop-in events. Here popin events are a result from buckling of individual nanohorns (Maharaj and Bhushan, 2015b) 
Figure 39: Examples of repeat load-displacement curves for $\mathrm{MoS}_{2}$ and $\mathrm{WS}_{2} \mathrm{MWNTs}$ at a constant displacement of $50 \mathrm{~nm}$ and CNHs at a constant displacement of $25 \mathrm{~nm}$. Vertical arrows point to pop-in events. Pop-in events for the nanotubes occur due to breaking of walls, and sudden compression of separated layers. For the CNH nanoparticle pop-in events occur due to buckling of individual nanohorns. Evidence hardening can be seen for all three cases where the loads increase at a constant displacement due to compression of individual layers and of MWNTs and nanohorns (Maharaj and Bhushan, 2015b)... 137 


\section{Chapter 1: Introduction}

\subsection{Background}

Nano-objects can be described as objects having at least one dimension that is between 1 and $100 \mathrm{~nm}$. They come in a variety of geometries which include tubes, rods, wires, pillars and spheres. Compared to their bulk material counterparts, many nanoobjects exhibit enhanced mechanical, electrical, magnetic, chemical, friction and wear reducing properties. This makes them attractive for use in many applications on the macro- to nanoscale.

Nano-objects have been investigated for applications that include manipulation and targeting in biomedicine, the oil industry, catalysis and tribology on the macro- to nanoscale.

In biomedicine, nano-objects made of various materials including $\mathrm{Au}$, graphene, iron oxide, polymer and silica have been studied in targeted drug delivery (Duncan, 2003; Ferrari, 2005; Decuzzi et al., 2009; Murakami, 2004; Panyala et al., 2009; Ferrari, 2010; Irvine, 2011). In cancer treatment, for example, nano-objects are either functionalized with biomolecules that recognize and attach to the cancer cells, (Panyala et al., 2009; Ferrari, 2010) or, in the case of iron oxide nanoparticles, directed by an external magnetic 
field (Tran and Webster, 2010). Cancer cells differ from healthy cells due to their reduced intercellular adhesiveness and unregulated growth which allows them to spread throughout the body (Cavallaro and Christofori, 2004; Ackerman and Simon, 2014). The cancer cells are destroyed by either drugs that coat the nanoparticles or by the increasing temperature of the nanoparticles to which cancer cells are susceptible. The cancer cells are destroyed by either drugs that coat the nanoparticles or by the increasing temperature of the nanoparticles to which cancer cells are susceptible. Figure 1a shows a nano-object loaded with a therapeutic drug and functionalized with a biomolecule (ligand), that selectively attaches to receptors in the cancer cell. The drug is then released as the nanoparticle diffuses into the diseased cell resulting in cell death. Several factors need to be considered for the successful use of nano-objects in targeted drug delivery. Biological barriers, including surfaces and the reticulo-endothelial system (RES), which detects and sequesters blood-borne particles, can prevent nano-objects from reaching their intended target (Ferrari, 2010). Smaller nano-objects can diffuse through surfaces and avoid detection by the RES. Nano-objects also come into contact with biological surfaces during diffusion into diseased cells where friction of the nano-objects against the cell wall can reduce the diffusion rate. Reducing agglomeration and friction of nano-objects moving through a liquid and in contact with surfaces is important for efficient transport to diseased sites.

For oil detection applications, as in the example shown in Figure 1b, oxidized carbon black nanoparticles with a polyvinyl alcohol shell are coated with an oil detecting agent, 2,2',5,5'-tetrachlorobiphenyl (PCB). This agent is released on contact with 
hydrocarbons in oil and its absence on recovery and analysis of the nano-objects is an indication of the presence of oil (Berlin, et al., 2011). Agglomeration of nano-objects can prevent flow through porous media, which results in losses and prevents eventual recovery of the nano-objects (Johnson and Elimelech, 1996; Thanikaivelan et al., 2012). Friction on surfaces and during flow in liquids can also hinder recovery. Low friction of the nano-objects over surfaces and within liquids is of interest.

In contaminant removal, magnetic shell cross-linked knedel-like nanoparticles (MSCKs) are of interest. These are composites of polymer layers on iron oxide nanoparticles. In Figure 1c the MSCKs are added to the polluted water (left), where they selectively absorb the oil (center) and are finally removed by introduction of a neodymium magnet (Pavia-Sanders et al., 2013). Reducing agglomeration and fluid friction is important for efficient manipulation of the nano-objects through liquids to contaminated sites.

In applications involving catalysis for hydrogen evolution recovery (HER), nanowires have been used to aid in the catalytic activity as shown in Figure 1d. The nanowires consist of an inner $\mathrm{MoO}_{3}$ core and outer $\mathrm{MoS}_{2}$ shell. The core is used for transporting electrons to edge sites of the outer $\mathrm{MoS}_{2}$ shell, which is catalytically active. This is preferred over bulk $\mathrm{MoS}_{2}$ which has a limited number of edge sites for the same volume of material compared to the nanowires. At these sites the transfer of electrons necessary for recovery of hydrogen from water is enhanced by the $\mathrm{MoS}_{2}$, which improves the efficiency of the HER (Chen et al., 2011). Low friction of water moving over the nanowires is important for efficient flow over edge sites and conversion to hydrogen. 
Figure 1: Examples of nano-objects in applications in (a) drug delivery, where nanoobjects coated with a drug, targets cancer cells and release their therapeutic payload resulting in death of the cancer cell (Tran and Webster, 2010), (b) oil detection, where carbon black nano-objects are coated with an oil detecting agent. After injection into the ground, the agent is released on contact with hydrocarbons and this is used as an indication of the presence of oil on recovery and analysis of the nanoparticle (Berlin et al., 2011), (c) contaminant removal with magnetic cross-linked knedel-like nanoparticles (MSCKs). The MSCKs are added to the polluted water (left), where they selectively absorb the oil (center) and are finally removed by introduction of a neodymium magnet (Pavia-Sanders et al., 2013) and (d) catalysis for hydrogen evolution recovery (HER), using nanowires. The nanowires consist of an inner $\mathrm{MoO}_{3}$ core and outer $\mathrm{MoS}_{2}$ shell. The core is used for transporting electrons to edge sites of the inner $\mathrm{MoS}_{2}$ shell which is catalytically active. At these sites the transfer of electrons necessary for recovery of hydrogen from water is enhanced by the $\mathrm{MoS}_{2}$, which improves the efficiency of the HER (Chen et al., 2011). 
Examples of nano-objects used in various applications

Nano-objects selectively targeting tumor cells for drug delivery

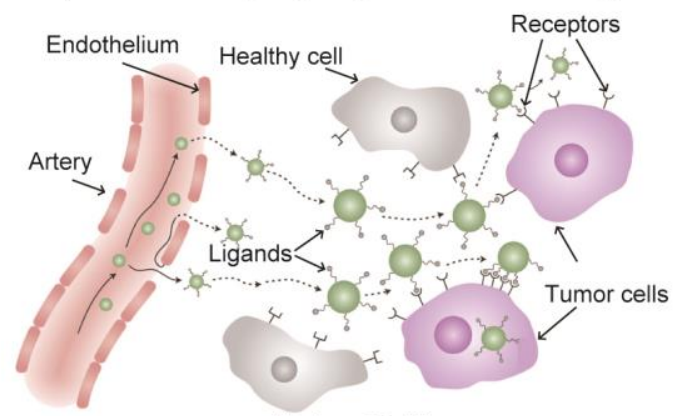

(Irvine, 2011)

(a)

Nano-objects being used for oil detection



(Berlin et al., 2011)

(b)
Nano-objects removing pollutants from water



Removal of loaded MSCKs via magnetic force


Sequestration of pollutants by MSCKs

(Adapted from Pavia-Sanders et al., 2013)

(c)

Nanowires being used for hydrogen evolution recovery (HER)

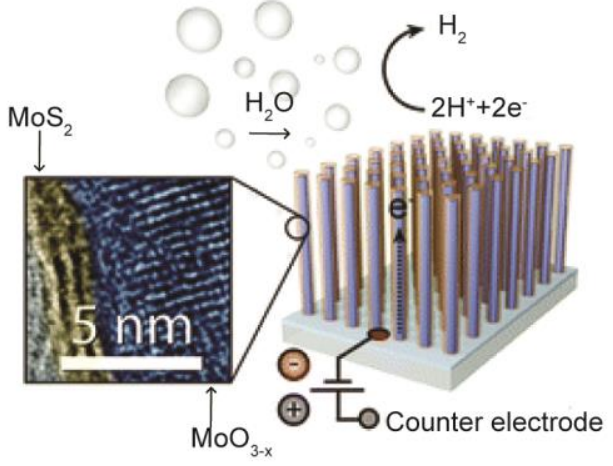

(Adapted from Chen et al., 2011)

(d)

Figure 1 
Nano-objects have demonstrated the ability to reduce friction and wear in applications on the macroscale. For applications on the micro/nanoscale, increasing the lifetime and efficiency of individual components of systems is crucial to the commercialization of micro/nanosystems. Adhesive and friction forces can hinder device operation and reliability (Bhushan, 2011). Several studies on the nanoscale have demonstrated their ability to reduce friction and wear in both dry and submerged in liquid environments. This has been attributed their lower real area of contact and the studies are discussed in the section on nano-object studies. In these environments nano-objects can be deformed locally or the entire nano-object can be compressed. Knowledge of the mechanical behavior during loading and unloading in these environments is essential for sustained use.

\subsection{Studies of nano-objects}

In many applications described, nano-objects come into sliding contact with each other and the surfaces in which they are used. The nano-objects can be deformed locally, where they may be indented, or globally, where the entire nano-object is compressed. During sliding contact, asperities present on the mating surfaces come into contact and deform to support the load. Adhesive contacts are formed with the asperities by either physical or chemical interactions. When the surfaces move relative to each other a shear force is needed to break the adhesive bonds formed at the real area of contact. The friction measured is given as:

$$
F=A_{r} \tau
$$


where $A_{r}$ is the real area of contact and $\tau$ is the average shear strength of the contacts. Both reduction in shear strength and contact area reduces friction as well as wear. Studies have been performed to understand interfacial friction and wear, their mechanical properties and deformation mechanisms.

Nano-object contact studies have been performed in dry and submerged in liquid environments using an atomic force microscope (AFM). Single-nano-object contact studies provide understanding of friction mechanisms of nano-objects sliding on surfaces. Multiple nano-object contact studies investigate the ability of several nano-objects to reduce friction and wear between sliding surfaces. Further studies on friction mechanisms have also been done on a single nano-object with an AFM where the effects of topography, orientation, and scale on friction were examined. Studies have also been done on local deformation (indentation) and global deformation (compression) with a sharp tip and flat punch respectively, to understand mechanical behavior. Local deformation studies allow for investigation of scale effects on hardness and Young's modulus. Global deformation studies investigate occurrence of reverse plasticity and resistance to deformation due to repeat loading.

\subsubsection{Single nano-object contact studies}

In single nano-object-contact studies, nano-objects are moved with AFM probes where they can be manipulated by either being pushed laterally or the probe is placed on top of the nano-object and translated. Nano-objects used for these studies include antimony (Sb) NPs, Au NPs and NRs, CNHs, latex NPs, silica dioxide $\left(\mathrm{SiO}_{2}\right) \mathrm{NPs}, \mathrm{MoS}_{2}$ 
and $\mathrm{WS}_{2}$ MWNTs and carbon nanotubes (CNTs). Studies have been performed in dry and submerged in liquid environments.

Single nano-object contact studies in dry environments, where the nano-objects are pushed laterally show that there is a friction force dependence on contact area (Ritter et al., 2002, 2005; Dietzel et al., 2007; Mougin et al., 2008; Palacio and Bhushan, 2008; Hui and Regnier, 2012; Maharaj and Bhushan, 2012; Maharaj et al., 2013) and relative humidity (Mougin et al., 2008; Palacio and Bhushan, 2008). These pioneering studies have been conducted in both continuous and intermittent contact modes. Lower friction forces occur due to reduced contact area of nano-objects and lower work of adhesion (Maharaj et al., 2013).

Nano-objects have also been manipulated with AFM probes attached to a nanomanipulator (Lahouij et al., 2011; Tevet et al., 2011). In this case the truncated tip of the probe, which can have diameters from $350 \mathrm{~nm}$ to $500 \mathrm{~nm}$, is placed on top of the nano-object and translated. By applying a normal load to an inorganic fullerene (IF) $\mathrm{MoS}_{2}$ nano-object, Lahouji et al. (2011) suggested rolling, sliding and exfoliation of the nano-object as the AFM probe was translated over it in dry environments. These experiments were performed inside of a transmission electron microscopy (TEM) chamber which allowed for real time observation of the mechanisms. Tevet et al. (2011) also suggested rolling, sliding and exfoliation of IF-WS 2 and $\mathrm{MoS}_{2}$ nanoparticles inside of a high resolution scanning electron microscopy (HRSEM) chamber. In this study a normal load was also applied on top of the nanoparticles and translated in dry environments. 
Single nano-object contact studies by laterally pushing nano-objects have also been performed under liquid environments. Resch et al. (2000) demonstrated that Au nanoparticles can be pushed in water and ethanol in intermittent contact mode similar to pushing in air. Maharaj and Bhushan (2012) and Maharaj et al. (2013) have also pushed Au NPs and NRs, $\mathrm{MoS}_{2}$ and $\mathrm{WS}_{2}$ MWNTs and CNHs submerged in liquid environments and determined the friction forces of the nano-objects during pushing. It was shown that in submerged in liquid environments, that the friction forces were lower compared to dry environments due to elimination of meniscus forces and the presence of a low shear strength surface for sliding. Friction forces were influenced by real area of contact, roughness and work of adhesion of the nano-objects.

\subsubsection{Multiple nano-object contact studies}

Nanoparticles including Au, antimony (Sb), $\mathrm{C}_{60}, \mathrm{CNHs}, \mathrm{MoS}_{2}, \mathrm{WS}_{2}, \mathrm{Fe}_{2} \mathrm{O}_{3}$ and $\mathrm{SiO}_{2}$; nanorods including zinc sulfide $(\mathrm{ZnS})$, zinc oxide $(\mathrm{ZnO}), \mathrm{Au}$ and silver vanadium oxide $\left(\mathrm{AgVO}_{3}\right)$; and nanotubes including $\mathrm{MoS}_{2}, \mathrm{WS}_{2}$, and $\mathrm{CNTs}$ have been studied in friction and wear reduction on the macro- to nanoscale. Studies were carried out in dry environments on the macroscale (Bhushan and Gupta, 1991; Rapoport et al., 2001;

Tanaka et al., 2005; Maharaj and Bhushan, 2012, 2013, Maharaj et al., 2013, 2014a), microscale using a surface force apparatus (SFA) (Singh et al., 2010, Zhang and Zhang, 2012) and nanoscale using an AFM (Luthi et al., 1994; Ritter et al., 2005; Sitti 2004; Dietzel et al., 2007; Mougin et al., 2008; Palacio and Bhushan, 2008; Lahouij et al., 2011; Tevet et al., 2011; Hui and Regnier, 2012, Maharaj and Bhushan, 2012, 2013; Maharaj et 
al., 2013, Maharaj and Bhushan, 2014a). Studies have also been carried out in submerged in liquid environments on the macroscale (Bhushan and Gupta, 1991; Hu et al., 1998;

Cizaire et al., 2002; Greenberg et al., 2004; Joly-Pottuz et al., 2005; Rapoport et al., 2005; St. Dennis et al., 2011; Kalin et al., 2012, Maharaj and Bhushan, 2012, 2013; Maharaj et al., 2013, Maharaj and Bhushan, 2014b), microscale with an SFA (Gourdon et al., 2004; Akbulut et al., 2006) and nanoscale with an AFM (Resch et al., 2000; Maharaj and Bhushan, 2012, 2013; Maharaj et al., 2013; Maharaj and Bhushan, 2014b).

In these studies, reductions in friction and wear are as a result of mechanisms which include reduced real area of contact, rolling and sliding. Exfoliation and tribofilm formation also results in lower friction for nano-objects made of materials such as $\mathrm{MoS}_{2}$ and $\mathrm{WS}_{2}$. These materials have a lamellar structure in which there are strong covalent bonds between atoms of the same layer and weak van der Waals forces between the layers as seen in Figure 2a. During sliding, the weakly bonded layers shear past each other, an example of which is shown in Figure $2 b$. This results in reduced friction and wear (Bhushan and Gupta, 1991; Bhushan, 2013). 


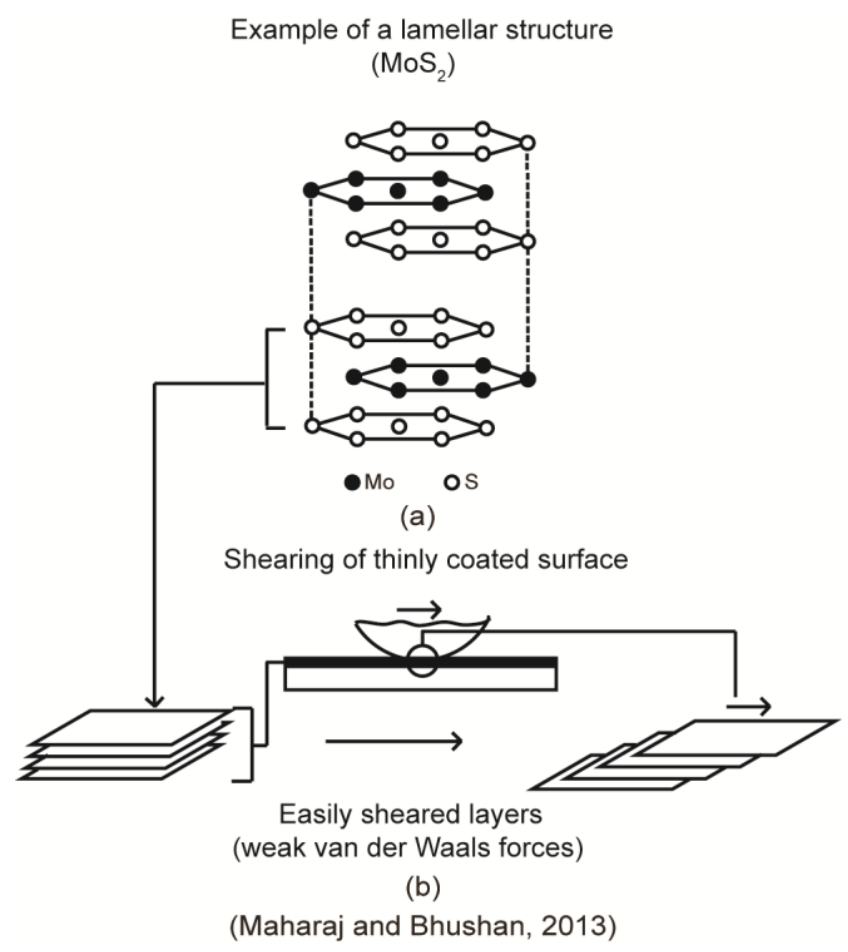

Figure 2: Friction reduction occurs as a result of weak van der Waals forces between layers which allows for easy shearing of the layers (Maharaj and Bhushan, 2013a).

On the macro- to nanoscale, lower coefficient of friction is observed in liquids compared to air when sliding on nano-objects due to the presence of a low shear strength film. In multiple nano-object contact studies, coefficient of friction was also lower in liquids due to elimination of liquid mediated contact as shown in studies by Maharaj and Bhushan (2012, 2013), Maharaj et al. (2013), Maharaj and Bhushan (2014b). In liquid mediated contact, adhesive meniscus forces occur due to the presence of water vapor in 
the atmosphere. The water vapor condenses around the contact area of the nano-objects and surface on which they rest. The coefficient of friction is given as:

$$
\mu=\frac{F}{W}=\mu_{r}\left(1+\frac{F_{m}}{W}\right)+\frac{F_{v \| \mid}}{W}
$$

where $\mu_{r}$ is the true coefficient of friction in the absence of menisci and $W$ and $F_{m}$ are the applied normal load and meniscus force in the normal direction. $F_{v \| \mid}$ is the viscous force in the sliding direction (Bhushan, 2013). Friction force $\left(\mu_{r} / W\right)$ is dependent on material properties and surface topography. $F_{m}$ is dependent on roughness parameters, liquid type and film thickness. In equation 2 , the $\mu_{r} F_{m}$ and $F_{v \|}$ terms comprise the friction force due to liquid-mediated adhesion. These terms are eliminated when submerged in liquid environments resulting in lower friction compared to air.

\subsubsection{Friction on a single nano-object}

To understand the frictional behavior on nano-objects, AFM studies on a single nanoobject such as $\mathrm{MoS}_{2}$ and WS 2 multi-walled MWNTs have been carried out. These studies provide useful information on scale effects and local changes in friction due to topographic variations and material effects related to surface chemistry and nano-object orientation (Ruan and Bhushan, 1994; Bhushan et al., 2008; Bhushan, 2011; Jelenc and Remskar, 2012; Maharaj and Bhushan, 2015a). In these studies, scale effects of friction were observed where lower coefficients of friction were found sliding on the nanoobjects compared to bulk. 


\subsubsection{Mechanical behavior of nano-objects and thin films}

Research has shown that mechanical properties on the micro/nanoscale are different from bulk and are scale dependent as observed in studies of bulk solid surfaces, surface thin films and micro- to nano-objects. Mechanical properties can either be reduced or similar to bulk in some cases or enhanced as material dimensions go from the macro- to nanoscale.

Reduced or similar mechanical properties have been observed in some studies of micro/nano-objects where decreasing diameters results in reduced or similar yield strength and hardness compared to bulk. In the case of reduced properties, this is due to the inverse Hall-Petch effect (Schaefer et al., 1993; Chen et al., 2009; Jang and Greer, 2011) where, mechanical properties below a critical grain size decreases with grain size. This occurs as the grain is too small to support enough dislocations to resist deformation. Deformation now occurs through grain rotation and grain boundary sliding. Various material defects, such as existing cracks and spaces within nano-objects made of layered material, can also prevent observation of enhanced mechanical properties (Maharaj and Bhushan 2015b).

Of particular interest are cases where properties are enhanced leading to the 'smaller is stronger' phenomenon. An overview of several of these studies, in which mechanical properties improve as scale is reduced, is presented in Table A.1 (see Appendix A). Also presented are material dimensions and associated theories. 
In studies of bulk solid surfaces (Gane and Cox, 1970; Pethica et al.,1983, Pharr and Oliver ,1989; Bhushan and Koinkar,1994; Bhushan et al. ,1996; Mante et al.,1999; Grillo et al., 2003) and thin films (Doerner et al., 1986; Cao et al., 2006; Dietiker et al., 2008; Knapp and Follstaedt, 2004; Maharaj and Bhushan, 2014a) made of various materials including $\mathrm{Al}, \mathrm{Au}, \mathrm{Ag}, \mathrm{Cu}, \mathrm{GaAs}, \mathrm{GaP}, \mathrm{Si}, \mathrm{Ti}, \mathrm{ZnSe}$ and $\mathrm{Ni}$, scale effects on hardness with respect to depth of penetration or indentation size effect (ISE) and decreasing film thickness have been reported. In the ISE, studied by several groups (Ashby, 1970; Fleck and Hutchinson, 1993; Nix and Gao, 1998), for example, Pharr and Oliver (1989) and Bhushan et al. (1996), found that decreasing indentation depth resulted in higher hardness of $\mathrm{Ag}$ and $\mathrm{Si}$ surfaces, respectively. In both cases the nanohardness was higher than that of bulk hardness due to dislocations generated as a result of large strain gradients. Other studies revealed the relationship between film thickness and hardness. For example, Cao et al. (2006) and Dietiker et al. (2008), demonstrated that as film thickness decreased for $\mathrm{Ag}$ and $\mathrm{Au}(10-2000 \mathrm{~nm})$ and $\mathrm{Au}(31-858 \mathrm{~nm})$ respectively, hardness increased. Hardness dependence on film thickness can be explained by either the Hall-Petch effect (Hall, 1951; Petch, 1953; Li, 1969) or dislocation constraint, where the hard substrate limits the movement of dislocations.

Scale dependence is also seen in studies of various micro/nano-objects. Enhanced mechanical properties were observed in nano-objects (Thilly et al., 2002; Greer et al., 2005; Wu et al., 2005; Shan et al., 2008; Dieteker et al., 2011, Mordehai et al., 2011a; Maharaj and Bhushan, 2014a) made of various materials including $\mathrm{Au}, \mathrm{Cu}, \mathrm{Nb}$ and $\mathrm{Ni}$ where decreasing diameters results in an increase in micro/nano-object yield stress and 
hardness. Indentation tests of $\mathrm{Cu} / \mathrm{Nb}$ microwires $(1-10 \mu \mathrm{m})$ by Thilly et al. (2002) showed that lower diameter resulted in higher yield stress following the 'smaller is stronger' phenomenon. In compression studies involving $\mathrm{Au}(0.4-7.5 \mu \mathrm{m})$ micropillars a similar observation was made where higher yield strengths were observed compared to bulk with decreasing micropillar diameter (Greer et al., 2005).

The increase in yield strength or hardness seen with nano-objects has been explained by the dislocation starvation model for single crystalline nano-objects or the Hall-Petch effect for polycrystalline nano-objects. In the dislocation starvation model, the absence of dislocations in the interior of the nano-object does not allow for plastic deformation to occur. Similar to thin films, for indentation of micro and nano-objects, there is also a contribution to hardening due to the occurrence of large strain gradients at shallow depths for both single and polycrystalline materials.

\subsection{Choice of nano-objects and liquids for this study}

$\mathrm{Au}$ NPs and NRS, $\mathrm{MoS}_{2}$ and $\mathrm{WS}_{2}$ MWNTs and CNHs are selected for methodology and trends to guide in selecting nano-objects for various applications.

Au nano-objects have been used in applications requiring targeting in biomedicine due to their chemical inertness and low to no toxic effects. In tribological studies, its small contact area and mobility due to its spherical shape allows it to roll and slide have resulted in reduced friction and wear on the macro- to nanoscale in dry and submerged in liquid environments (Bhushan and Gupta, 1991; Mougin et al., 2008; Palacio and Bhushan, 2008). 
$\mathrm{MoS}_{2}$ and $\mathrm{WS}_{2} \mathrm{MWNTs}$ have also been studied in tribology on the macro- to nanoscale in dry and liquid environments and increasingly, in other applications for catalysis in HER. MoS 2 and $\mathrm{WS}_{2}$ MWNTs have been shown to reduce friction and wear similar to Au nano-objects. In addition, the exfoliation of the outer layers of the nanotubes and subsequent formation of an easily shearable film aids in friction and wear reduction (Bhushan and Gupta, 1991; Cizaire et al., 2002; Joly-Puttz et al., 2005; Rapoport et al., 2005; Lahouji et al., 2011; Tevet et al., 2011; Kalin et al., 2012).

CNHs have been the subject of myriad of studies due to their unique morphology and wide ranging properties of graphene, which includes chemical stability, low surface energy (Kim et al., 2011) and high thermal (Sani et al., 2010) and electrical conductivity (Geim et al., 2007). Several studies have made use of CNHs for various applications including storage of fuel gases (Murata et al., 2004), use as a potential drug delivery system in cancer treatment (Murakami et al., 2004) and tribology on macro- to nanoscale (Tanaka, et al., 2005). Tribological studies have shown the additional roughness provided by the individual nanohorns can further reduce the contact area of the nanoparticle. This, together with the low meniscus force contribution to adhesion, further enhances the ability to reduce friction and wear.

For submerged in liquid environments, water, dodecane and glycerol were used. They have been utilized in friction and wear studies as they are simple liquids without additives and their properties are well known. The low viscosity of water and its ability to provide a surface of low shear strength (Bhushan and Sundararajan, 1998) for sliding, makes it a good candidate as an operating fluid. For glycerol, studies have been 
performed on the macroscale (Nalam et al., 2010) and Dodecane used with $\mathrm{ZnS}$ nanorod additives (Akbulut et al., 2006) have resulted in reduced friction and wear on the micro/nanoscale. For the nano-objects studied, knowledge of interfacial friction and wear, their mechanical properties and deformation mechanisms are of general interest.

\subsection{Objective and layout}

In this thesis, results of studies performed in friction, wear and mechanical behavior will be presented in relation to Au NPs and NRs, $\mathrm{MoS}_{2}$ and $\mathrm{WS}_{2}$ MWNTs and CNHs. The studies discussed are shown in Figure 3 and are single and multiple nanoobject contact in dry and liquid environments, friction on single nano-objects and mechanical behavior of nano-objects. In single nano-object contact, Au NPs and NRs, $\mathrm{MoS}_{2}$ and $\mathrm{WS}_{2}$ MWNTs and CNHs have been studied with an AFM where they are manipulated by lateral pushing. Friction forces were examined in dry and compared to submerged in liquid environments for the first time. Friction was found to be dependent on size, shape, material and roughness. Friction was also lower in submerged in liquid environments compared to dry conditions. These studies provide an understanding of the nature of the friction mechanism of nano-objects at the initiation of sliding.

In multiple nano-object contact studies, the effect of nano-objects on friction and wear reduction was determined. Sliding takes place over several nano-objects. Real area of contact, rolling, sliding and exfoliation and tribofilm formation were studied for their effect on friction and wear. Results of studies on the macroscale were compared to the nanoscale to understand scale effects of friction. 
Figure 3: Illustration of the studies in friction, wear and mechanical behavior of nanoobjects. The studies examined were single and multiple nano-object contact in dry and liquid environments, friction on single nano-objects and mechanical behavior of nano-objects during indentation and compression (Left column). Observations for each study are given in the column on the right. 
Studies performed for understsnding of friction, wear and mechanical behavior of nano-objects Studies Observations



Figure 3 
To further understand the friction mechanism, AFM studies on $\mathrm{MoS}_{2}$ and $\mathrm{WS}_{2}$ MWNTs have been performed where sliding takes place on a single nano-object. These studies examine the effects of topography, scale and orientation on friction. Several mechanisms are used to explain results of the studies.

Mechanical behavior studies have also been performed on Au NPs, NRs and thin film, $\mathrm{MoS}_{2}$ and $\mathrm{WS}_{2} \mathrm{MWNTs}$ and CNHs. Nanoscale measurements were made to study scale effects. By using a sharp tip and a flat punch local deformation (indentation) and global deformation (compression) behavior were studied. Repeat compression tests of all nano-objects were performed to investigate nanoscale hardening. Results are discussed and explained in terms of dislocation driven mechanisms and theories.

The results of the studies are discussed and various mechanisms are used to explain the friction and wear behavior of nano-objects and mechanical behavior when subjected to various loading and unloading conditions. The nano-objects selected will aid in determining methodology and trends, which guide in the selection of nano-objects for various applications. 


\section{Chapter 2: Mechanisms}

\subsection{Introduction}

In this section, mechanisms for observed mechanical properties of materials on the nanoscale are described. Explanations of the various mechanisms are important to aid in discussing and understanding the current research.

Plastic deformation is driven by the presence or absence of sets of atoms that disrupt the regular atomic arrangements in the lattice planes, called dislocations.

For a polycrystalline material as an example, Figure 4 shows dislocations sources in the grain. Dislocations originate from the grain boundary, grain interior, through multiplication of existing dislocations during loading and from geometrically necessary dislocations generated to accommodate strain gradient in nanoindentation at low penetration depth.

After initial slip, as more dislocations are generated and the dislocation density increases less slip occurs. This leads to an increase in yield stress or hardness as the dislocations entangle with each other and or are stopped at grain boundaries in a material. They help to explain certain observable effects such as the indentation size effect (ISE) and Hall-Petch effect. In the case of the ISE, contributions to enhanced hardness can occur in either single or polycrystalline nano-objects. Other mechanisms of interest 
involve the creation of stacking faults and the types of dislocations associated with them. The mechanisms of each are given in the following sections.

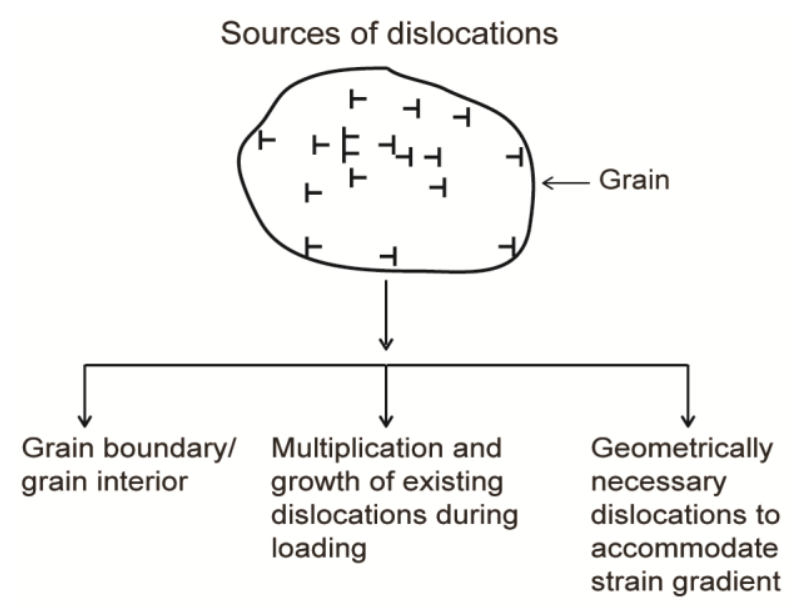

(Maharaj and Bhushan, 2014a)

Figure 4: Flowchart illustrating dislocation sources, in a grain, from the grain boundary and grain interior, through multiplication and growth of an existing dislocations and from geometrically necessary dislocations needed to accommodate strain gradient (Maharaj and Bhushan, 2014a).

\subsection{Indentation size effect-Strain gradient plasticity}

In a material before indentation of the surface, dislocations exists called statistically stored dislocations (SSDs). As the material surface is indented, dislocations called geometrically necessary dislocations (GNDs) are created. The GNDs allow for plastic deformation of the material beneath the indenter, as depicted in Figure 5. At shallower indentation depths larger strain gradients lead to a greater density of GNDs. 




Figure 5: Illustration of the strain gradient plasticity theory where high strain gradients occur at shallow indentation depths with a sharp tip.

This leads to geometrically necessary dislocations (GNDs) being generated to accommodate the strain gradient and subsequent permanent deformation. The GNDs entangle and impede further movement of existing dislocations as well as the formation and movement of new dislocations (Based on Nix and Gao, 1998). The increase with GNDs along with SSDs, hinder the formation and movement of new dislocations and results in a strengthening effect (Ashby, 1970; Fleck and Hutchinson, 1993; Nix and Gao, 1998). This accounts for the observed increase in hardness at shallower indentation depths. This phenomenon was first modeled by Nix and Gao (1998) according to the following relation

$$
\mathrm{H} / \mathrm{H}_{0}=\left(1+\mathrm{h}^{*} / \mathrm{h}\right)^{1 / 2}
$$

where $\mathrm{H}$ is the hardness at a given indentation depth of $\mathrm{h}$, with $\mathrm{H}_{0}$ being the hardness at a large indentation depth and $\mathrm{h}^{*}$ is a characteristic length which is dependent on indenter shape, shear modulus and $\mathrm{H}_{0}$. The ISE contributes to increased hardness in bulk solid surfaces, thin films and nano-objects. 


\subsection{Hall-Petch effect}

Generation of dislocations leads to the Hall-Petch effect through the dislocation pile up mechanism or the dislocation density mechanism in polycrystalline materials and are described below. In both mechanisms as the grain size is reduced, the yield stress increases resulting in higher hardness in the case of indentation as stated previously (Hall, 1951; Petch, 1953; Li, 1969; Abson and Jonas, 1970). It should be noted that the strengthening effect can be as a result of a combination of mechanisms.

\subsubsection{Dislocation pile up mechanism}

As the nanoscale is approached, polycrystalline thin films and nano-objects by virtue of their physical dimensions will be composed of materials that have a nanocrystalline structure i.e. nanometer size grains; as compared to the coarse grained materials observed on the macroscale with bulk material, where grain sizes can vary from 10-300 $\mu \mathrm{m}$ (Meyers et al., 2006). In the pile up mechanism illustrated in Figure 6a, for a given applied stress $\tau$, on the grains illustrated by the vertical arrows, dislocations are generated along slip lines, as depicted by the dashed lines, and eventually pile up against the grain boundary. The stress at the grain boundary is called the pile up stress, given as

$$
\tau_{\mathrm{p}}=\mathrm{n}\left(\tau-\tau_{\mathrm{i}}\right)
$$


Figure 6: Illustration of (a) Hall-Petch (Based on Hall, 1951; Petch, 1953) effect by dislocation pile up mechanism (Based on Li, 1969; Jonas, 1970), where dislocations pile up against the grain boundary under an applied stress $\tau$. The stress at the boundary $\tau_{\mathrm{p}}$ (pile up stress) which is a function of $\tau$ and $\tau_{\mathrm{i}}$ (internal stress) of the dislocations is larger for a bigger grain size (A) on the macroscale due to the larger number of dislocations compared to that for a smaller grain size (C) on the nanoscale. The number of dislocations (n) within the grain on the nanoscale is smaller compared to the macroscale, requiring a larger $\tau$ to create a pile up stress ( $\left.\tau_{\text {critical }}\right)$ high enough for dislocation slip and plastic deformation which results in a higher yield stress and (b) Dislocation density mechanism where there is a larger overall grain boundary area as grains become smaller within the same volume, resulting in a greater density of dislocations which impede the formation and motion of new dislocations to accommodate strain gradients and results in higher yield stress (Based on Ashby, 1970). 
Illustration of mechanisms for Hall-Petch effect

Increase in yield stress with a decrease in grain size

(Based on Hall, 1951; Petch, 1953)

Dislocation pile up mechanism

(Basd on Li,1969; Abson and Jonas, 1970)



On the nanoscale, higher $\tau$ required to generate large enough

$\tau_{p}$ for dislocation slip which leads to increased yield stress

Dislocation density $(\rho)$ mechanism

(Based on Ashby, 1970)
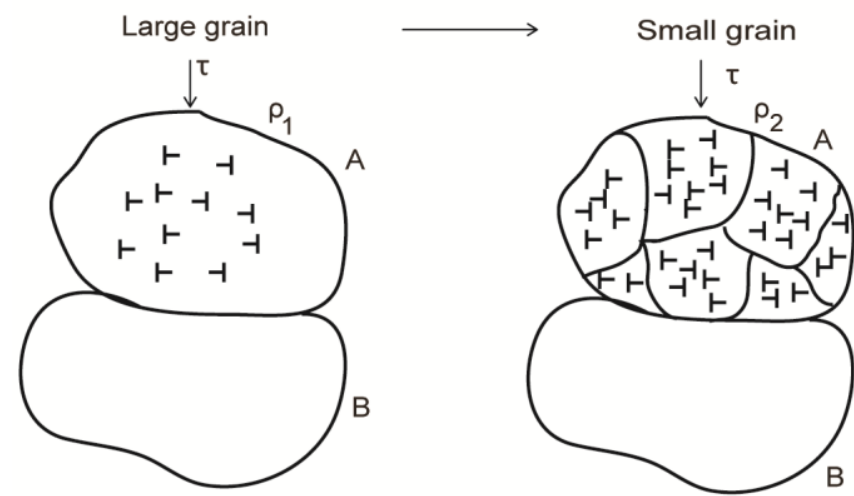

On the nanoscale, larger overall grain boundary area within the same volume results in greater $\rho$ which impedes formation and movement of new dislocations resulting in increased yield stress

(b)

Figure 6 
where $\tau_{\mathrm{i}}$ is the friction stress opposing the movement of the dislocations. The number of dislocations in the pile up in grain $\mathrm{A}\left(\mathrm{n}_{1 \mathrm{~A}}\right)$ is greater than in grain $\mathrm{C}\left(\mathrm{n}_{2} \mathrm{C}\right)$ due to the larger size which leads to a greater $\tau_{\mathrm{p}}$. For slip to occur across the grain boundary, $\tau_{\mathrm{p}}$ must be greater than the critical stress, $\tau_{\text {critical. }}$ A higher initial $\tau$ is therefore required on grain $\mathrm{C}$ before the critical stress is reached to allow slip to occur and plastic deformation to continue, which results in a higher yield stress compared to grain A (Hall, 1951; Petch, 1953; Li, 1969; Abson and Jonas, 1970).

\subsubsection{Dislocation density mechanism}

As mentioned earlier, dislocations can be generated from different sources as shown in Figure 2.1. As the grain size becomes smaller shown in Figure 6b, there is a larger overall grain boundary area within the same volume on the nanoscale (right) compared to the macroscale (left), resulting in a greater number of dislocations per unit area or density $(\rho)$. This entanglement of dislocations impedes the formation and motion of new dislocations and multiplication of existing dislocations necessary to accommodate strain gradient and subsequent deformation. This results in greater resistance to deformation and increased yield stress (Ashby, 1970; Gracio, 1994; Conrad and Jung, 2005).

\subsection{Stacking faults}

It is known that movement of dislocations by slip allows for plastic deformation to occur. In lamellar structures as found in $\mathrm{MoS}_{2}$ and $\mathrm{WS}_{2}$, stacking faults that are created 
during material formation are accompanied by partial dislocations. Local regions in a crystal consist of layers of atoms on top of each other in a repeating pattern known as the stacking order. The first layer is designated as the 'A' layer and successive layers are given a new letter designation such as ' $\mathrm{B}$ ' or ' $\mathrm{C}$ ' if offset to the original layer and each other. Stacking faults results when this order is interrupted by either introduction or removal of an extra plane of atoms as shown in Figure 7 (Hull and Bacon, 2001). Where the fault ends within the crystal, the area separating the faulted region from the perfect crystal is a partial dislocation. Partial dislocations can either prevent or promote slip and plastic deformation. Figure 7 illustrates the concept of stacking faults and partial dislocations with Frank and Shockley partials.

Frank partials are sessile and do not move by dislocation slip and prevents plastic deformation by obstructing movement of dislocations that can slide. Figure 7a shows an intrinsic case, as an example, where a plane of atoms are missing within a layer of atoms with 'A to A' arrangement. The plane of atoms above and below, shift and settle into the vacant area. It is energetically unstable to have two layers of atoms with similar arrangement stacked on top of each other. To return to a more stable state, the internal arrangement of atoms above the missing layer changes from a ' $\mathrm{B}$ to $\mathrm{B}$ ' to a ' $\mathrm{B}$ to $\mathrm{C}$ ' configuration. This causes subsequent internal layers above it, to also rearrange themselves to accommodate this change. Figure $7 \mathrm{~b}$ shows a stacking fault resulting from a Shockley partial as an example. This results in a stacking fault where a ' $\mathrm{C}$ ' arrangement of atoms is found within an ' $\mathrm{A}$ ' layer shown within the dashed lines. In this case the ' $\mathrm{C}$ ' arrangement is preferred to one with ' $\mathrm{B}$ ' since it is more energetically favorable. 
Shockley partials are glissile and move leading to slip and plastic deformation (Hull and Bacon, 2001).

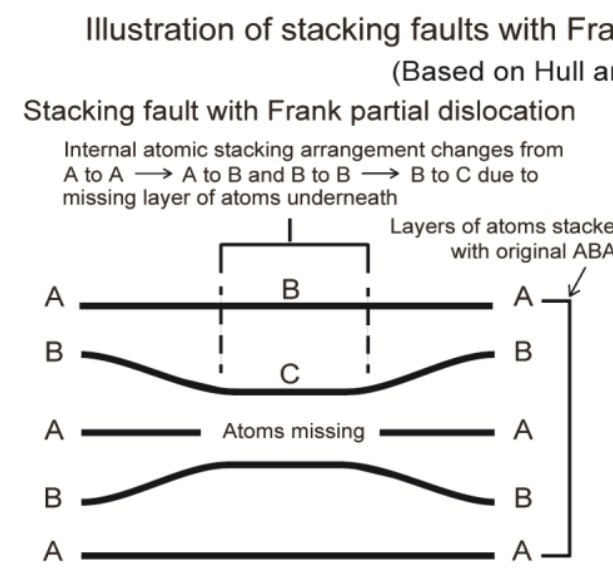

(a)

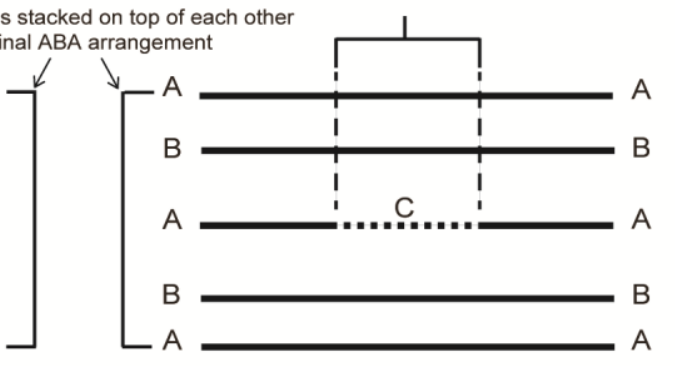

(b)

Figure 7: Schematic showing (a) stacking faults resulting from the removal of a plane of atoms with sessile Frank partials dislocations in the region between the faulted and perfect crystal. The layer directly above the missing atoms changes internally from a ' $\mathrm{B}$ to $\mathrm{B}$ ' to ' $\mathrm{B}$ to $\mathrm{C}$ ' arrangement to prevent an energetically unstable arrangement. The subsequent layer changes from an 'A to A' to 'A to B' arrangement, and (b) stacking faults with glissile Shockley partials in the region between the faulted and perfect crystal. These faults are as a result of an insertion of a plane of atoms with ' $\mathrm{C}$ ' arrangement between a layer with an 'A' arrangement. The ' $\mathrm{C}$ ' arrangement is more energetically favorable to a 'B'arrangement (Based on Hull and Bacon, 2001). 


\section{Chapter 3: Experimental}

In this section Au thin film, NPS and NRs, $\mathrm{MoS}_{2}$ and $\mathrm{WS}_{2} \mathrm{MWNTs}$ and $\mathrm{CNHs}$ used in the studies are described along with their method of preparation. This is followed by descriptions of the experiments and the measurements taken.

\subsection{Materials and sample preparation}

$\mathrm{Si}$ (100) silicon wafers with a native oxide layer (University Wafers, Boston, MA) were ultrasonically cleaned in DI water, followed by isopropyl alcohol (IPA) and finally acetone for 15 min each. Nano-objects were deposited on these substrates to carry out various experiments.

Several examples of nano-objects used are shown in the micrographs and described. Examples of Au nano-objects are shown (Nanopartz, Inc., Loveland, CO) in Figure 8. In the transmission electron microscopy (TEM) images, the Au NPs and NRs are $50 \mathrm{~nm}$ in diameter with a nanorod length of $200 \mathrm{~nm}$ (Nanopartz.com, 2015). Figure 9 shows Au NPs $\sim 500 \mathrm{~nm}$ in diameter (Alfa Aesar, Ward Hill, MA) and a polycrystalline Au film of $100 \mathrm{~nm}$. Figure 9 shows the Au film on a silicon substrate (left) with a magnified view of the section highlighted by the dashed lines shown on the right. In the magnified view the dashed lines highlight the 
grains. Figure $9 \mathrm{~b}$ shows several Au nanoparticles (left) with a magnified view of a single nanoparticle highlighted by the dashed lines. The view on the right shows grains highlighted by the dashed lines. To observe the grains within the Au film and

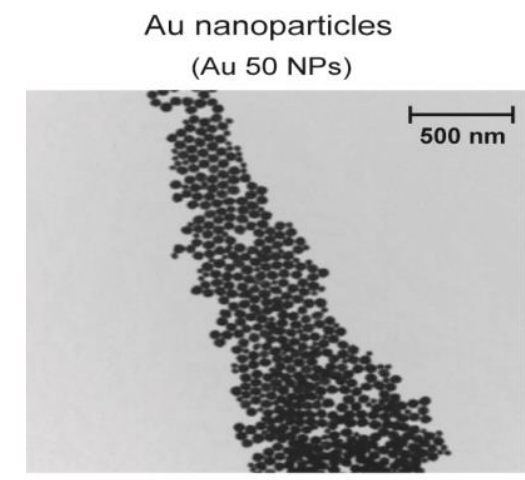

(a)

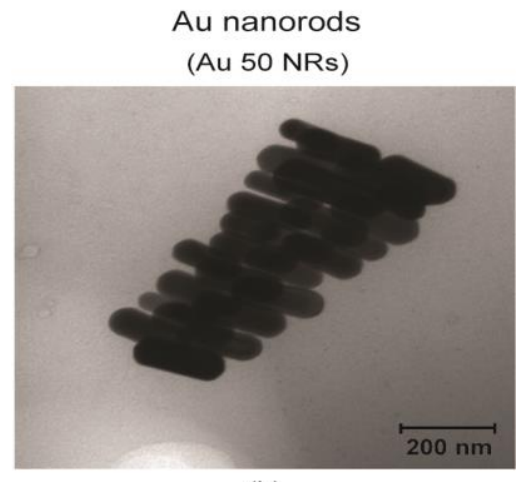

(b)

(Nanopartz.com, 2015)

Figure 8: (a) TEM images of (a) spherical Au nanoparticles approximately $50 \mathrm{~nm}$ in diameter (b) Au nanorods $50 \mathrm{~nm}$ in diameter and $200 \mathrm{~nm}$ in length (Nanopartz.com, 2015). 
nanoparticles, focused ion beam (FIB) milling and transmission electron microscopy (TEM) were employed. Cross-sections of samples were cut out by FIB milling (Nova NanoLab 600, FEI, Hillsboro, OR) using a $\mathrm{Ga}^{+}$ion beam accelerated at a voltage of 30 $\mathrm{kV}$ with currents ranging from $0.03 \mathrm{nA}$ to $28 \mathrm{nA}$. A Pt coating was deposited on both sets of samples to protect the surfaces during milling. The cross-sections were then lifted out using a micro manipulator and placed in a holder and observed using a TEM system (Tecnai F20, FEI, Hillsboro, OR) operated at a voltage of $200 \mathrm{kV}$ with a current of $1 \mathrm{nA}$.

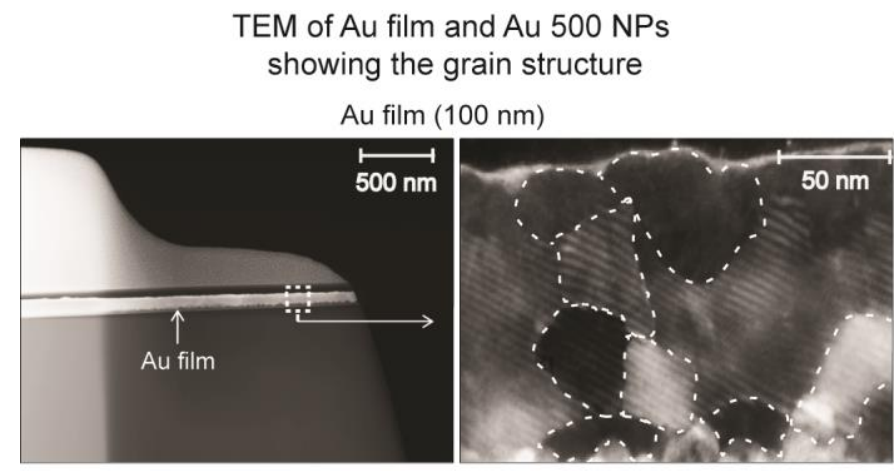

(a)

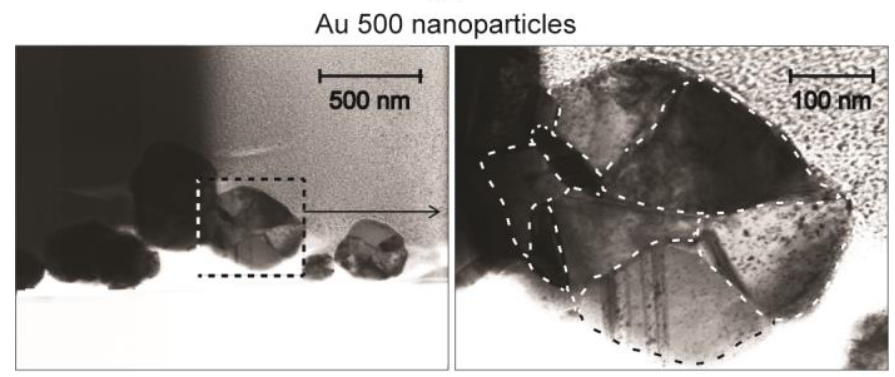

(b)

(Maharaj and Bhushan, 2014a)

Figure 9: (a) TEM images of (a) spherical Au nanoparticles approximately $50 \mathrm{~nm}$ in diameter (b) Au nanorods $50 \mathrm{~nm}$ in diameter and $200 \mathrm{~nm}$ in length (Nanopartz.com, 2015). 
Figure 10: TEM images of (a) $\mathrm{MoS}_{2}$ MWNT (Top), a magnified image showing separated layers between the arrows (Left), magnified image showing broken surface layers (Center) and a magnified image of a nanotube showing stacking faults highlighted by the dashed lines (Right) (b) agglomerated WS 2 MWNTS (Left), a magnified image with an arrow pointing to exfoliated layers (Center) and a magnified image of a nanotube showing ordered layers (Maharaj and Bhushan, 2015b). (c) Spherical CNHs (Left) with magnified image of CNHs (Center) and magnified image showing individual horns (Iijima et al., 1999). 
TEM of $\mathrm{MoS}_{2}, \mathrm{WS}_{2}$ and $\mathrm{CNH}$ nano-objects


$\mathrm{MoS}_{2}$ MWNTs showing
broken layers

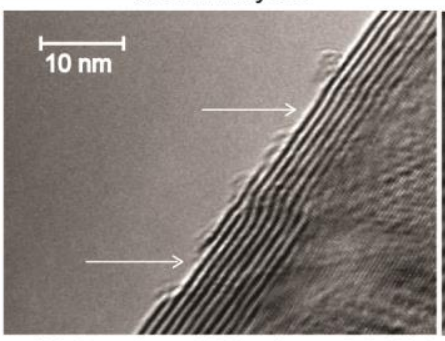

(Maharaj and Bhushan, 2015b)

(a)

$\mathrm{WS}_{2}$ MWNTs showing exfoliated layers

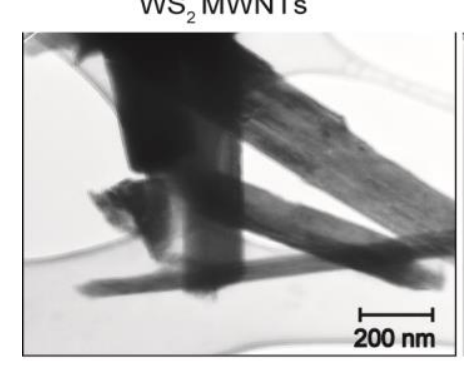

Spherical CNHs
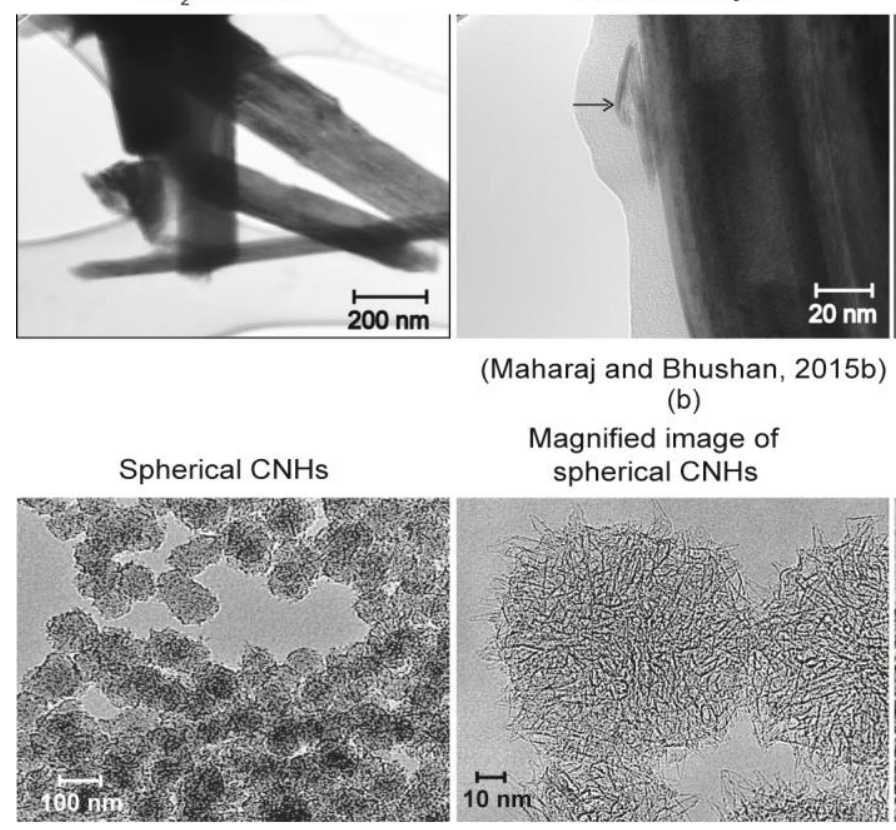

(Maharaj and Bhushan, 2015b)

(b)



(lijima et al., 1999)

(c)

Figure 10 
MWNTs from Weizmann Institute, Israel and were manufactured as discussed by Maharaj and Bhushan (2013). Diameters for $\mathrm{MoS}_{2}$ MWNTs can range from 40-500 nm and for WS 2 MWNTs, 40-300 nm with lengths from 0.5-20 $\mu \mathrm{m}$ for both. For CNHs horn shaped sheets of single-walled graphene $2-3 \mathrm{~nm}$ in diameter with a length of $30-50 \mathrm{~nm}$, agglomerate to form nanoparticles that are 80-100 nm in diameter. Figure 10a shows representative transmission electron microscopy (TEM) micrographs of a $\mathrm{MoS}_{2}$ nanotube with a broken edge (Top), a magnified image showing separated layers between the arrows (Left), magnified image showing broken surface layers (Center) and a magnified image of a nanotube showing stacking faults highlighted by the dashed lines (Right). Figure $10 \mathrm{~b}$ shows agglomerated $\mathrm{WS}_{2}$ nanotubes (Left), a magnified image with an arrow pointing to exfoliated layers (Center) and a magnified image of a nanotube showing ordered layers. For the micrographs in Figure 10a,b, the nano-objects are deposited on a lacey carbon support (Ted Pella, Redding, CA) and then placed in a holder and observed using a TEM system (Tecnai F20, FEI, Hillsboro, OR) operated at a voltage of $200 \mathrm{kV}$ with a current of $1 \mathrm{nA}$.

Single-walled graphitic CNHs (Meijo University, Japan) were created as described by Iijima et al. (1999) and Kasuya et al. (2002). ). CNHs are spherical nanoobjects made of single layers of graphene in the shape of horns. The horns agglomerate through van der Waals attraction to form the nano-object. Figure 10c shows TEM micrographs of spherical CNHs (Left) with a magnified image to the right (Iijima et al., 1999). 
For nano-object coated surfaces in dry environments, several droplets of $\mathrm{Au}$ nano-objects suspended in DI water, $\mathrm{MoS}_{2}$ and $\mathrm{WS}_{2} \mathrm{MWNTs}$ suspended in IPA and CNHs suspended in ethanol, were deposited onto clean Si (100) substrates using a syringe. The substrate was then placed on a hot plate and heated to a temperature of about $70-80{ }^{\circ} \mathrm{C}$ and left until the liquids evaporated.

The thin Au film was deposited onto the surface of the $\mathrm{Si}(100)$ substrate by thermal evaporation at an operating pressure of about $0.001 \mathrm{~Pa}$, substrate temperature of $100{ }^{\circ} \mathrm{C}$ at a rate of approximately $0.4 \mathrm{~nm} / \mathrm{s}$.

For submerged in liquid environments the MWNTs were sonicated for $3 \mathrm{~h}$ in water, dodecane and glycerol to aid dispersion whilst other nano-objects were sonicated for 15 mins. For Au nanoparticles which were already suspended in DI water, experiments were not carried out in dodecane and glycerol. When combined with glycerol agglomeration of the nano-objects occur and in the case of dodecane, its immiscibility with water prevented its use with the Au nanoparticles (Maharaj and Bhushan, 2012).

The dimensions of the various nano-objects used for each study are given along with descriptions of the approach for each study in the next section. 


\subsection{Friction and wear in dry and liquid environments: Au NPs and NRs, CNHs and $\mathrm{MoS}_{2}$ and $\mathrm{WS}_{2}$ MWNTs}

\subsubsection{Single nano-object contact}

\section{Nanoscale friction}

In single nano-object contact, as shown in Figure 3 as an example, a sharp tip with a nominal radius of $15 \mathrm{~nm}$ was used to manipulate the nano-object by lateral pushing. This allowed for determination of the friction force between the different nano-objects and substrate. A $0.005 \mathrm{mg} / \mathrm{ml}$ concentration was used to ensure that isolated unagglomerated nano-objects could be found and would not be hindered by other nano-objects during a manipulation attempt. The nominal diameters of Au nanoparticles used were $30 \mathrm{~nm}(\mathrm{Au}$ $30 \mathrm{NPs}$ ), $50 \mathrm{~nm}$ (Au $50 \mathrm{NPs}$ ), $90 \mathrm{~nm}$ (Au 90 NPs) (Nanopartz, Inc., Loveland, CO). Au nanorods $50 \mathrm{~nm}$ in diameter and $200 \mathrm{~nm}$ in length were also used (Au $50 \mathrm{NRs}$ ). The CNHs have nominal diameters of $80-100 \mathrm{~nm}$. For the $\mathrm{MoS}_{2}$ MWNTs nominal diameters were $45-75 \mathrm{~nm}$ with lengths of $0.7-1 \mu \mathrm{m}$ and for $\mathrm{WS}_{2}$ MWNTs the nominal diameters were 50$85 \mathrm{~nm}$ and also $0.7-1 \mu \mathrm{m}$ in length.

For friction forces, voltage data obtained were converted to forces by using an established method (Ruan and Bhushan, 1994a; Bhushan, 2011). The tip was first calibrated on a silicon surface by scanning along the cantilever axis to obtain the coefficient of friction and then orthogonal to the axis to obtain a conversion factor to change the friction signals to forces. Normal loads are determined by multiplying the cantilever vertical deflection by its stiffness (Bhushan, 2011). The vertical deflection in turn was obtained by operating the cantilever in force calibration mode, in which the 
deflection sensitivity obtained from the force curve was multiplied by the change in setpoint voltage. For manipulations where Au 90 and Au 30 NPs were compared, a sharp silicon tip (FORT series, Applied NanoStructures, Inc., Santa Clara, CA,) with a spring constant $(k)=3 \mathrm{~N} / \mathrm{m}$ and nominal radius of $15 \mathrm{~nm}$ was used for manipulation of a single Au nanoparticle under dry environments. For the other nano-object experiments, a sharp silicon nitride tip (Orc8 series, Bruker, Camarillo, CA) with $k=0.38 \mathrm{~N} / \mathrm{m}$ and a nominal radius of $15 \mathrm{~nm}$ was used for manipulation of single nano-objects in dry environments.

For submerged in liquid environments, a silicon nitride tip of lower force constant was used (Orc8 series, Bruker, Camarillo, CA) with $k=0.05 \mathrm{~N} / \mathrm{m}$ and a nominal radius of $15 \mathrm{~nm}$. A $0.01 \mathrm{mg} / \mathrm{ml}$ concentration of nano-objects was used. A droplet was placed in a fluid cell consisting of a standard multimode cantilever holder (Bruker, Santa Barbara, CA) with a glass plate glued just above the cantilever. The resulting meniscus bridge formed between the glass plate and substrate completely encloses the cantilever, which eliminates any viscous effects and adhesion due to meniscus forces. The average friction force value presented was the result of five manipulations.

\subsubsection{Multiple nano-object contact}

\section{Nanoscale friction and wear}

For multiple nano-object contact as shown in Figure 3 as an example, a glass sphere attached to an AFM tip was used to slide over several nano-objects. to determine the coefficient of friction, soda lime glass spheres (Duke Scientific Corporation, Palo Alto, CA) of nominal radii $15 \mu \mathrm{m}$ and $50 \mu \mathrm{m}$ attached to a silicon probe (FORT series, 
Applied NanoStructures, Inc., Santa Clara, CA) with a spring constant $(k)=3 \mathrm{~N} / \mathrm{m}$ was used. A $0.01 \mathrm{mg} / \mathrm{ml}$ concentration of nano-objects was used. For studies involving comparisons between Au 30 and Au 90 NPs a $15 \mu \mathrm{m}$ glass sphere was used and for all other studies a $50 \mu \mathrm{m}$ glass sphere was used. Friction and normal force data for nanoscale experiments were obtained by using the same calibration method as in single nano-object contact. Coefficient of friction data were obtained by plotting the friction force as a function of normal load from five random spots on the test samples.

For $\mathrm{Au} 50 \mathrm{NRs}$ contact pressure was determined to compare with that of macroscale in order to understand the scale effects in friction. A Hertzian contact analysis was performed. First, the number of NRs per unit area was found from analyzing topography images from wear tests, in sections outside of the wear area $\left(\sim 10 / \mu \mathrm{m}^{2}\right)$. This was done using SPIP 5.1.11 (Image Metrology A/S, Horshølm, Denmark). Second, the contact area on a flat gold surface is calculated for a given load $\left(0.0017 \mu \mathrm{m}^{2}\right.$ at $13 \mathrm{nN}$ and $0.0087 \mu \mathrm{m}^{2}$ at $150 \mathrm{nN}$ ) and knowing the particle density, the number of NRs for a given load under the glass sphere can be determined. Third, the contact pressure on a single NR is determined and this is divided by the number of NRs under the glass sphere to determine the overall contact pressure. Since number of nanorods under the tip is calculated to be as less than 1 , a single particle was taken for stress calculations. The contact pressure range was from $0.2 \mathrm{GPa}$ to $0.6 \mathrm{GPa}$ in the load range used. The relevant material parameters for the Hertzian analysis are given in Table A.2 (see Appendix A).

Wear experiments on the nanoscale were performed by using the glass sphere attached to a silicide coated cantilever (NANOSENSORS, Neuchatel, Switzerland) with $k$ 
$=40 \mathrm{~N} / \mathrm{m}$. In this case a cantilever of higher stiffness was used to obtain a normal load of $20 \mu \mathrm{N}$. Using a similar contact analysis as on the nanoscale, the contact pressure on the NRs was found to be $3.1 \mathrm{GPa}$. The normal load is obtained as described in the previous section. The wear test was carried out for 1,10 and 100 cycles at $10 \mathrm{~Hz}$, over a $10 \mu \mathrm{m}$ $\times 10 \mu \mathrm{m}$ scan size. A larger scan size of $20 \mu \mathrm{m} \times 20 \mu \mathrm{m}$ was then taken of the area enclosing the wear scar for comparison. SEM micrographs were also taken of the wear scars and areas within the wear scars to determine the condition of the nanoparticles and nanotubes. Tests were not performed in liquid environments, since, as the test is completed, nano-objects suspended in solution will continue to be deposited on the surface as the water evaporates and hence cover the wear area. Representative data for 5 -6 tests are summarized in the results section. All experiments were performed at room temperature $\left(23^{\circ} \mathrm{C}\right)$ and $50-55 \%$ relative humidity.

\section{Macroscale friction and wear}

For comparison to the nanoscale, macroscale friction tests were conducted using a ball-on-flat tribometer to determine if similar effects would be observed on both scales. A sapphire ball of $1.5 \mathrm{~mm}$ radius was fixed to a stationary holder attached to a cantilever beam. The substrate was loaded by use of a micrometer actuator which lowered the beam onto the substrate, after which tribometer was then operated in a reciprocating manner (Bhushan, 2002). The stroke length was $10 \mathrm{~mm}$ with an average speed of $3.5 \mathrm{~mm} / \mathrm{s}$. Normal and frictional forces were measured, with semiconductor strain gages mounted on the beam, for 500 cycles. In liquid environments, 2-3 droplets of liquid, with and 
without nano-objects were deposited onto the silicon substrate with a syringe. The nanoobject concentration was $0.01 \mathrm{mg} / \mathrm{ml}$. The sapphire ball was then slid over the substrate. The coefficient of friction was obtained as a function of number of cycles. Wear was characterized by taking optical and SEM micrographs of the wear scars created during the test.

Using a similar contact analysis as in section 3.2.2, the contact pressure on the Au 50 NRs was found to be $0.15 \mathrm{GPa}$

\subsection{Friction on single nano-objects: $\mathrm{MoS}_{2}$ and $\mathrm{WS}_{2}$ MWNTs}

For nanoscale friction studies (Figure 3) the diameters of the nanotubes used were at least $200 \mathrm{~nm}$ and lengths no less than $5 \mu \mathrm{m}$ for both $\mathrm{MoS}_{2}$ and $\mathrm{WS}_{2}$ MWNTs. Topography and friction maps were obtained with an AFM (Dimension 3100, Bruker, Santa Barbara, CA). A sharp silicon nitride tip (Orc8 series, Bruker, Camarillo, CA) with cantilever stiffness $k=0.1 \mathrm{~N} / \mathrm{m}$ and a nominal radius of $15 \mathrm{~nm}$ was used.

Friction maps were obtained at a constant normal load over scan sizes of $2 \mu \mathrm{m} \times 2 \mu \mathrm{m}$, $500 \mathrm{~nm} \times 500 \mathrm{~nm}$ and $5 \mathrm{~nm} \times 5 \mathrm{~nm}$ (Maharaj and Bhushan, 2015a). For coefficient of friction, voltage data obtained were converted to forces by using an established calibration method (Ruan and Bhushan, 1994a; Bhushan, 2011) as discussed in the section on single nano-object contact The coefficients of friction for the nanotubes were then obtained by plotting the friction force obtained during orthogonal scanning as a function of normal load from five random spots over a scan length of $5 \mathrm{~nm}$ and velocity of $9 \mathrm{~nm} / \mathrm{s}$. The scan length is very small compared to the minimum diameters of the 
nanotubes $(200 \mathrm{~nm})$ and removes any curvature effects on friction. Normal loads were obtained as discussed for single nano-object contact.

\subsection{Mechanical behavior: Au thin film, NPs and NRs, CNHs and $\mathrm{MoS}_{2}$ and $\mathrm{WS}_{2}$ MWNTs}

\subsubsection{Nanoindentation with a Berkovich tip}

All experiments were carried out using a probe based scanning nanoindenter head TS 75 Trboscope, (Hysitron, Inc., Minneapolis, MN) attached to an AFM (Dimension 3100, Bruker, Santa Barbara, CA) with a diamond tip. For nanoindentation experiments a three-sided diamond pyramidal Berkovich tip of approximately $100 \mathrm{~nm}$ in radius was used as shown in Figure 3. The Oliver and Pharr (1992) method was used to obtain the hardness and elastic modulus. A reduced modulus is obtained from the slope of the unloading part of the load-displacement curve. This is a function of the Young's moduli and Poisson's ratios of diamond and the nanomaterial of interest. The Young's modulus of elasticity and Poisson's ratio for diamond were taken as $1141 \mathrm{GPa}$ and 0.07 respectively (Harper, 2001). With the known Poisson's ratio for the nanomaterial the unknown Young's modulus can now be determined. Hardness is found from the ratio of the maximum load to the contact area. For Au and $\mathrm{MoS}_{2}$, Poisson's ratio was taken as 0.42 (Samsonov, 1968) and 0.27 (Feldman, 1976) respectively. For all indentation experiments contact depth does not exceed 30\% of the nano-object height or film thickness, to prevent substrate hardening effects (Anonymous, 1979). All experiments were performed at room temperature $\left(23{ }^{\circ} \mathrm{C}\right)$ and $50-55 \%$ relative humidity. 


\section{Au thin film and NPs}

Hardness and elastic modulus were obtained as a function of contact depth for the $100 \mathrm{~nm}$ thin film by indenting at maximum loads of 20, 40, 60 and $80 \mu \mathrm{N}$. The $20 \mu \mathrm{N}$ load was the lowest load that was possible to produce well defined load displacement curves for analysis. The $80 \mu \mathrm{N}$ load was the maximum load possible without the substrate influencing

the values of hardness. The data from these experiments is the average of five measurements for each load.

Effects of creep were studied at 40 and $80 \mu \mathrm{N}$ using a hold period of 200s at the maximum loads. Strain rate effects were investigated at a load of $80 \mu \mathrm{N}$ for loading and unloading times of 20 and $200 \mathrm{~s}$. The creep and strain rate data gives an indication of whether the experiments are sensitive to hold times and loading-unloading rates and helps to further characterize mechanical behavior which is important for determining suitability for various applications.

For nanoparticle experiments, Au 500 NPs were indented at maximum loads of 20, 40, 60 and $80 \mu \mathrm{N}$ similar to the thin film. The large diameter of the nanoparticle compared to the $100 \mathrm{~nm}$ radius of the sharp tip provides as flat a surface for nanoindentation of a nano-object. This allows for reduction of curvature effects. As nanoparticle size decreases there is less contact with the tip due to the curvature of both the tip and nanoparticle and this can lead to inaccurate determination of contact area. This results in errors in obtaining mechanical properties accurately. The data from these experiments is the average of five measurements on five different nanoparticles for each 
load. The duration for loading and unloading was $20 \mathrm{~s}$ for all experiments (unless otherwise stated) to safeguard against the nanoparticle slipping under the indenter during more rapid and unstable loading. Topography images were also taken before and after indentation with the same tip used for indentation.

To measure the grain sizes of the film and Au 500 shown in Figure 9, the average grain diameters were found by first importing the TEM images into an image processing and analysis software (Image J, National Institute of Health, Bethesda, MD). Second, the outlines of the grains were traced and the enclosed area was determined. The outlines were visible due to the difference in color from one grain to the next as a result of the misalignment of atoms at the grain boundary. Using the area, the diameter of a circle of equivalent area is found and this is taken as the grain diameter. The average grain diameters from the representative images were found to be $40 \pm 9 \mathrm{~nm}$ for the film and 96 $\pm 30 \mathrm{~nm}$ for the nanoparticle.

For comparison to the macroscale, data from polycrystalline bulk Au was used from experiments presented by Lozinskii (1961). Vickers hardness was obtained using a four sided diamond tip under a load of $1 \mathrm{~kg}$. Typical samples were disc shaped with a diameter of $14.8 \mathrm{~mm}$ and thickness of $5 \mathrm{~mm}$. The Young's modulus for bulk was obtained through resonance of transverse vibrations of a cylindrical specimen which was typically $100 \mathrm{~mm}$ in length and 6-8 $\mathrm{mm}$ in diameter (Lozinskii, 1961). 


\section{$\mathrm{MoS}_{\mathbf{2}}$ MWNTs}

In these experiments $\mathrm{MoS}_{2} \mathrm{MWNTs} \sim 500 \mathrm{~nm}$ in diameter with lengths of $15-20 \mu \mathrm{m}$ were selected. Similar to Au 500 NPs, the large diameter of the nanotubes compared to the $100 \mathrm{~nm}$ radius of the sharp tip provides a flatter surface for nanoindentation compared to $\mathrm{WS}_{2}$ MWNTs and CNHs. This reduces curvature effects and errors in obtaining mechanical properties accurately. This was not possible with the other nano-objects due to their smaller dimensions. In determination of mechanical properties a displacement of $50 \mathrm{~nm}$ was selected. Hardness and elastic modulus were obtained by indenting at maximum displacement of $50 \mathrm{~nm}$ perpendicular to the basal plane to avoid substrate hardening effects. Displacements of $100 \mathrm{~nm}$ were also explored and were the maximum displacements possible before the Berkovich tip penetrated the walls of the nanotube into the hollow interior. This was done to explore deformation under increased depths of penetration. The data from these experiments is the average of five measurements on five different nanotubes for each load. The duration for loading and unloading was $20 \mathrm{~s}$ for all experiments to safeguard against the nanotube slipping under the indenter during more rapid and unstable loading. Topography images were also taken before and after indentation with the same tip used for indentation.

\subsubsection{Compression with a flat punch}

For compression experiments a spherical diamond tip of approximately $3.5 \mu \mathrm{m}$ in radius was used as shown in Figure 3. This can be considered to be a flat punch due to the large radius of the diamond tip compared to the nanoparticles. Figure 11 shows the 
topography map (top left) and corresponding 2-D profile (bottom left) of the flat punch. The pairs of arrows indicate the section on which the profile is taken. The dashed lines represent the sides of the holder on which the tip is glued. The single arrow points to a representative $200 \mathrm{~nm} \times 200 \mathrm{~nm}$ section on the punch which is illustrated by the 3-D map (right). The root mean squared roughness $(\mathrm{RMS})=0.5 \mathrm{~nm}$ and the peak to valley $(\mathrm{P}-\mathrm{V})$ roughness $=5.9 \mathrm{~nm}$. The low roughness allows for overall compression of the nanoparticles without indentation due to any large asperities which may be present on the surface.

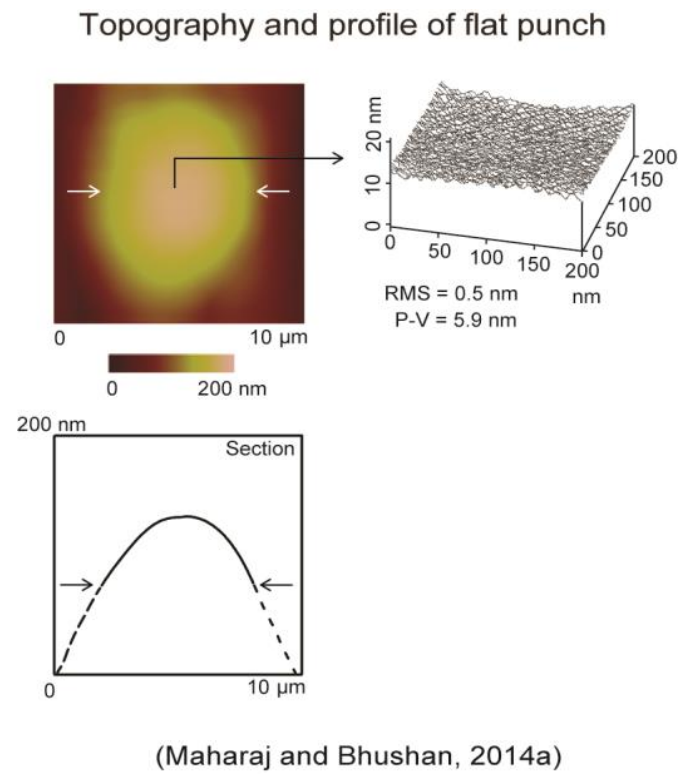

Figure 11: Topography map (top) and 2-D profile (bottom) of flat punch at the section shown by the pairs of horizontal arrows along with a typical line plot (right) of a $200 \times 200 \mathrm{~nm}$ section indicated by the single horizontal arrow (Maharaj and Bhushan, 2014a). 


\section{Au NPs and NRs}

For Au 50 NPs and Au 50 NRs maximum loads of 50 and $200 \mu \mathrm{N}$ known as low and high were applied to the nano-objects. Repeat compression loading experiments, where several loads are applied to a single nanoparticle, were also performed. Experiments were carried out to explore strain hardening effects on the nanoscale as well as pop-in behavior. The range was $80-110 \mu \mathrm{N}$ and loads were applied in increasing increments of $20 \mu \mathrm{N}$ to obtain enough load-displacement curves to clearly observe strain hardening. The range was limited by the nano-objects either being pushed during imaging or stuck to the diamond tip during compression. This makes imaging and location of the nanoparticle impossible for further compression. The duration for loading and unloading was $20 \mathrm{~s}$ for all experiments.

For the Au $500 \mathrm{~nm}$ nanoparticle compression was performed at a maximum load of $80 \mu \mathrm{N}$ similar to indentation. Repeat compression loading experiments were also performed. The range was $50-250 \mu \mathrm{N}$ and loads were applied in increasing increments of $50 \mu \mathrm{N}$ to obtain enough load-displacement curves to clearly observe strain hardening.

To ensure repeatability, each experiment was performed five times and representative data are shown in the results section. Experiments were performed at room temperature $\left(23{ }^{\circ} \mathrm{C}\right)$ and $50-55 \%$ relative humidity. 


\section{$\mathrm{MoS}_{2}$ and $\mathrm{WS}_{2}$ MWNTs and $\mathrm{CNHs}$}

Compression was performed under displacement control for displacements of 50, 100 and $300 \mathrm{~nm}$ for the $\mathrm{MoS}_{2}$ and $\mathrm{WS}_{2}$ MWNTs. These displacements were selected to understand how the nano-objects deform under various levels of compression. The lowest displacement was $50 \mathrm{~nm}$ similar to indentation. This was done to compare the deformation between indentation and compression and to investigate global deformation for $\mathrm{MoS}_{2}, \mathrm{WS}_{2} \mathrm{MWNTs}$ and CNHs. For the CNHs displacements were 25, 50 and $75 \mathrm{~nm}$ due to smaller diameters of the nanoparticles which ranged from 80-100 nm. Repeat compression loading experiments, where the nano-objects are loaded several times for a fixed displacement, were performed. Experiments were carried out to explore hardening effects on the nanoscale as well as pop-in behavior. For the nanotubes the displacement was held constant at $50 \mathrm{~nm}$. The number of compressions was limited by the nanotubes either being pushed during imaging or stuck to the diamond tip during compression. This makes imaging and location of the nanotube impossible for further compression. For the $\mathrm{CNHs}$ the displacement was held constant at $25 \mathrm{~nm}$. The duration for loading and unloading was $20 \mathrm{~s}$ for all experiments similar to nanoindentation. Topography images were also taken before and after indentation with the same tip used for compression.

To ensure repeatability, each experiment was performed five times and representative data are shown in the results section. Experiments were performed at room temperature $\left(23{ }^{\circ} \mathrm{C}\right)$ and $50-55 \%$ relative humidity for the nanotubes and for the $\mathrm{CNHs}$ the relative humidity was $70-75 \%$ to ensure that the nanoparticles were securely held in place by meniscus forces due to its lubricious nature. 


\title{
Chapter 4: Friction and wear in dry and liquid environments: Au NPs and NRs
}

\begin{abstract}
4.1 Introduction
In this section, results from experiments in single and multiple nano-object contact are presented for dry environments and submerged in liquid environments for water, dodecane and glycerol by Maharaj and Bhushan $(2012,2014 b)$ for the first time. The friction forces are presented for $\mathrm{Au}$ 30, Au 50 and Au 90 NPs and Au 50 NRs. In addition, wear data on the nanoscale and macroscale with and without addition of nano-objects are also presented. Morphological characterization of the nano-objects and wear scars are also shown.
\end{abstract}

\subsection{Single nano-object contact}

\subsubsection{Manipulation technique}

For single nano-object contact in under dry environments, a sharp tip is used to manipulate Au nanoparticles by pushing in the lateral direction. Figure 12 shows an example of topography images of nanoparticles highlighted by the squares, before and after manipulation within the same scan area for a Au 90 NP. A normal load of $10 \mathrm{nN}$ was used during imaging. This takes place on the scan line illustrated by the black arrows. A $2 \mu \mathrm{m} \times 2 \mu \mathrm{m}$ scan area is used to ensure that the nanoparticle can still be seen 
in the same image after manipulation. This clearly shows that the nanoparticles are being moved by the AFM tip.

Figure 13 shows examples of (a) topography maps and height profiles of the scan region of interest before manipulation and after manipulation and (b) 2-D friction force

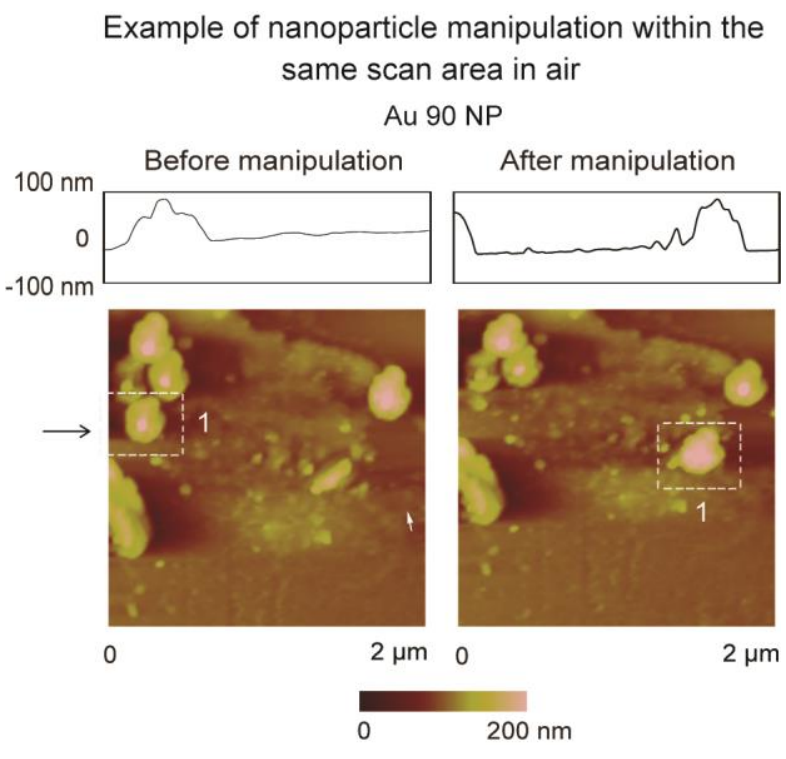

(Adapted from Maharaj and Bhushan, 2012)

Figure 12: Example of topography maps and height profiles, at sections shown by the arrows, of a Au nanoparticle $90 \mathrm{~nm}$ in diameter, highlighted by the squares, manipulated within the same scan area before and after manipulation (Maharaj and Bhushan, 2012).

profiles for a Au $90 \mathrm{NP}$. A $1 \mu \mathrm{m}$ x $1 \mu \mathrm{m}$ area is imaged before manipulation to identify nanoparticles of interest as seen in Figure 13a (Left). During manipulation in the same scan area, as shown in Figure 13b, scanning proceeds in the slow scan direction (bottom 
Figure 13: (a) An example of a topography image and height profile showing the same scan area before and after manipulation (b) Topography maps and 2-D friction force profiles, of a Au 90 NP during manipulation of single nanoparticles in air (dry environment). During manipulation, the normal load is increased (AB) halfway through the scan of the nanoparticle (indicated by the black arrows) from $10 \mathrm{nN}$ to $300 \mathrm{nN}$ on a single scan line. On this scan line, the sharp tip slides laterally (B-C) until contact is made with the particle. The lateral friction force (C-D) increases, as the sharp tip pushes against the particle, until it begins to move. E shows the end of the scan line (Maharaj and Bhushan, 2012). 


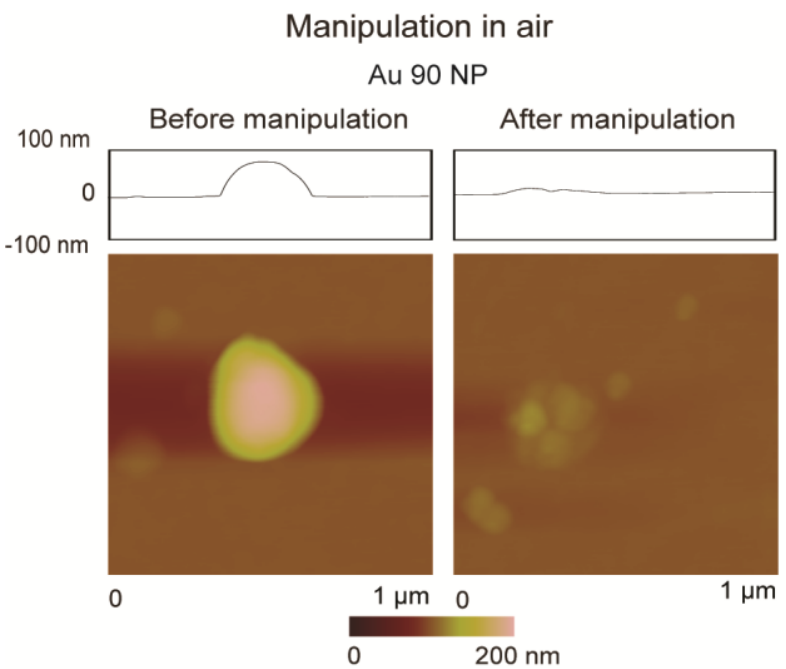

(a)


(b)

(Adapted from Maharaj and Bhushan, 2012)

Figure 13 
to top), and the normal load is increased from $10 \mathrm{nN}$ to $300 \mathrm{nN}$ at the approximate center of the nanoparticle. This corresponds to an increase in the friction signal (A-B), on a single scan line as illustrated by the black horizontal arrows. Increasing the normal load prevents the nanoparticle from being imaged as the cantilever tip remains in contact with the substrate and does not slide over the nanoparticle to track its height. As the sharp tip continues to slide along the scan line from left to right (fast scan direction), there is a further increase in the friction signal (C-D) which directly correlates to the twisting of the cantilever as it pushes against the nanoparticle, until friction is overcome and sliding begins. Point E represents the end of the scan line on which the manipulation takes place. A profile and topography map after nanoparticle manipulation is shown in Figure. 13a (Right), which provides proof that the nanoparticle is pushed out of the scan area. Imaging of the nanoparticle was done in contact mode for both pre- and postmanipulation. The change in lateral force (C-D) is used to quantify the friction between the nanoparticle and silicon surface as sliding is initiated.

For submerged in water environments as shown in Figure 14, a $10 \mu \mathrm{m}$ x $10 \mu \mathrm{m}$ area is imaged at a normal load of $1 \mathrm{nN}$. This allows for multiple manipulations within a single topography scan. As an example, a single partial image of a nanoparticle, highlighted by the squares is used to demonstrate the manipulation technique. The associated topography and friction force scan lines, before manipulation (top), during manipulation (middle) and after manipulation (bottom) for the above mentioned nanoparticle are also shown. In this case the normal load does not have to be increased since the nanoparticles can be pushed due to the low adhesion between them and the 
Figure 14: Topography map of a Au 90 NP submerged in water. Imaging is performed using a cantilever with a low normal load of $1 \mathrm{nN}$. The highlighted particle is examined and the associated topography and friction force scan lines before manipulation, during manipulation and after manipulation are shown. Before manipulation, there is a rise in friction force corresponding to the nanoparticle profile as the cantilever deflects during imaging. During manipulation the topography profile is flat as the particle is being pushed which corresponds to a rise in friction force (A-B). After manipulation, the topography and friction force profiles remain flat as the particle is no longer there (Maharaj and Bhushan, 2012). 


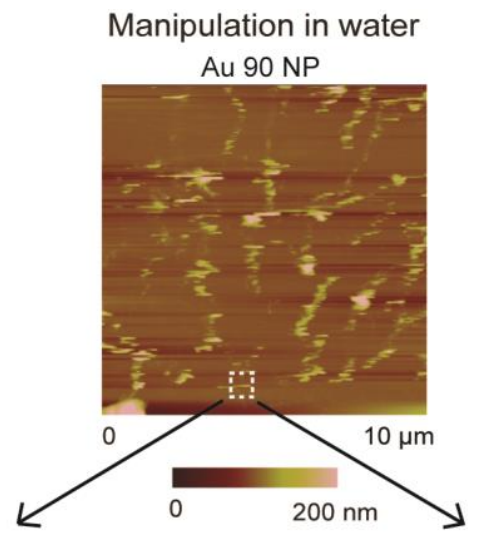

Before manipulation ( $1{ }^{\text {st }}$ scan line) (Normal load, $1 \mathrm{nN}$ )


During manipulation ( ${ }^{\text {nd }}$ scan line)



After manipulation ( $3^{\text {rd }}$ scan line)



(Adapted from Maharaj and Bhushan, 2012)

Figure 14 
substrate during scanning. As the nanoparticle is imaged, there is a rise in friction force associated with twisting of the cantilever (top) corresponding to the nanoparticle profile. In the middle set of scan lines, the topography is flat since the tip no longer follows the nanoparticle profile as it is being pushed, which corresponds to an increase in friction force. This increase (A-B) corresponds to the friction force between the Au nanoparticle and the silicon substrate at the initiation of sliding. In the bottom set of scan lines, both the topography and friction force scan lines remain flat which proves that the nanoparticle has been pushed out of the area. The friction force results for nanoparticle manipulation in dry and submerged in liquid environments is presented and discussed in the following section.

\subsubsection{Nanoscale friction}

Friction forces during nano-object manipulation are shown in Figure 15a for $\mathrm{Au}$ 30 and Au 90 NPs along with Au 50 NPs and Au 50 NRs in dry environments (Maharaj and Bhushan, 2012, 2014b). Figure 15a also compares the friction force when the normal load is $150 \mathrm{nN}$ and $300 \mathrm{nN}$. The friction forces are comparable as is expected, since the normal load should only influence the interaction between the tip and the substrate. The friction force between the Au nanoparticle and the substrate provides the additional offset C-D in the friction signal shown in Figure 13b during manipulation in air, independent of the tip-substrate friction force (Dietzel et al., 2007). If the tip-substrate friction force approaches that of the nano-object-substrate force, it is expected that the friction force 
signal during manipulation would be masked. This would necessitate lowering of the normal load as in the case of nano-objects submerged in the water.

In Figure 15a higher friction forces as observed with Au 90 NPs compared to Au 30 NPs and Au 50 NRs compared to Au 50 NPs. As discussed by Maharaj and Bhushan (2012), the normal load acting on the NPs and NRs is due only to the mass of the nanoobject since it is pushed from the side and the friction force is the result of adhesion between the nano-object and the silicon substrate. The adhesive force can include van der Waals forces in both dry and submerged in water environments and meniscus forces in dry environments. In this regime the friction force is not proportional to the normal load since it is dependent on the contact area. Since the adhesive force is dependent on surface area, the larger nano-objects will display higher friction forces.

Figure $15 \mathrm{~b}$ presents the result of measurements of the average friction force for Au 30 and Au 90 NPs along with Au 50 NPs and Au 50 NRs submerged in water environments. The vertical scale is magnified for data in water in order to discern the difference in friction force. Since the high normal loads, used during manipulation, in 
Figure 15: Friction force in (a) air during manipulation of Au 90 NPs, at normal loads of $150 \mathrm{nN}$ and $300 \mathrm{nN}$ (Left) (Maharaj and Bhushan, 2012) and Au 50 NPs and NRs at normal loads of $300 \mathrm{nN}$ on the silicon substrate (Right) (Adapted from Maharaj and Bhushan, 2014b. (b) In water at a normal load of $1 \mathrm{nN}$ for Au 90 NPs, (Left) (Maharaj and Bhushan, 2012) and Au 50 NPs and NRs on the silicon substrate (Right) (Maharaj and Bhushan, 2014b). The vertical scale is magnified for the data in water. (c) Coefficients of friction on the nanoscale for both dry and submerged in water environments, with and without nanoobjects for (a) Au 30 and Au 90 (Left) (Maharaj and Bhushan, 2012) and Au 50 NPs and NRs (Right) (Maharaj and Bhushan, 2014b). Friction forces and coefficient of friction are lower in submerged in water environments (Maharaj et al., 2013). 
Friction force (During manipulation in air) Sharp tip $\sim 15 \mathrm{~nm}$

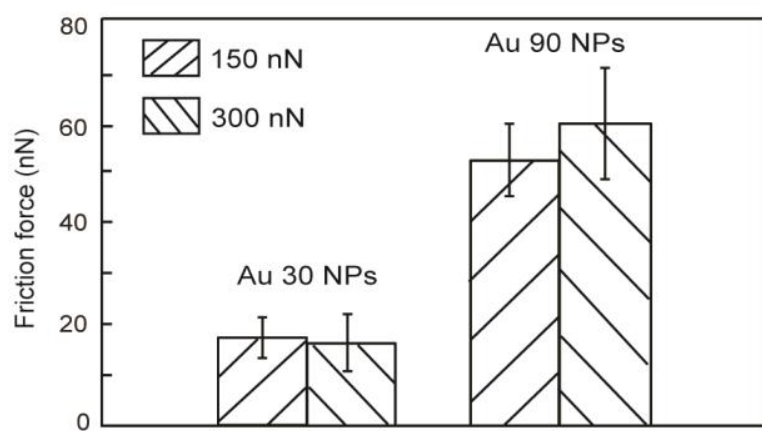

(Maharaj and Bhushan, 2012)

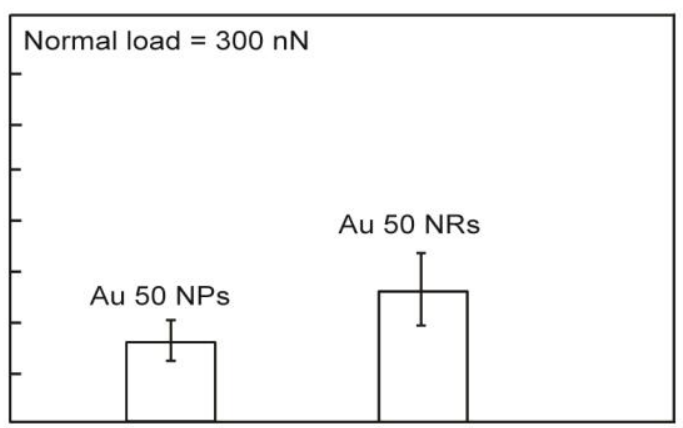

(Adapted from Maharaj and Bhushan, 2014b)

(a)

Friction force (During manipulation in water) Sharp tip $\sim 15 \mathrm{~nm}$

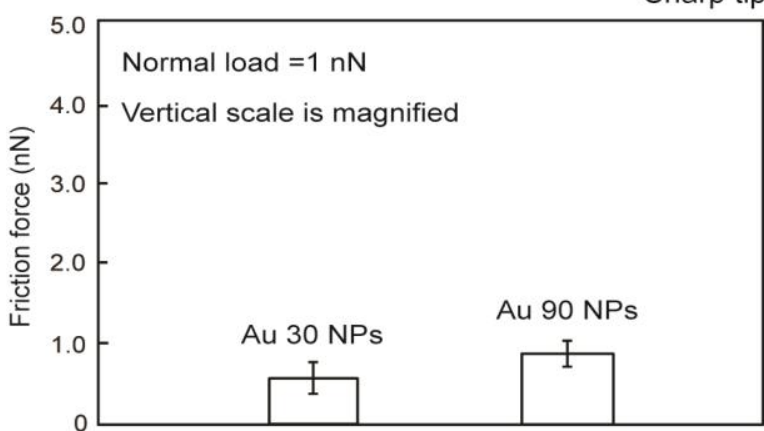

(Maharaj and Bhushan, 2012)
Normal load $=1 \mathrm{nN}$

Vertical scale is magnified

(b)

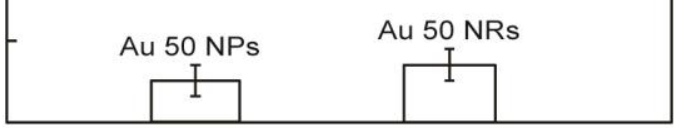

(Maharaj and Bhushan, 2014b)

Coefficient of friction (During sliding on nano-objects)


(c)

Figure 15 
dry environments would mask the friction force signal, a lower normal load of $1 \mathrm{nN}$ is used for nanoparticle manipulation. This is sufficient since the nanoparticles are weakly adhered to the substrate and can be easily moved during the manipulation process shown in Figure 14. The adhesive forces are due to van der Waals interactions since there are no meniscus bridges formed in submerged in water environments. The lower friction forces in the submerged in water environments compared to the dry environments can thus be attributed to the elimination of meniscus forces and sliding on a surface of low shear strength. The lower friction forces occur with Au 30 and Au 50 NPs due to the lower contact area, independent of an applied normal load.

\subsection{Multiple nano-object contact}

\subsubsection{Nanoscale friction and wear}

In multiple nano-object contact, the effect of $\mathrm{Au} 30$ and $\mathrm{Au} 90$ NPs along with $\mathrm{Au}$ 50 NPs and Au 50 NRs on friction and wear reduction is studied.

Figure 15 summarizes the coefficient of friction for dry and submerged in water environments. Sliding in multiple nano-object contact results in lower coefficients of friction for all cases compared to sliding on the bare silicon substrate. The coefficient of friction reduction can be attributed to the mobility of the nano-objects in addition to the reduced contact area. As discussed by Maharaj and Bhushan (2012, 2013, 2014b), it is expected that as the glass sphere comes into contact with the nano-objects, some of them

are deformed, since the larger nano-objects would be encountered first and experience the highest contact pressures, due to fewer nano-objects to support the normal load. The 
resulting friction reduction mechanism can thus be attributed to the reduced contact area, the sliding over deformed nano-objects as well as the individual nano-objects dragged with the glass sphere as shown in Figure 16. As the glass sphere encounters a greater number of nano-objects, the contact pressure is reduced allowing undeformed nanoobjects to roll between the surfaces as shown in Figure 16a. In this case, for Au 30, Au 90 and $\mathrm{Au} 50 \mathrm{NPs}$ there is a greater probability of rolling compared to Au 50 NRs. This is due to the geometry of the Au 50 NRs which allows it to roll if the NR is oriented approximately perpendicular to the direction of sliding of the glass sphere. In dry environments, as in single nano-object contact for the Au 30 NPs, the reduced contact area is responsible for the lower coefficient of friction compared to Au 90 NPs. The same argument is made for Au 50 NPs compared to Au 50 NRs along with having a higher probability of rolling. The reduced contact area also reduces load independent adhesion arising from the effects of van der Waals and meniscus forces as there is less asperity contact between the glass sphere and the silicon substrate.

In submerged in water environments, the coefficients of friction were lower compared to dry environments. Due to the low viscosity of water and the low speed of 20 $\mu \mathrm{m} / \mathrm{s}$ of the glass sphere, it is expected that the system operates in the boundary lubrication regime. The elimination of the meniscus forces together with sliding on a surface of low shear strength (Bhushan and Sundararajan, 1998) due to a thin water film results in overall lower coefficients of friction compared to dry environments for all nano-objects. Despite the higher contact area for the Au 90 NPs compared to Au 30 NPs and $\mathrm{Au} 50$ NRs compared to Au 50 NPs the coefficient of friction for both cases are 
Friction and wear reducing mechanisms during

sliding on a nano-object coated surface

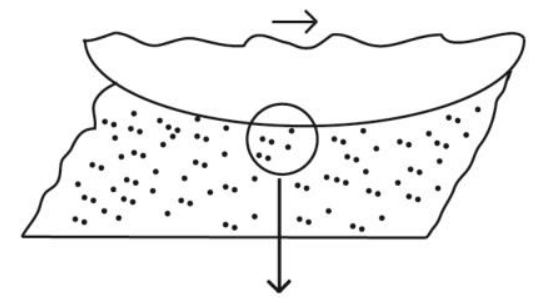

Mechanisms for spherical and tubular nano-objects with

$\mathrm{CNH}$ s nanoparticles used as an example

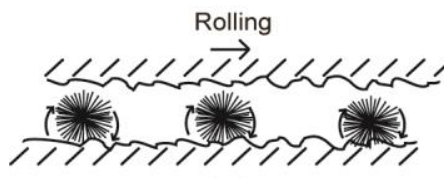

(a)



(b)

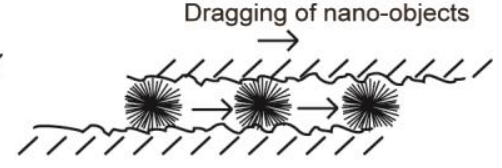

(c)

Mechanisms for nano-objects made of lamellar materials with nanotubes used as an example



(d)

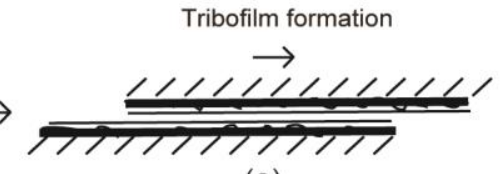

(e)

(Maharaj et al., 2013)

Figure 16: Schematic of friction and wear reducing mechanism of nano-object coated surface by (a) rolling, (b) sliding over nano-objects, (c) dragging of nanoobjects, (d) exfoliation, (e) and tribofilm formation. CNHs are used as an example for mechanisms (a) through (c) and $\mathrm{WS}_{2}$ and $\mathrm{MoS}_{2} \mathrm{MWNT}$ are used as an example for mechanisms (d) through (e) (Maharaj et al., 2013).

similar. This is due to the fact that the water provides a surface that is easily sheared and sliding is more likely to be initiated before rolling. This, together with the elimination of contact area dependent meniscus forces allows the for similar friction reduction. In addition, compared to single nano-object contact, sliding on several nano-objects can 
result in some agglomeration which contributes to a reduced ability to roll for all nanoobjects.

The ability to reduce wear of the underlying surface, in addition to reducing coefficient of friction, must also be considered for a prospective lubricant. Figure 17 summarizes the wear data for sliding on Si and Si coated with Au 50 NPs and Au 50 NRs used as examples. This was done for 1,10 , and 100 cycles under a normal load of $20 \mu \mathrm{N}$ in dry environments. Tests were not performed in liquid environments, since, as the test is completed, nano-objects suspended in solution will continue to be deposited on the surface as the water evaporates and cover the wear area. A $20 \mu \mathrm{m} \times 20 \mu \mathrm{m}$ area is imaged to show wear scars which are created over a $10 \mu \mathrm{m} \times 10 \mu \mathrm{m}$ area. Topography maps along with corresponding height profiles are also shown.

After 1 cycle the surface of the uncoated silicon appears rough during the early stages of wear as it becomes damaged (Maharaj and Bhushan, 2014b). For the NPs and NRs coated surface, after 1 cycle the nano-objects are just beginning to be pushed out of the wear area. After 10 cycles the topography profile of the uncoated silicon surface appears smoother than after 1 cycle. This is likely due to breaking and removal of sharp asperities and eventual polishing of the surface (Maharaj and Bhushan, 2014b). For the coated surfaces, the NPs and NRs agglomerate around the edges of the wear scar. For the uncoated silicon substrate after 100 cycles a small amount of material is removed with a wear depth of approximately $0.3 \mathrm{~nm}$ as seen in the height profile. For the coated surfaces the nano-objects are completely removed. 

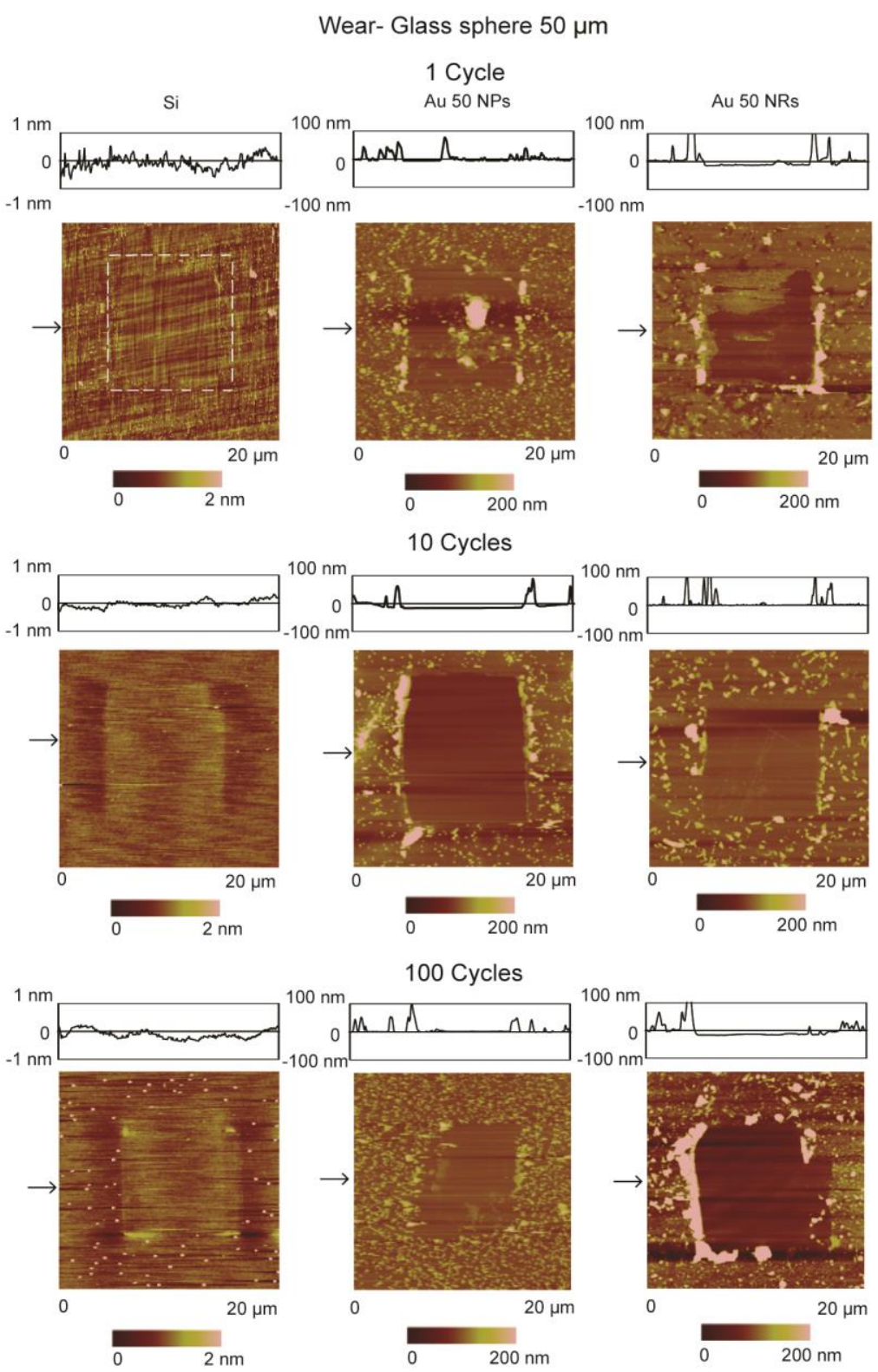

(Maharaj and Bhushan, 2014b)

Figure 17: Topography maps and 2-D profiles for nanoscale wear experiments, at sections shown by the arrows, after sliding at 1,10 and 100 cycles with a normal load of $20 \mu \mathrm{N}$ at $10 \mathrm{~Hz}$ on Si and Si coated with Au 50 NPs and NRs (Maharaj and Bhushan, 2014b). 
Figure 18 shows SEM micrographs of the wear scars in dry environments (First row) after 100 cycles.

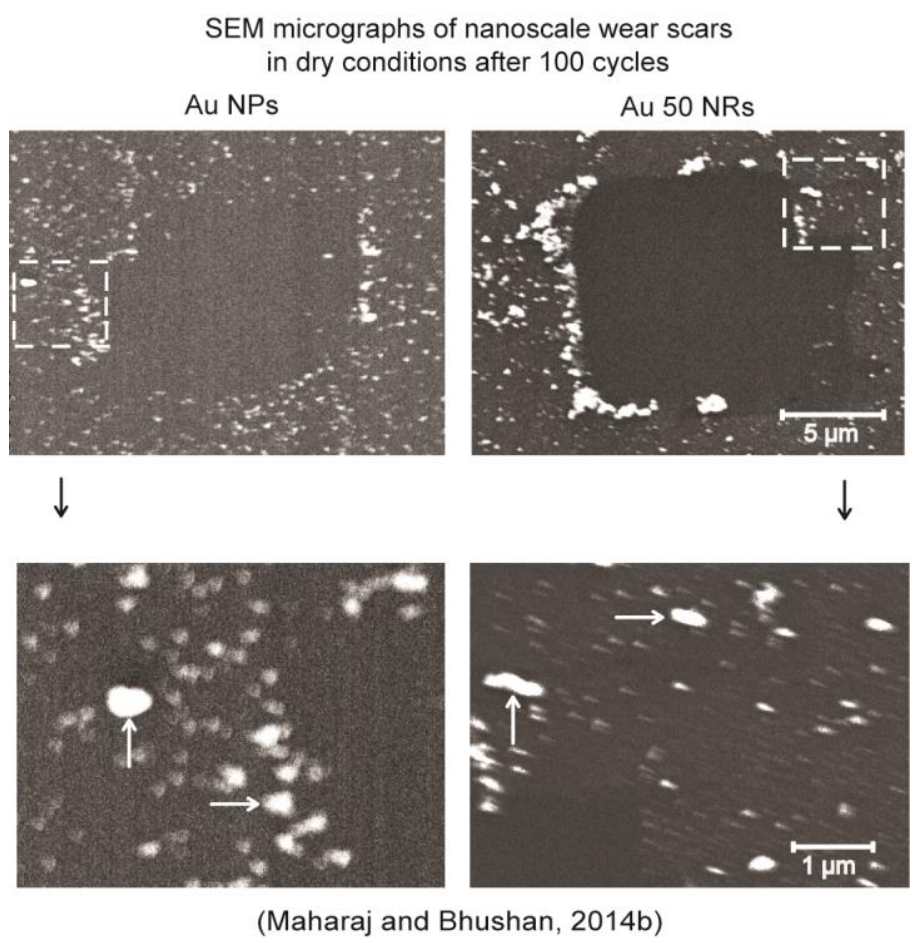

Figure 18: SEM micrographs of nanoscale wear scars for Au 50 NPs and NRs in dry conditions after 100 cycles at a normal load of $20 \mu \mathrm{N}$ (First row). The nanoobjects within the squares are magnified and the vertical arrows point to those micrographs in the second row. The magnified micrographs show agglomerated $\mathrm{Au} 50$ NPs and NRs as indicated by arrows within the micrographs (Maharaj and Bhushan, 2014b).

Magnified micrographs of the areas within the squares are shown in the second row as indicated by the vertical arrows. In the magnified micrographs wear debris shows 
agglomerated NPs and NRs are pointed out by arrows within each micrograph. This

debris consists of mostly deformed Au nano-objects with very little silicon. As mentioned in the previous paragraph and shown in Figure 18 only a small amount of silicon is removed from the bare substrate.

It is believed, since the nano-objects remain in the wear area after 1 cycle, that the damage of the silicon surface should be less than that of an initially uncoated substrate. This occurs since the nano-objects are believed to roll and slide between the glass sphere and the substrate which promotes facile shearing of the two surfaces as shown in Figure 16a-c, in addition to the reduced contact area provided by the nano-objects. The NRs, due to their larger surface area compared to the NPs will have a lower contact pressure acting on them and would be better able to support the applied load and provide more resistance to deformation over a longer period of time. After100 cycles, it is therefore expected that the greatest wear occurs on the bare silicon substrate and the least wear occurs with the NRs.

\subsubsection{Macroscale friction and wear}

In macroscale friction and wear tests a sapphire ball with a radius of $1.5 \mathrm{~mm}$ was slid over Au 50 NPs and Au 50 NRs coated and uncoated silicon substrates under a normal load of $200 \mathrm{mN}$. This was carried out for 500 cycles over a stroke length of 10 $\mathrm{mm}$ and velocity of $3.5 \mathrm{~mm} / \mathrm{s}$.

Optical micrographs of wear scars under dry and water environments with and without the nano-objects are shown in Figure 19a. In general, the widths of the wear 
Figure 19: (a) Optical micrographs of macroscale wear scars taken after 500 cycles at a normal load of $200 \mathrm{mN}$, for friction tests, in dry and water environments with and without Au 50 NPs and NRs. The sliding speed was $3.5 \mathrm{~mm} / \mathrm{s}$. (b) SEM micrographs of the wear scar for agglomerated Au 50 NPs and NRs in dry conditions shown within the squares (Maharaj and Bhushan, 2014b). The least wear occurs with nano-objects in water. 
Optical micrographs of macroscale wear scars

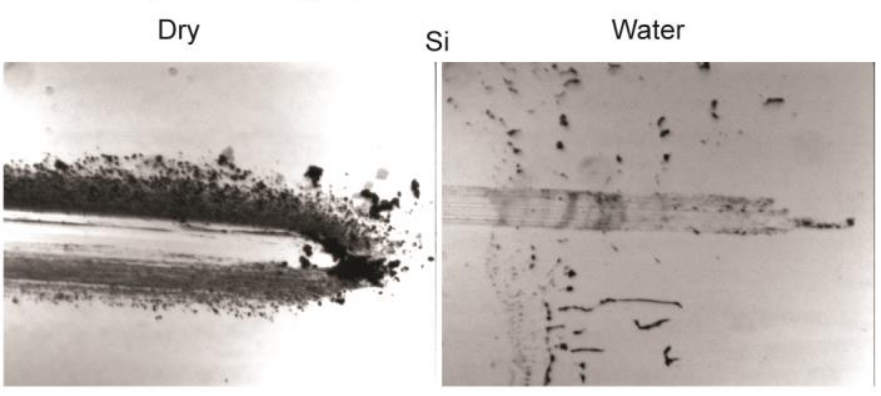

Au 50 NPs
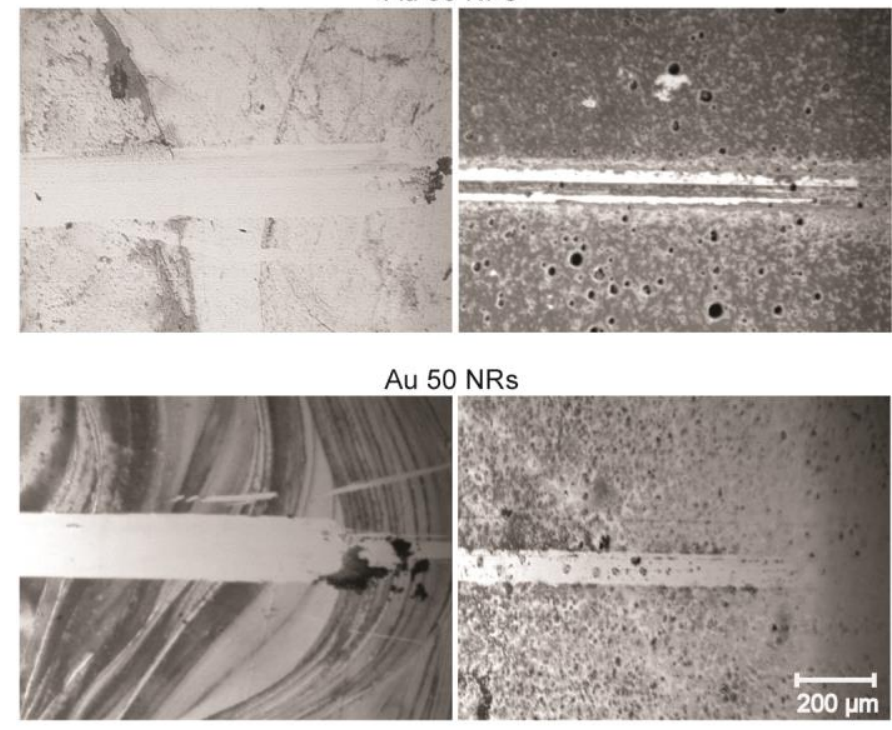

(a)

SEM micrographs of macroscale wear scars in dry conditions
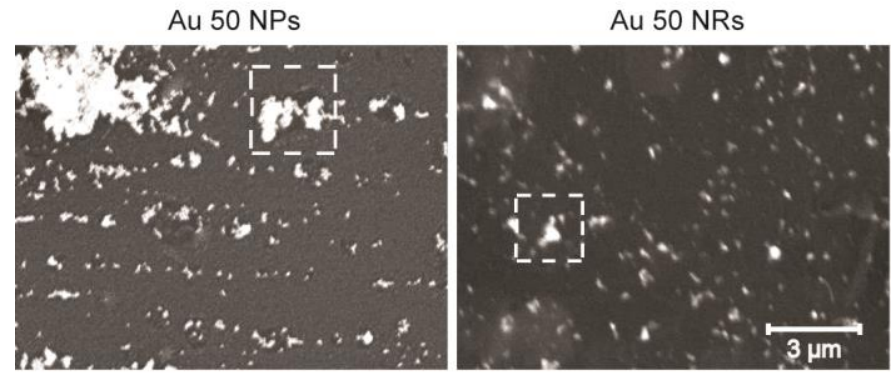

(b)

(Maharaj and Bhushan, 2014b)

Figure 19 
scars shown, are larger for sliding under dry environments compared to sliding under water environments, as the amount of wear is greater. A greater amount of wear is seen without the nano-objects in dry and water environments compared to cases where the NPs and NRs are added.

Figure 19b shows magnified SEM micrographs of wear scars for NPs and NRs in dry environments. Agglomerations of NPs and NRs within the squares can be observed. Agglomeration occurs during the wear process as nano-objects stick together due to attractive van der Waals and meniscus forces.

Figure 20 shows the coefficient of friction data for all wear cases for 500 cycles. The coefficient of friction is higher under dry environments, which corresponds to higher wear as seen in Figure 19a. The lowest coefficient of friction coincides with the cases of least wear. Compared to the nanoscale, the coefficients of friction are higher when sliding on the NRs despite higher contact pressures on the nanoscale. As reported earlier, on nanoscale, the contact pressure range was from $0.2-0.6 \mathrm{GPa}$ and for the macroscale, it was $0.15 \mathrm{GPa}$. Higher contact pressures generally lead to an increase in contact area and therefore greater friction forces. However, on the nanoscale due to the small radius of the sliding ball $(50 \mu \mathrm{m})$, this is not significant in relation to the macroscale. On the macroscale due to the large radius of the ball $(1.5 \mathrm{~mm})$ there is much greater number of NRs (6420) that make contact with the ball and the silicon substrate as compared to a single NR on the nanoscale. Because of this increased contact, the friction forces are greater resulting in larger coefficients of friction. 


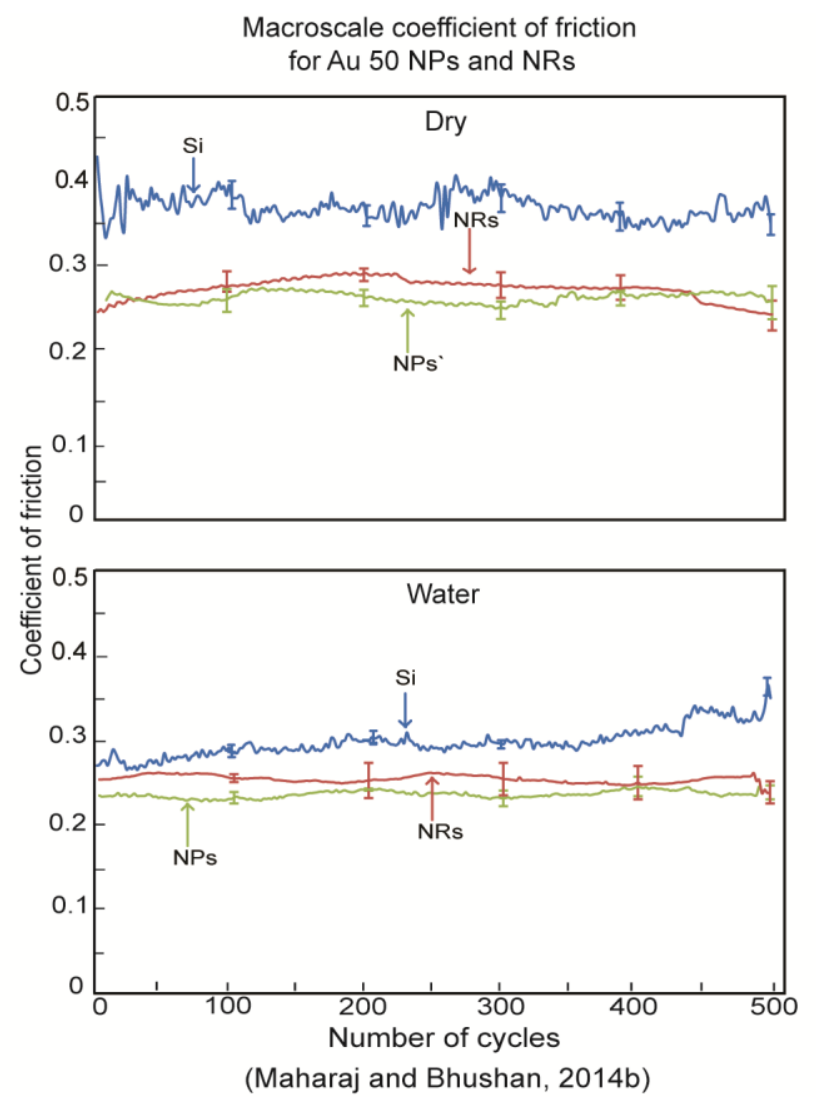

Figure 20: Macroscale coefficient of friction data after 500 cycles at a normal load of 200 $\mathrm{mN}$, in dry and water conditions with and without Au 50 NPs and NRs (Maharaj and Bhushan, 2014b). The lowest coefficient of friction occurs with $\mathrm{Au} 50$ NPs in water.

On the macroscale, the addition of NPs and NRs in general results in the lower coefficients of friction for all wear cases compared to the bare silicon substrate. The nano-objects create a barrier between the two surfaces and reduce the contact area. Studies by Tevet et al. (2011) suggested that under a higher contact pressure, using single inorganic fullerene (IF) $\mathrm{MoS}_{2}$ and $\mathrm{WS}_{2}$ nano-objects, sliding is more likely to take place 
and at lower pressures, rolling occurs. As discussed by Maharaj and Bhushan (2014b) a similar mechanism is proposed for the macroscale wear test. It is believed that the reduction in the friction coefficient is due to sliding on the deformed NPs and NRs, where deformation can occur when initial contact is made with larger nano-objects which are fewer in number, resulting in higher contact pressures. It is also expected that some nanoobjects will be dragged along with the sapphire ball as the number of cycles increases. As more nano-objects support the load the contact pressure is reduced. This increases the chance of rolling of the undeformed nano-objects as part of the friction reduction mechanism. Similar to multiple nano-object contact on the nanoscale, a greater probability of rolling is expected with the NPs compared to the NRs. These mechanisms are illustrated in Figure 16a-c and are believed to be responsible for the lower coefficient of friction. The coefficient of friction is lower for the NPs compared to the NRs due to the lower contact area and greater probability of rolling. However, the NRs still help to reduce the friction coefficient significantly compared to sliding on the bare substrate. In submerged in water environments the results are similar to the nanoscale, where the coefficients of friction are lower than in dry environments in general. This is due to the presence of a surface of low shear strength for sliding. Aside from the reduced contact area, there is a greater chance of rolling with the NPs than with the NRs, similar to dry environments which results in lower coefficients of friction compared to the NRs. 


\subsection{Summary}

$\mathrm{Au}$ NPs, CNHs and $\mathrm{MoS}_{2}$ and $\mathrm{WS}_{2}$ MWNTs were investigated for their effect on friction and wear in dry and liquid environments in water, dodecane and glycerol. Studies were conducted in both single and multiple nano-object contacts with the aid of an AFM. In macroscale studies, a ball on flat tribometer was used.

For single nano-object contact, the lowest friction forces occur with the CNHs compared to $\mathrm{Au}$ NPs and $\mathrm{MoS}_{2}$ and $\mathrm{WS}_{2} \mathrm{MWNT}$ s due to the reduced contact area provided by the roughness of the nanohorns and low meniscus force contribution in dry environments. Lower friction forces occur in submerged in liquid environments compared to dry environments due to elimination of meniscus force and sliding on a low shear strength surface among the various liquids. The highest friction forces occur in glycerol due to high viscosity.

For multiple nano-object contact, sliding over nano-objects reduced coefficients of friction compared to sliding on the bare silicon substrate due to reduced contact area and rolling and sliding among the various nano-objects. The lowest coefficient of friction occurs with the CNHs compared to the other nano-objects due to the mechanisms mentioned for single nano-object contact in dry environments. In nanoscale wear experiments in dry environments, the addition of nano-objects reduces the real area of contact and reduces the wear of the substrate through rolling and sliding of the nanoobjects.

In macroscale studies sliding over the various nano-objects reduced friction and wear due to the mechanisms mentioned in multiple nano-object contact on the nanoscale. 
Additionally, in dry environments, sliding on the MWNTs reduced the coefficient of friction and the wear due to exfoliation and tribofilm formation. The lowest coefficient of friction and wear in all cases occur with CNHs due to the reduced contact area. The coefficients of friction were also lower in submerged in liquid environments compared to dry condition due to mechanisms mentioned on the nanoscale. The more viscous liquids results in better wear protection and lower coefficient of friction due to their ability to support the load and not be easily squeezed out of the contact area. 


\title{
Chapter 5: Friction and wear in dry and liquid environments: Au NPs and NRs, CNHs and $\mathrm{MoS}_{2}$ and $\mathrm{WS}_{2}$ MWNTs
}

\author{
5.1 Introduction \\ In this section, results for experiments in single and multiple nano-object contact \\ are given for dry environments and submerged in liquid environments for water, \\ dodecane and glycerol. In single nano-object contact, the friction forces are presented for \\ Au 90 NPs, CNHs and $\mathrm{MoS}_{2}$ and $\mathrm{WS}_{2}$ MWNTs. For multiple nano-object contact, \\ coefficient of friction data are given and explained in detail. In addition, wear data for \\ both nanoscale and macroscale with and without addition of nanoparticles and nanotubes \\ are also presented. Morphological characterization of the nano-objects and wear scars are \\ also performed.
}

\subsection{Single nano-object contact}

\subsubsection{Nanoscale friction}

For single nano-object contact in dry environments, a sharp tip is used to manipulate or push the nano-objects laterally (Maharaj and Bhushan, 2012). For both Au 90 NPs and CNHs which are spherical in nature, the manipulation can involve rolling, sliding, and rotation about the vertical axis (Sitti, 2004). In the case of the $\mathrm{MoS}_{2}$ and $\mathrm{WS}_{2}$ 
MWNTs manipulation can involve rolling, sliding as well as in-plane rotation which occurs at a pivot point. This can happen when the nanotubes are not pushed directly at the center of its length. Similar observations were found for manipulations of carbon nanotubes on mica and graphite (Falvo et al., 1999).

Figure 21a presents the results for the average friction forces for Au 90 NPs, $\mathrm{CNHs}$ and $\mathrm{MoS}_{2}$ and $\mathrm{WS}_{2}$ MWNTs in dry and submerged in liquid environments. The friction force presented is the result of adhesion between the nano-objects and the silicon substrate. The adhesive force can include both van der Waals and meniscus forces for dry environments; however under submerged in liquid environments, meniscus forces are eliminated. There is an overall trend towards lower friction forces for the $\mathrm{CNHs}$ and $\mathrm{MoS}_{2}$ and $\mathrm{WS}_{2} \mathrm{MWNTs}$ compared to Au 90 NPs. In the case of the nanotubes it is not believed that exfoliation and tribofilm formation are responsible for the lower values as discussed by Maharaj et al. (2013) since there is no normal load acting on them.

The higher friction force observed for the Au 90 NPs is due to attractive (adhesive) meniscus forces from water vapor in the atmosphere that condenses around the contact area of the nanoparticles and silicon substrate. These attractive forces are larger on materials with higher work of adhesion $\left(W_{a}\right)$. Table A.3 (see Appendix A) gives the work of adhesion for thin films of $\mathrm{Au}, \mathrm{MoS}_{2}$ and $\mathrm{WS}_{2}$ and graphene with their corresponding contact angles. $W_{a}$ is calculated from the Dupré equation, given as:

$$
W_{a}=\gamma_{l}(1+\cos \theta)
$$

In this equation, $\gamma_{l}$ is the surface tension or free surface energy of a liquid, which is the additional energy available at the surface of the liquid due to fewer bonds with 
Figure 21: Friction force for $\mathrm{Au} 90 \mathrm{NPs}, \mathrm{CNHs}$ and $\mathrm{MoS}_{2}$ and $\mathrm{WS}_{2} \mathrm{MWNT}_{\mathrm{N}}$ on the silicon substrate during manipulation, at normal loads of (a) $300 \mathrm{nN}$ in air and (b) $1 \mathrm{nN}$ in liquids. The vertical scale is magnified for data in liquids. (c) Coefficients of friction for both dry and submerged in liquid environments, with and without nano-objects. Friction forces and coefficient of friction are lower in submerged in water environments (Maharaj et al., 2013). 
Friction force (During manipulation in air) Sharp tip $15 \mathrm{~nm}$

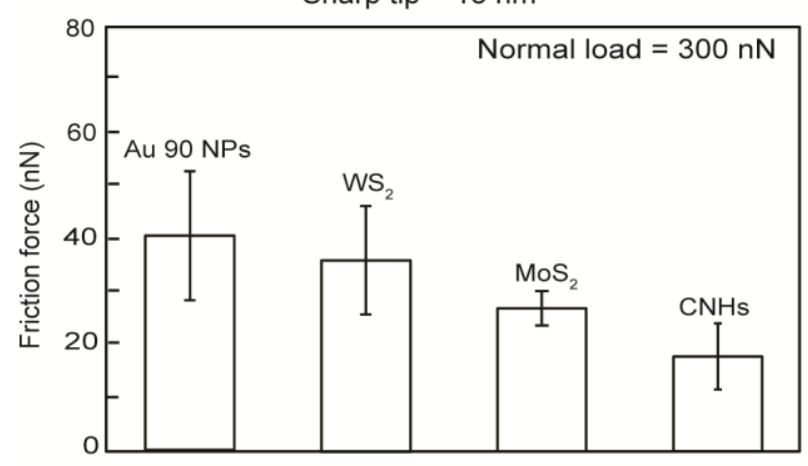

(a)

Friction force (During manipulation in liquid)



(b)

Coefficient of friction (During sliding on nano-objects)



(c)

(Maharaj et al., 2013)

Figure 21 
neighboring atoms and molecules (Bhushan, 2010). A value of $72 \mathrm{mN} / \mathrm{m}$ at $25{ }^{\circ} \mathrm{C}$ is used for water (Haynes, 2014) in this calculation. The static water contact angle, $\theta$, is the angle between the water droplet and the substrate surface (Bhushan, 2010). Higher work of adhesion means more work has to be done to separate the solid-liquid interface which contributes to greater attractive meniscus forces (Bhushan, 2010). The work of adhesion is highest with Au films and lowest with graphene films. It is expected that since Au 90 NPs will have a higher work of adhesion compared to CNHs and $\mathrm{MoS}_{2}$ and $\mathrm{WS}_{2}$ MWNTs, that the meniscus force contribution to adhesion will be greater. This increased adhesion accounts for the higher friction force observed with the Au 90 NPs. For the $\mathrm{CNHs}$, it is believed that the reduced contact area, due to the roughness provided by the nanohorns and the reduced meniscus force contribution, is responsible for the lowest observed friction forces.

Figure $21 \mathrm{~b}$ presents the friction forces for the nanoparticles and nanotubes submerged in liquid environments. The data for Au NPs only show friction force, in submerged in water environments as discussed in the experimental section. In liquids, the adhesive forces are due to van der Waals interactions since there are no meniscus bridges being formed in submerged in liquid environments. The lower friction forces in submerged in liquid environments compared to the dry environments can be attributed to the elimination of meniscus forces and nano-objects sliding on a low shear strength surface. Similar to dry environments, exfoliation of $\mathrm{MoS}_{2}$ and $\mathrm{WS}_{2}$ nanotubes does not occur and there is no contribution from tribofilm formation. The lowest friction forces 
occur with the CNHs due to the low contact area provided by the roughness of the nanohorns. The highest friction force occurs in glycerol, which has the highest viscosity of the liquids used and therefore the greatest contribution to viscous drag force. Even though dodecane has a greater viscosity than water, it is still not sufficiently high enough to give a significant contribution to drag forces on the nano-objects or the AFM tip, which explains the similarity in friction forces observed for both liquids with and without nano-objects.

\subsection{Multiple nano-object contact}

\subsubsection{Nanoscale friction and wear}

In multiple nano-object contact, the effect of normal load acting on the Au 90 NPs, CNHs and $\mathrm{MoS}_{2}$ and $\mathrm{WS}_{2}$ MWNTs between two surfaces is studied to determine the effects on friction and wear. Figure 21c summarizes the coefficient of friction for dry and submerged in liquid environments. Sliding in multiple nano-object contact results in lower friction forces in all conditions compared to sliding on the bare silicon substrate. In general, the friction forces were lower for sliding in submerged in liquid environments as compared to sliding in dry environments for nano-object coated and uncoated surfaces.

It has been demonstrated that sliding on multiple asperities on nanopatterned surfaces (Burton and Bhushan, 2005) results in friction reduction. In this particular case, the asperities are immobile, and reduction occurs as a result of the reduced contact area. For sliding on $\mathrm{Au} 90 \mathrm{NPs}, \mathrm{CNHs}$ and $\mathrm{MoS}_{2}$ and $\mathrm{WS}_{2}$ MWNTs, the friction force reduction can be attributed to the mobility of the nano-objects in addition to the reduced 
contact area. The resulting friction reduction mechanism can be attributed to the reduced contact area, the sliding over deformed nano-objects as well as the individual nanoparticles and nanotubes being dragged with the glass sphere as shown in Figure 16bc. It is also possible, as the glass sphere encounters a greater number of nanoparticles and nanotubes, the contact pressure is reduced allowing undeformed nano-objects to roll between the surfaces as shown in Figure 16a. Exfoliation and tribofilm formation as shown in Figure 16d-e are believed to be possible in dry environments for $\mathrm{MoS}_{2}$ and $\mathrm{WS}_{2}$ MWNTs as there is a general trend towards lower friction compared to Au 90. However this is not very significant due to the limited number of MWNTs under the $50 \mu \mathrm{m}$ sphere. In dry environments, the coefficient of friction data for the $\mathrm{CNHs}$ and the $\mathrm{MoS}_{2}$ and $\mathrm{WS}_{2}$ MWNTs are generally lower than the Au 90 NPs. As in the case of single nano-object contact, with the Au 90 NPs there is a higher meniscus force contribution to friction compared to the $\mathrm{CNHs}$ and $\mathrm{MoS}_{2}$ and $\mathrm{WS}_{2}$ MWNTs. With the CNHs the reduced contact area, as a result of the roughness of the nanohorns, and low meniscus force contribution, are responsible for the low friction forces.

In submerged in liquid environments the coefficients of friction were lower compared to dry. For water, as the meniscus forces are eliminated, the coefficient of friction data shows very little difference for Au $90 \mathrm{NPs}$ and $\mathrm{MoS}_{2}$ and $\mathrm{WS}_{2} \mathrm{MWNTs}$. The $\mathrm{CNHs}$ have the lowest coefficient of friction in all liquid environments due to the reduced contact area. The highest friction force and coefficient of friction occurs in glycerol, which has the highest viscosity and results in a greater contribution from viscous drag force similar to single nano-object contact followed by dodecane. The elimination of the 
meniscus forces, together with sliding on surface of low shear strength (Bhushan and Sundararajan, 1998), results in lower coefficients of friction.

For a potential lubricant to be considered effective, it must not only be able to reduce the coefficient of friction, but also protect the underlying surface. Figure 5.2 summarizes the wear data for sliding on $\mathrm{Si}$, and $\mathrm{Si}$ coated with $\mathrm{Au} 90$ and $\mathrm{CNH}$ and $\mathrm{MoS}_{2}$ and $\mathrm{WS}_{2} \mathrm{MWNTs}$, for 1,10 , and 100 cycles at a normal load of $20 \mu \mathrm{N}$ under dry environments. A $20 \mu \mathrm{m} \times 20 \mu \mathrm{m}$ area is imaged to show wear scars which are created over a $10 \mu \mathrm{m} \times 10 \mu \mathrm{m}$ area. Topography maps along with corresponding height profiles are also shown.

After 1 cycle the surface of the uncoated silicon appears rough during the early stages of wear as it becomes damaged. For the Au $90 \mathrm{NPs}, \mathrm{CNHs}$ and $\mathrm{MoS}_{2}$ and $\mathrm{WS}_{2}$ MWNTs coated surface, after 1 cycle the nano-objects are just beginning to be pushed out of the wear area. After 10 cycles the topography profile of the uncoated silicon surface appears smoother than after 1 cycle. This is due to polishing of the surface. The Au 90 NPs, CNHs and $\mathrm{MoS}_{2}$ and $\mathrm{WS}_{2}$ MWNTs are pushed out of the wear area and agglomerate around the edges of the wear scar. For the uncoated silicon substrate after 100 cycles a small amount of material is removed with a wear depth of approximately $0.25 \mathrm{~nm}$ as seen in the height profile. For the coated surfaces very few nano-objects remain in the wear area for $\mathrm{MoS}_{2}$ and WS 2 MWNTs whilst for CNHs and Au 90 NPs they are completely removed.

Figure 23 shows SEM micrographs of the wear scars in dry environments (First row) after 100 cycles. Magnified micrographs of the areas within the squares are shown 
Figure 22: Topography maps and 2-D profiles, at sections shown by the arrows, after sliding at 1, 10 and 100 cycles with a normal load of $20 \mu \mathrm{N}$ on Si and Si coated with Au 90 NPs, CNHs and $\mathrm{MoS}_{2}$ and WS 2 nanotubes (Maharaj et al., 2013). 
Wear- Glass sphere $50 \mu \mathrm{m}$
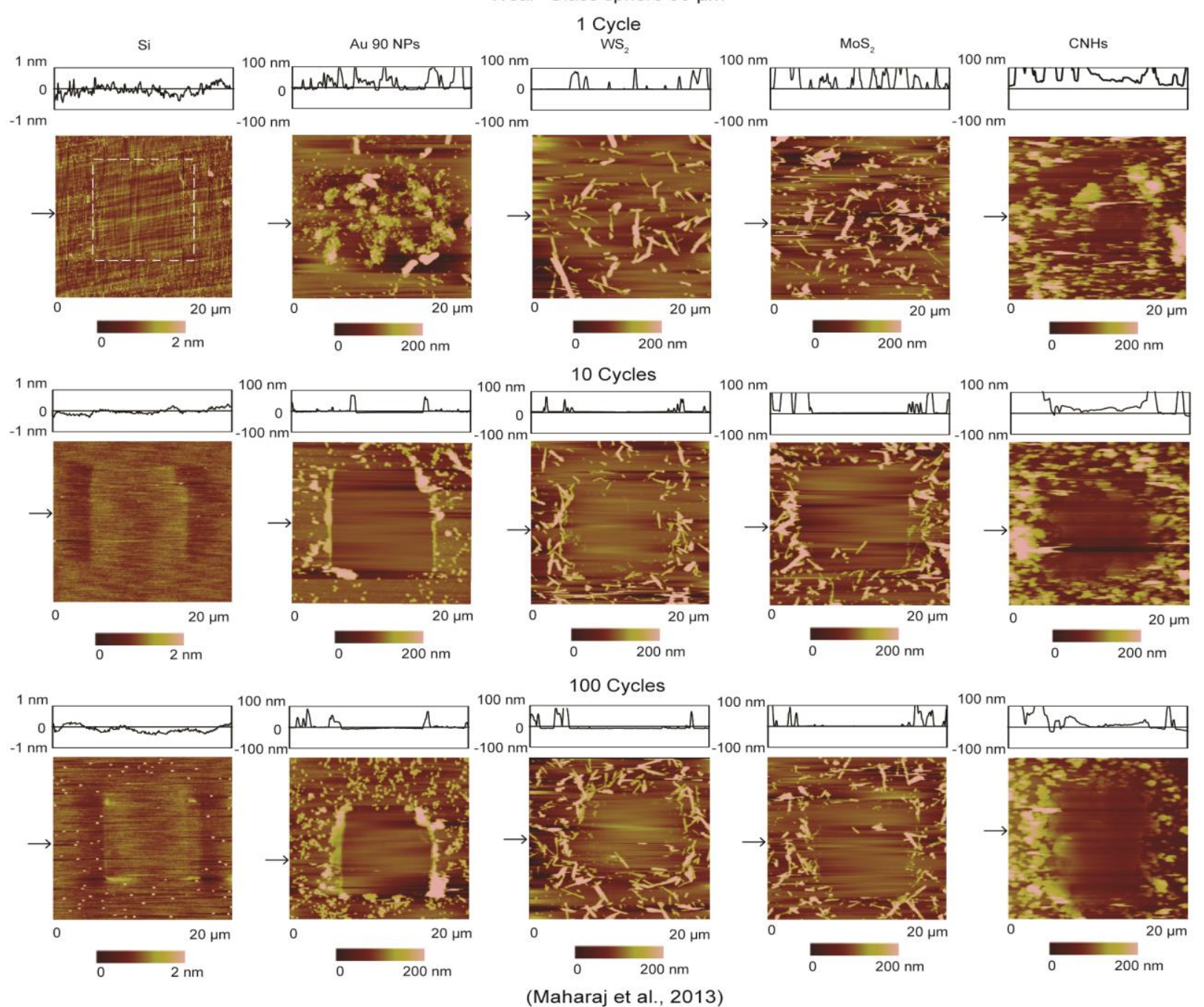

Figure 22 
SEM micrographs of nanoscale wear scars

in dry conditions after 100 cycles
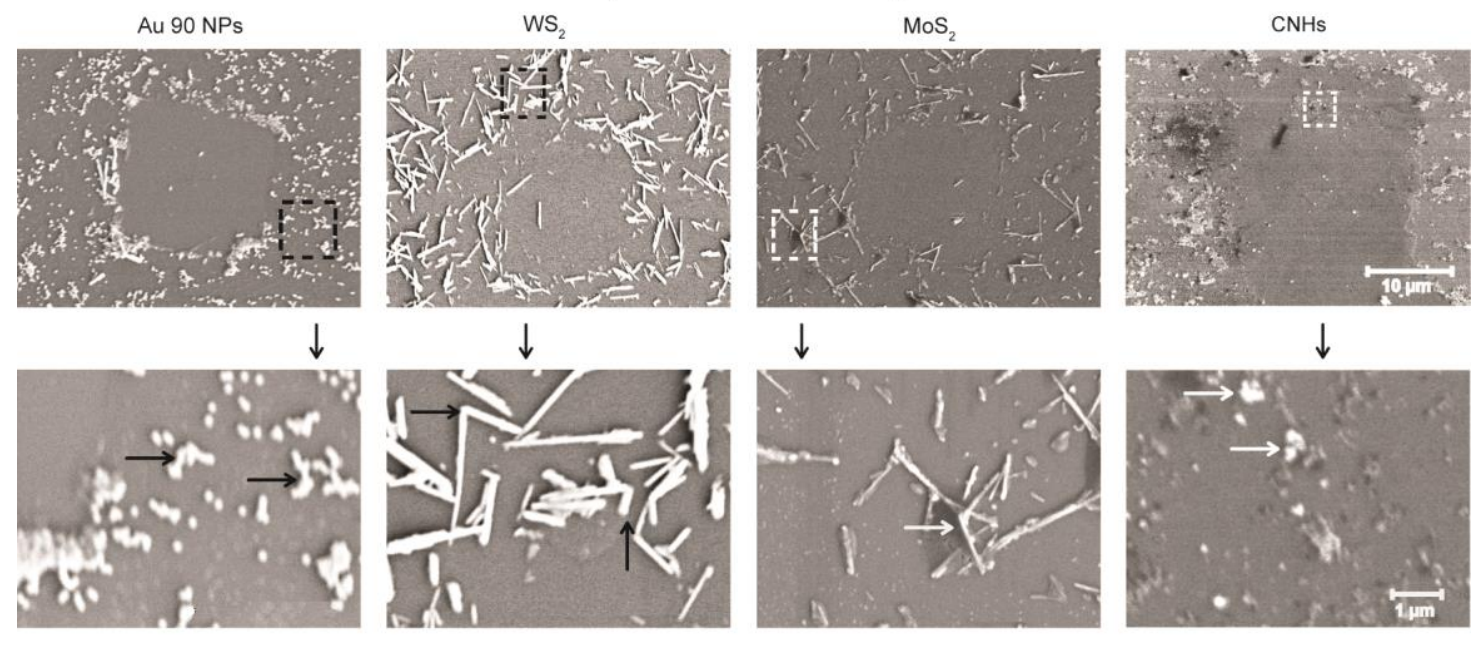

(Maharaj et al., 2013)

Figure 23: SEM micrographs of nanoscale wear scars for Au 90 NPs, CNHs and $\mathrm{MoS}_{2}$ and $\mathrm{WS}_{2}$ MWNTs in dry conditions after 100 cycles at a normal load of $20 \mu \mathrm{N}$ (First row). The nano-objects within the squares are magnified and the vertical arrows point to those micrographs in the second row. The magnified micrographs show agglomerated Au 90 NPs and CNHs and broken $\mathrm{MoS}_{2}$ and $\mathrm{WS}_{2} \mathrm{MWNTs}$ as indicated by arrows within the micrographs (Maharaj et al., 2013).

in the second row as indicated by the vertical arrows. In the magnified micrographs agglomerated $\mathrm{Au} 90 \mathrm{NPs}, \mathrm{CNHs}$ and broken $\mathrm{MoS}_{2}$ and $\mathrm{WS}_{2}$ MWNTs are pointed out by arrows within each micrograph. Agglomeration and breaking of the nano-objects can occur during the wear process. In the case of the $\mathrm{MoS}_{2}$ and $\mathrm{WS}_{2} \mathrm{MWNTs}$, broken nanotubes can also result while samples are being prepared during sonication as they collide with one another. 
It is believed, since the $\mathrm{Au} 90 \mathrm{NPs}, \mathrm{CNHs}$ and $\mathrm{MoS}_{2}$ and $\mathrm{WS}_{2} \mathrm{MWNT}$ remain in the wear area after 1 cycle, that the damage of the silicon surface would be less than that of an initially uncoated substrate. This occurs since the nano-objects are believed to roll and slide between the glass sphere and the substrate which promotes facile shearing of the two surfaces as shown in Figure 16 a-c, in addition to the reduced contact area provided by the nano-objects. For the $\mathrm{CNHs}$, the reduction in contact area due to the roughness provided by the nanohorns is expected to further reduce wear. After100 cycles, it is therefore expected that the greatest wear occurs on the bare silicon substrate and the presence of nanoparticles and nanotubes does provide protection of the substrate with the least wear occurring with the CNHs.

\subsubsection{Macroscale friction and wear}

In macroscale friction and wear tests a sapphire ball was slid over nanoparticle and nanotube coated and uncoated silicon substrates for 500 cycles with a stroke length of $10 \mathrm{~mm}$ under a normal load of $200 \mathrm{mN}$. Optical micrographs of wear scars in dry and liquid environments with and without nano-objects are displayed in Figure 24. In general, the widths of the wear scars shown, are larger for sliding in dry environments compared to sliding in liquid environments, as the amount of wear is greater. The addition of nanoobjects in dry and liquid environments reduces the amount of wear compared to cases without nano-objects.

In liquid environments, the wear scar widths decreases as the viscosity of the fluid increases with the greatest wear protection occurring in glycerol. This is due to the ability 
of the viscous fluid to support the normal load and not be squeezed out of the contact area.

Figure 25 shows magnified optical and SEM micrographs of the wear scars for all cases. In Figure 25a, nano-objects can be observed within the wear scars for all cases. The presence of the nano-objects after the wear test, further confirms that reduction in wear and size of the wear scars are due to addition of the nano-objects in dry and liquid environments. Figure 25b shows magnified SEM micrographs of wear scars for Au 90 NPs, CNH nanoparticles and $\mathrm{MoS}_{2}$ and $\mathrm{WS}_{2} \mathrm{MWNT}$ in dry environments. In the case of the Au 90 NPs and CNHs wear scar, agglomerations of nanoparticles within the squares can be observed. 
Optical micrographs of macroscale wear scars
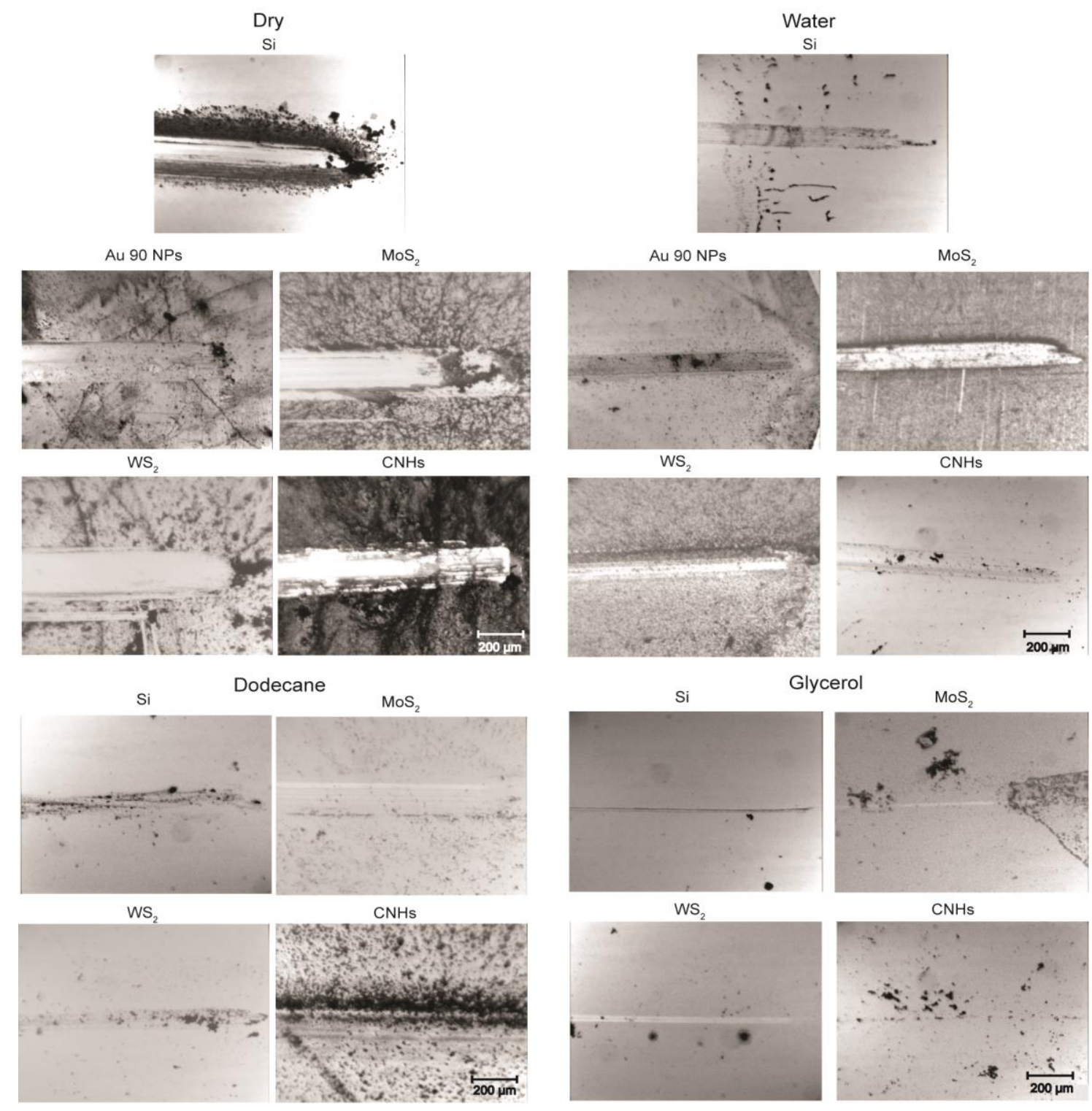

(Maharaj et al., 2013)

Figure 24: Optical micrographs of macroscale wear scars taken after 500 cycles at a normal load of $200 \mathrm{mN}$, for friction tests, in dry, water, dodecane and glycerol conditions with and without $\mathrm{Au} 90 \mathrm{NPs}, \mathrm{CNHs}$ and $\mathrm{MoS}_{2}$ and $\mathrm{WS}_{2}$ nanotubes (Maharaj et al., 2013). The lowest wear occurs in glycerol with CNHs 
Figure 25: Magnified (a) optical micrographs inside the wear scar on the macroscale taken after 500 cycles, at a normal load of $200 \mathrm{mN}$, in dry, water, dodecane and glycerol conditions with and without $\mathrm{Au} 90$ NPs, CNHs and $\mathrm{MoS}_{2}$ and $\mathrm{WS}_{2}$ MWNTs and (b) SEM micrographs of the wear scar for agglomerated Au 90 NPs, CNHs along with crushed or broken $\mathrm{MoS}_{2}$ and $\mathrm{WS}_{2}$ MWNTs in dry environments shown within the squares (Maharaj et al., 2013 Maharaj et al., 2013). 
Optical micrographs of macroscale wear scars (Images magnified)

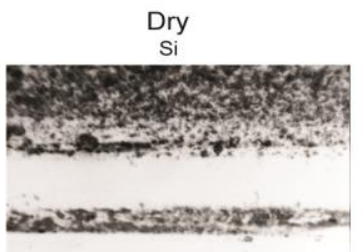

Au 90 NPs



$\mathrm{WS}_{2}$
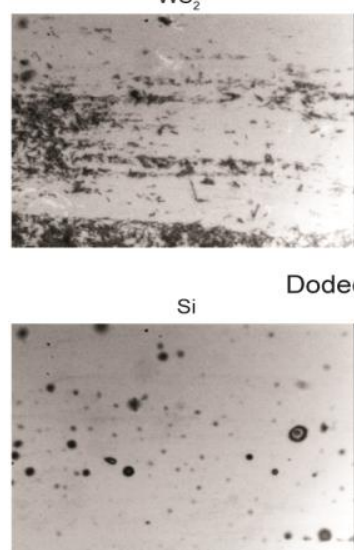

$\mathrm{WS}_{2}$

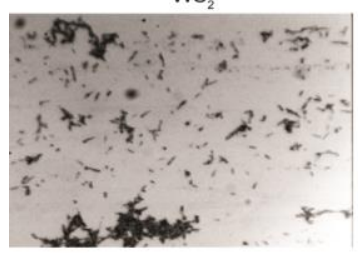

Au 90 NPs

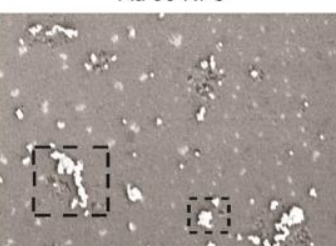

$\mathrm{CNH}$
$\mathrm{MoS}_{2}$

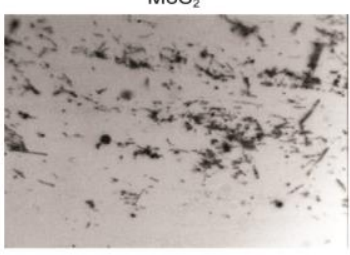

$\mathrm{CNHS}$

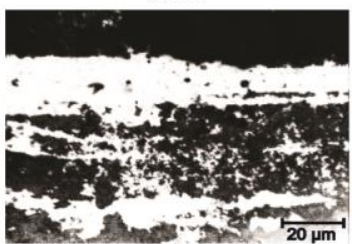

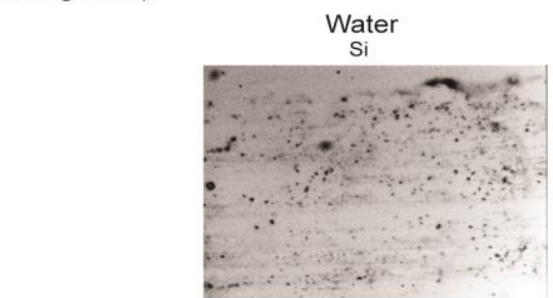


$\mathrm{WS}_{2}$
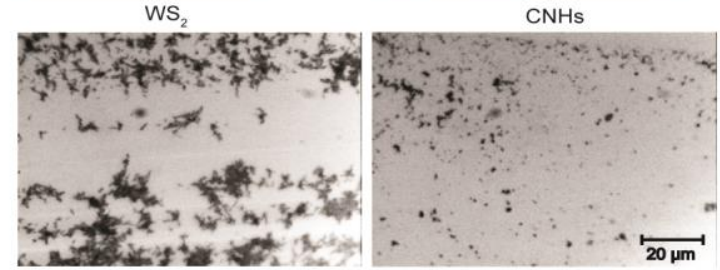

Glycerol


$\mathrm{WS}_{2}$
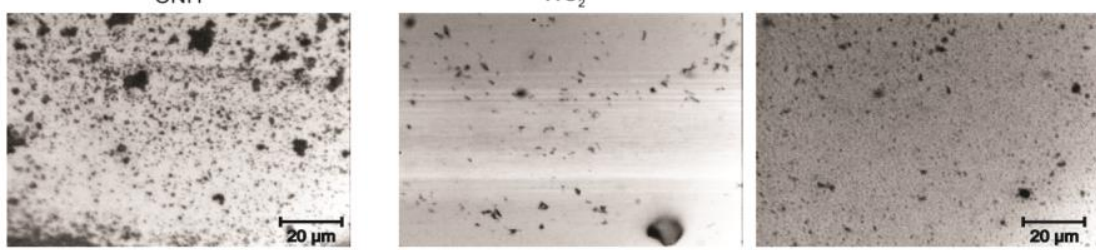

(a)

SEM micrographs of macroscale wear scars in dry conditions

$\mathrm{WS}_{2}$

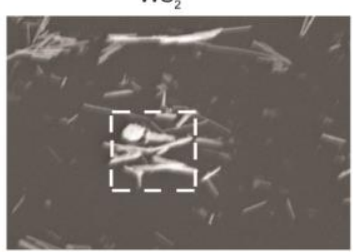

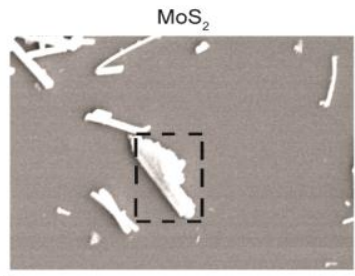

(b)

(Maharaj et al., 2013)

Figure 25 
Figure 26: Macroscale coefficient of friction data after 500 cycles at a normal load of 200 $\mathrm{mN}$, in dry, water, dodecane and glycerol envionments with and without $\mathrm{Au}$ 90 NPs, CNHs and $\mathrm{MoS}_{2}$ and $\mathrm{WS}_{2}$ MWNTs (Maharaj et al., 2013). The lowest wear occurs in glycerol with CNHs. 
Macroscale coefficient of friction
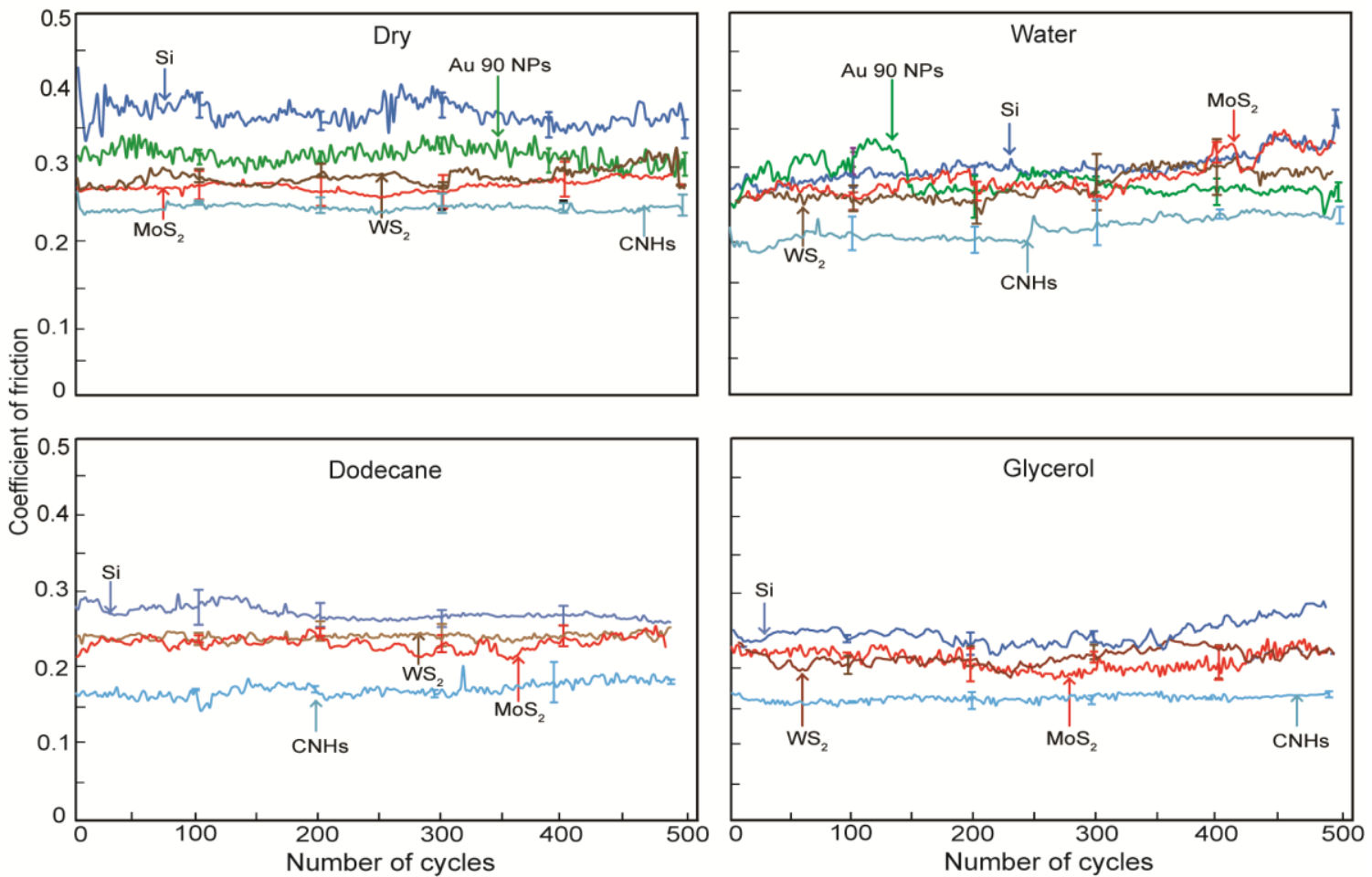

(Maharaj et al., 2013)

Figure 26 
Agglomeration occurs during the wear process as nanoparticles are pressed together and are attracted to each other due to van der Waals and attractive meniscus forces. For both $\mathrm{MoS}_{2}$ and $\mathrm{WS}_{2}$ wear scars, the SEM micrographs show crushed and broken nanotubes within the squares compared to those in Figure 10a-b, which depicts undeformed nanotubes. It is believed that in the process of being crushed, the outer layers of the nanotubes break apart leading to exfoliation of the nanotubes and tribofilm formation as discussed by Maharaj et al. (2013). Figure 26 shows the coefficient of friction data for all wear cases for 500 cycles.

Higher coefficients of friction, corresponds to greater wear, which occurs under dry environments. The lowest coefficients of friction coincide with the cases of least wear observed in Figure 25. The addition of nanoparticles and nanotubes in general results in the lower coefficients of friction for all wear cases compared to the bare silicon substrate. The Au 90 NPs, CNHs and $\mathrm{MoS}_{2}$ and WS 2 MWNTs create a barrier between the two surfaces and reduce the contact area. Tevet et al. (2011) suggested that under a higher contact pressure sliding is more likely to take place with single inorganic fullerene (IF) $\mathrm{MoS}_{2}$ and $\mathrm{WS}_{2}$ nano-objects and at lower pressures, rolling occurs. It is believed that the observed reduction in the friction coefficient is due to sliding on the deformed or crushed nanotubes, where deformation can occur when initial contact is made with larger nanoparticles and nanotubes which are fewer in number, resulting in higher contact pressures. It is also expected that some nano-objects will be dragged along with the sapphire ball as the number of cycles increases. As more nano-objects support the load the contact pressure is reduced. This increases the chance of rolling of the undeformed 
nano-objects as part of the friction reduction mechanism. These mechanisms are illustrated in Figure 16a-c and are believed to occur for the nano-objects.

Figure 16d-e illustrates the exfoliation of the outer layers of $\mathrm{MoS}_{2}$ and $\mathrm{WS}_{2}$ MWNTs and formation of an easily sheared tribofilm. This process is thought to be responsible for the lower friction coefficient observed with $\mathrm{MoS}_{2}$ and $\mathrm{WS}_{2} \mathrm{MWNTs}$ compared to Au 90 NPs in dry environments as discussed by Maharaj et al. (2013). It is believed that the reduced moisture and high contact pressures results in some exfoliation of the outer layers of the nanotubes. As sliding progresses, moisture becomes adsorbed onto the nanotubes, and with the presence of oxygen, oxidizes the $\mathrm{MoS}_{2}$ and $\mathrm{WS}_{2}$ MWNTs which results in an increase in friction due to formation $\mathrm{MoO}_{3}$ and $\mathrm{WO}_{3}$ (Pope et al., 1988; Prasad et al., 1993). The increase in the coefficient of friction occurs as the tribofilm formation is interrupted and the layers clump together and are no longer easily sheared. Since graphene is chemically inert (Kim et al., 2011), as sliding progresses there is no reaction with the moisture that is adsorbed onto the surface of the CNHs, which prevents deterioration of the nanoparticles. The lowest coefficient of friction is found on the CNHs coated surface. This is as a result of the low contact area due to the roughness provided by the nanohorns in addition to the mechanisms mentioned in the previous paragraph.

In liquid environments the results are similar to the nanoscale, where the coefficients of friction are lower than in dry environments in general. This is due to the presence of a surface of low shear strength for sliding. For CNHs submerged in water, the coefficient of friction is the lowest of all four nano-objects due to the reduced contact 
area provided by the roughness of the nanohorns. In the case of $\mathrm{MoS}_{2}$ and $\mathrm{WS}_{2} \mathrm{MWNTs}$, after 200 cycles the coefficient of friction rises and becomes higher than Au 90 NPs and CNHs and reaches that of bare silicon. Similar to dry environments the friction increase is due to the oxidation of the $\mathrm{MoS}_{2}$ and $\mathrm{WS}_{2} \mathrm{MWNTs}$. For both cases of sliding in dodecane and glycerol the coefficient of friction is lower sliding on a nano-object coated surface compared to the bare silicon. The coefficient of friction is lower for the CNHs compared to the MWNTs similar to the submerged in water case. After approximately 400 cycles for sliding on nanoparticles and nanotubes in dodecane, the coefficient of friction begins to rise as wear of the substrate takes place. In the case of glycerol there is also a rise in coefficient of friction for the nanotubes after 400 cycles, however the coefficient remains steady for the $\mathrm{CNH}$ nanoparticles. This is due to the combined effect of the reduced contact of $\mathrm{CNH}$ nanoparticles along with the ability of glycerol to better support the load due to its high viscosity which reduces the wear. The lowest coefficient of friction and least wear takes place in glycerol with CNHs.

\subsection{Summary}

Au NPs, CNHs and $\mathrm{MoS}_{2}$ and $\mathrm{WS}_{2}$ MWNTs were investigated for their effect on friction and wear in dry and liquid environments in water, dodecane and glycerol. Studies were conducted in both single and multiple nano-object contacts with the aid of an AFM. In macroscale studies, a ball on flat tribometer was used.

For single nano-object contact, the lowest friction forces occur with the CNHs compared to $\mathrm{Au}$ NPs and $\mathrm{MoS}_{2}$ and $\mathrm{WS}_{2} \mathrm{MWNT}$ sue to the reduced contact area 
provided by the roughness of the nanohorns and low meniscus force contribution in dry environments. Lower friction forces occur in submerged in liquid environments compared to dry environments due to elimination of meniscus force and sliding on a low shear strength surface among the various liquids. The highest friction forces occur in glycerol due to high viscosity.

For multiple nano-object contact, sliding over nano-objects reduced coefficients of friction compared to sliding on the bare silicon substrate due to reduced contact area and rolling and sliding among the various nano-objects. The lowest coefficient of friction occurs with the CNHs compared to the other nano-objects due to the mechanisms mentioned for single nano-object contact in dry environments. In nanoscale wear experiments in dry environments, the addition of nano-objects reduces the real area of contact and reduces the wear of the substrate through rolling and sliding of the nanoobjects.

In macroscale studies sliding over the various nano-objects reduced friction and wear due to the mechanisms mentioned in multiple nano-object contact on the nanoscale. Additionally, in dry environments, sliding on the MWNTs reduced the coefficient of friction and the wear due to exfoliation and tribofilm formation. The lowest coefficient of friction and wear in all cases occur with $\mathrm{CNHs}$ due to the reduced contact area. The coefficients of friction were also lower in submerged in liquid environments compared to dry condition due to mechanisms mentioned on the nanoscale. The more viscous liquids results in better wear protection and lower coefficient of friction due to their ability to support the load and not be easily squeezed out of the contact area 


\section{Chapter 6: Nanoscale friction: $\mathrm{MoS}_{2}$ and $\mathrm{WS}_{2}$ MWNTs}

\subsection{Introduction}

In this section, results from experiments on topography, scale, orientation and material effects on friction for $\mathrm{MoS}_{2}$ and $\mathrm{WS}_{2}$ MWNTs are presented. The various mechanisms for each are explained.

\subsection{Effects of topography}

Figure 27 shows corresponding topography and friction force maps for both $\mathrm{MoS}_{2}$ and $\mathrm{WS}_{2}$ MWNTs over a $2 \mu \mathrm{m} \times 2 \mu \mathrm{m}$ scan area (Top). The areas highlighted within the dashed squares $(500 \mathrm{~nm} \times 500 \mathrm{~nm})$ were imaged and area shown in the bottom row.

From the magnified images it can be observed that the features seen in the topography maps correspond to changes in the friction maps. The lighter regions on the friction maps correspond to higher friction and darker regions, lower friction (Maharaj and Bhushan, 2015a). These changes in friction occur where there are changes in the slope of the topography map as a result of the ratchet mechanism (Makinson, 1948; Bowden and Tabor, 1950; Ruan and Bhushan, 1994c; Sundararajan and Bhushan, 2000). As the AFM tip is scanned up a slope it experiences a higher lateral force and the friction 
force is a combination of the material friction and the slope of the asperity. Conversely, the friction orce is lower when descending as the slope term is subtracted out. These variations in friction do not occur due to the nanotube curvature as the change in the slope of the tube is negligible compared to the features on the tube. Sundararajan and Bhushan (2000) found that there is also a contribution to friction due to the collision of the tip when ascending a slope. This produces an additional torsion to the tip and contributes to the friction force being higher than when descending the slope. The magnitude of this force is dependent on the applied normal load and scanning velocity. The "collision" force is not present when descending the slope and only the ratchet mechanism is at work. These effects can be minimized by scanning on a flat surface where slope and "collision" contributions would be negligible which allows for isolating the material induced effects of friction. 
Topography and friction maps of $\mathrm{MoS}_{2}$ and WS 2 MWNTs

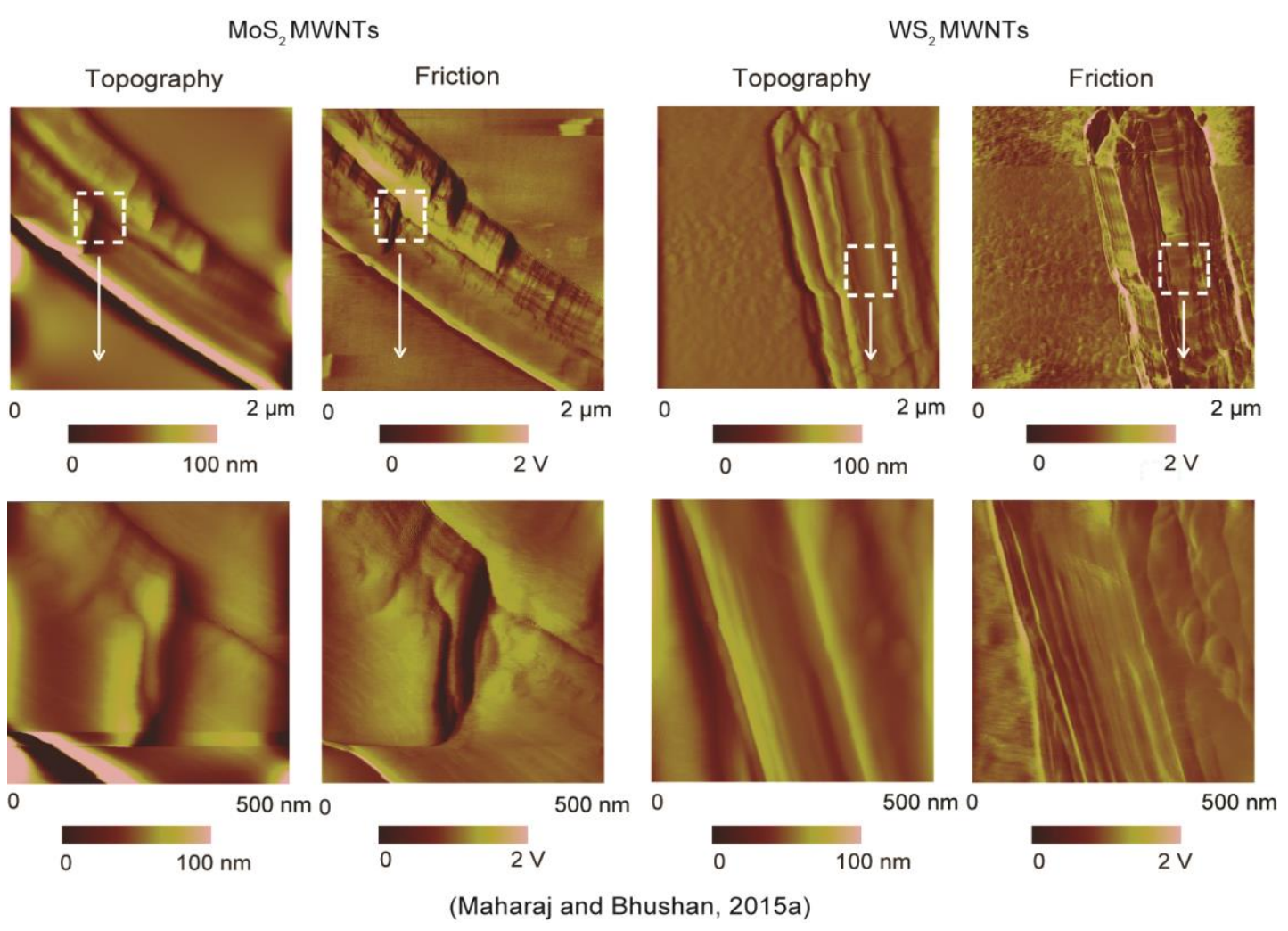

Figure 27: Examples of topography and friction maps of $\mathrm{MoS}_{2}$ (Left) and $\mathrm{WS}_{2}$ nanotubes (Right) with arrows pointing to magnified images of the areas highlighted by dashed squares (Maharaj and Bhushan, 2015a). 


\subsection{Effects of scale, orientation and material}

Figure 28 shows representative topography and friction force maps for $\mathrm{MoS}_{2}$ (Top) and $\mathrm{WS}_{2}$ MWNTs (Bottom) taken over a $5 \mathrm{~nm} \times 5 \mathrm{~nm}$ area to minimize roughness effects, as the surface asperities at this scale are expected to be smaller than the tip radius. This allows for the elimination of topographical features that may lead to variations in friction due to the ratchet and collision mechanism. For $\mathrm{MoS}_{2}$ the root mean squared roughness $(\mathrm{RMS})=0.16 \mathrm{~nm}$ and the peak to valley $(\mathrm{P}-\mathrm{V})$ roughness $=1.2 \mathrm{~nm}$ and for $\mathrm{WS}_{2}, \mathrm{RMS}=0.12 \mathrm{~nm}$ and $\mathrm{P}-\mathrm{V}$ roughness $=1 \mathrm{~nm}$. The coefficient of friction data was obtained within these areas.

Figure 29 shows coefficient of friction of data for the nanotubes at orientations of $0^{\circ}$ and $90^{\circ}$. The coefficient of friction of both nanotubes was found to be approximately 0.017. It was found that that coeffcient of friction was similar for both $\mathrm{MoS}_{2}$ and $\mathrm{WS} \mathrm{S}_{2}$ nanotubes since they are structurally similar. The coefficients of friction are lower than for sliding on silicon (0.05) due to shearing of the weakly bonded layers of the $\mathrm{MoS}_{2}$ and $\mathrm{WS}_{2}$ nanotubes and transfer of material on the AFM tip, which also leads to easier shearing. No significant orientation effects were observed (Maharaj and Bhushan, 2015a). Even lower coefficients of friction (ultra-low) have been reported by Ruan and Bhushan (1994a) on highly oriented pyrolytic graphite (0.006), Bhushan et al. (2008) on carbon nanotube, and Miura and Kamiya (2002) on $\mathrm{MoS}_{2}$ (0.003). For Ruan and Bhushan, (1994a) the ultra-low friction was observed in the [0001] plane. In the case of Miura and Kamiya (2002) ultra-low friction was observed as an AFM tip pressed onto a 


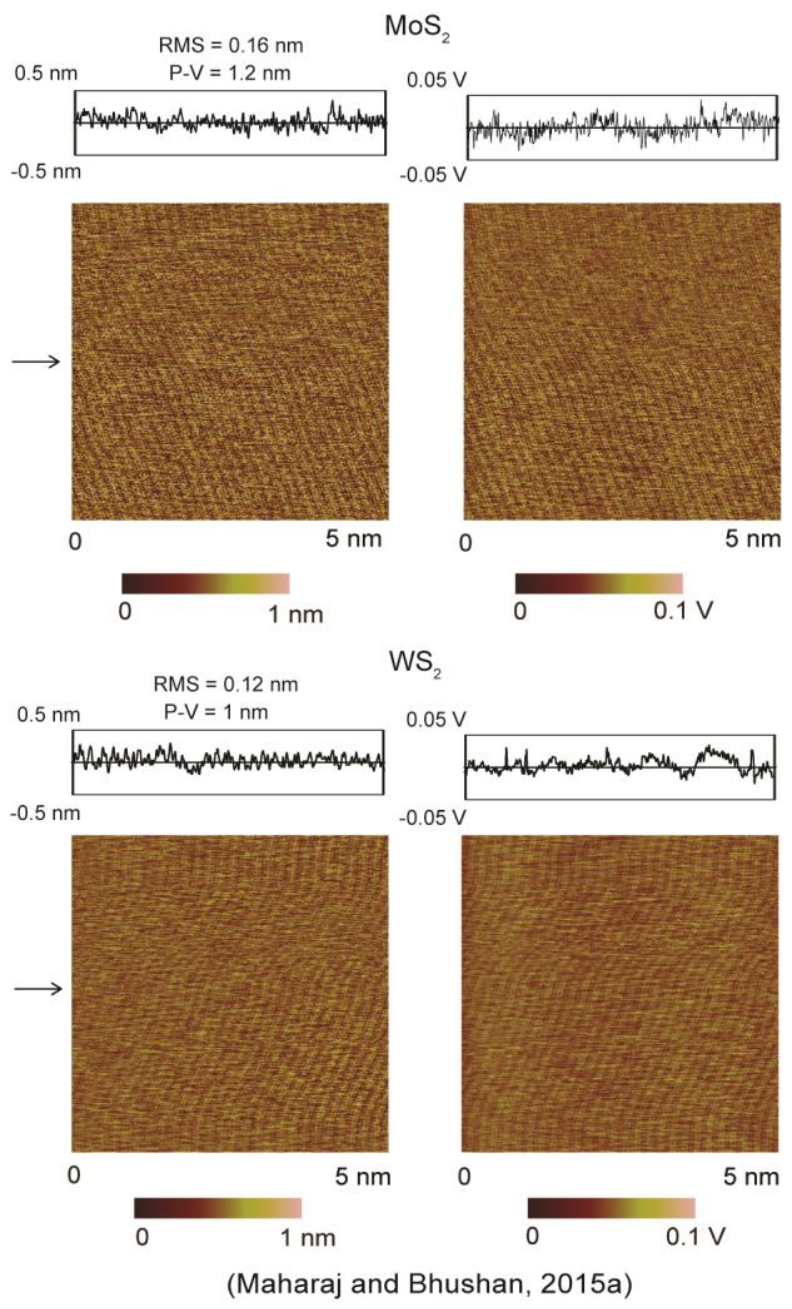

Figure 28: Examples of topography and friction maps of $\mathrm{MoS}_{2}$ (Top) and $\mathrm{WS}_{2} \mathrm{MWNTs}$ (Bottom) with arrows pointing to the sections on which the corresponding profiles were taken. For $\mathrm{MoS}_{2}, \mathrm{RMS}=0.16 \mathrm{~nm}$ and $\mathrm{P}-\mathrm{V}=1.2 \mathrm{~nm}$ and for $\mathrm{WS}_{2}, \mathrm{RMS}=0.12 \mathrm{~nm}$ and P-V $=1 \mathrm{~nm}$. (Maharaj and Bhushan, 2015a). 


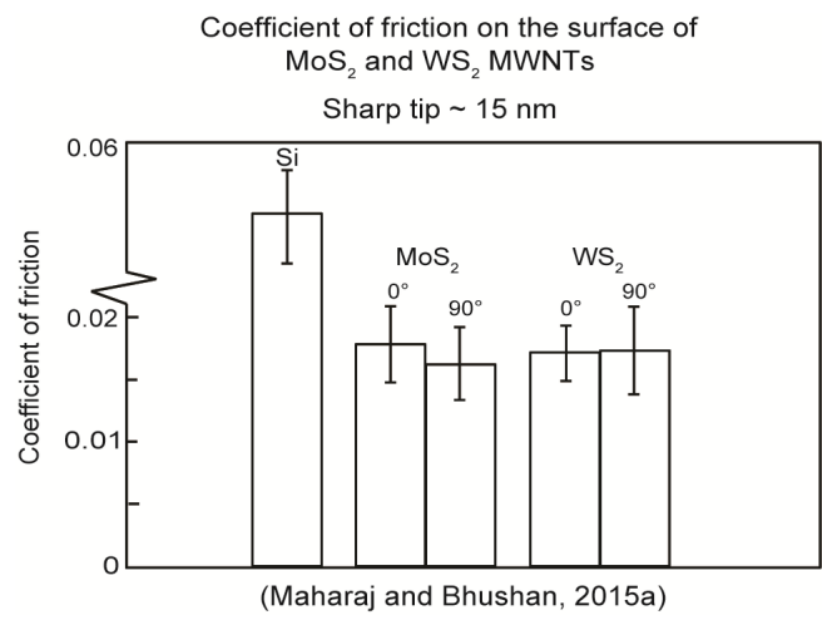

Figure 29: Coefficient of friction data for sliding on $\mathrm{MoS}_{2}$ and $\mathrm{WS}_{2} \mathrm{MWNTs}$ at nanotube orientations of $0^{\circ}$ and $90^{\circ}$ (Maharaj and Bhushan, 2015a). Material transfer from the MWNTs to the tip lowers friction compared to sliding on the substrate.

$\mathrm{MoS}_{2}$ flake, $1 \mathrm{~mm}$ square and several micrometers thick, was slid against a $\mathrm{MoS}_{2}$ substrate in the [10 $\overline{1} 0]$ direction of the substrate. Miura and Kamiya (2002) also found that when the angle of sliding is changed from this orientation, the coefficient of friction can be as high as 0.02. In the case of sliding on the $\mathrm{MoS}_{2}$ and $\mathrm{WS}_{2} \mathrm{MWNTs}$, there are several factors to consider that may mask this effect. In the case of the $\mathrm{MoS}_{2}$ substrate and flake, both were freshly cleaved from a natural $\mathrm{MoS}_{2}$ block and were exposed to ambient air during the experiment. The nanotubes, in addition to being studied in ambient air, were also stored in an environment with a relative humidity of approximately $50 \%$. Prolonged storage in such environments can lead to oxidization of both $\mathrm{MoS}_{2}$ and $\mathrm{WS}_{2}$ surfaces to $\mathrm{MoO}_{3}$ and $\mathrm{WO}_{3}$ respectively. Water vapor present can also condense in the 
defects of the crystal structure as shown in Figure 10 and would interrupt the easy shear of the basal planes (Pope and Pannitz, 1988; Uemura et al., 1990; Prasad and Zabinski, 1993). The combination of both effects is expected to alter the structure of both $\mathrm{MoS}_{2}$ and $\mathrm{WS}_{2}$ surfaces in such a way as to prevent any changes in friction due to orientation and observation of ultra-low friction (Maharaj and Bhushan, 2015a).

Macroscale friction for bulk $\mathrm{MoS}_{2}$ and $\mathrm{WS}_{2}$ have been reported to be on the order of 0.05 (Bhushan and Gupta, 1991) which is larger than the data reported here. Such scale effects on friction have been reported in various publications (Ruan and Bhushan, 1994c; Bhushan, 2011)

\subsection{Summary}

$\mathrm{MoS}_{2}$ and $\mathrm{WS}_{2}$ nanotubes were investigated to understand the contributions to friction mechanism as a result of topography and material induced effects. Sliding over sections of both nanotubes showed correlations between changes in topography and changes in friction force. This is attributed to the ratchet mechanism and the "collision" effect present when sliding up a slope. Sliding on flat sections of the nanotubes showed lower coefficients of friction compared to silicon due to the presence of transfer material on the AFM tip which facilitates shearing. No orientation effects were found. The coefficient of friction is believed be influenced by the presence of moisture and formation of oxide layers on the nanotubes which may mask orientation effects. Scale effects on friction are observed. Understanding the various contributions to friction is important for deciding the suitability of nano-objects for different applications. 


\section{Chapter 7: Mechanical behavior: Au thin film, NPs and NRs}

\subsection{Introduction}

In this section, first, hardness, creep and strain rate data are shown for a thin $\mathrm{Au}$ film (100 nm). Next, results for nanoindentation using a Berkovich tip for Au 500 NPs and compression using a flat punch are given for $\mathrm{Au} 500 \mathrm{NPs}, \mathrm{Au} 50$ NPs and $\mathrm{Au} 50$ NRs. For both deformation methods, representative load-displacement curves are presented. Morphological characterization, before and after deformation, is also shown. Finally load-displacement curves for repeat compression tests using the flat punch are presented.

\subsection{Nanoindentation}

\subsubsection{Thin film}

Indentation with a Berkovich tip was used to determine the mechanical properties and investigate creep and strain rate behavior of a $100 \mathrm{~nm}$ thick Au film as shown in Figure 30a-c.

In Figure 30a the mechanical properties for the thin film are illustrated by the bold diamond and squares, while the open diamonds and squares represent the properties of the bulk material. The hardness and Young's modulus for the thin film were found at maximum loads of 20, 4060 , and $80 \mu \mathrm{N}$. The Young's modulus, as a function of contact 
Figure 30: (a) Mechanical properties of thin films with hardness and Young's modulus as a function of contact depth represented by bold square and diamond datum points respectively with corresponding open diamond and square datum points representing bulk mechanical properties. Typical load displacement curves showing (b) creep at maximum loads of 40 and $80 \mu \mathrm{N}$ with a hold period of $200 \mathrm{~s}$, (c) effects of varying strain rate at a maximum load of $80 \mu \mathrm{N}$ for loading-unloading periods of 20 and $200 \mathrm{~s}$ (Maharaj and Bhushan, 2014a). 


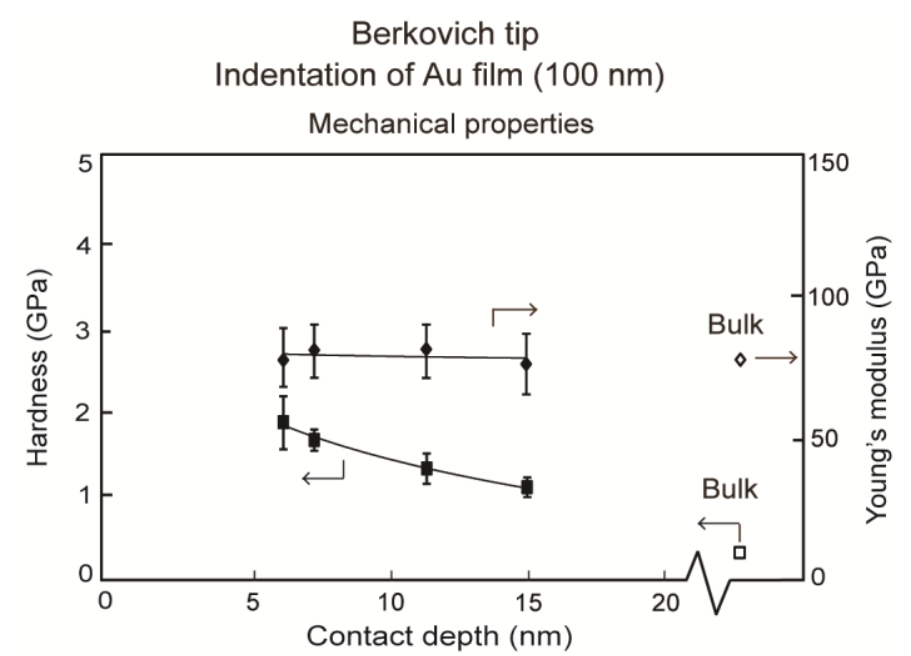

(a)

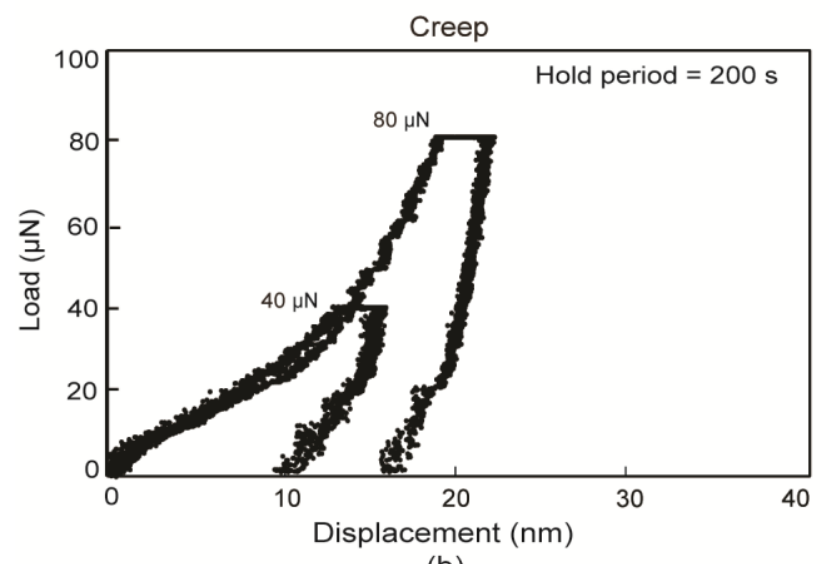

(b)



(c)

(Maharaj and Bhushan, 2014a)

Figure 30 
depth, is constant for the thin film and similar to that of bulk with little variation. The thin film hardness is greater than that of bulk and is not believed to be due to the hardness of the substrate (Maharaj and Bhushan, 2014a). It is generally accepted that the substrate affects the hardness if the depth of penetration is greater than 30\% (Bhushan and $\mathrm{Li}$, 2003) and this limit is not exceeded. Figure 30a shows an ISE where the hardness is greater for shallower penetration depths. This effect was also observed by Bhushan et al. (1996) and other researchers as outlined in Table A.1 (see Appendix A). A detailed analysis of the phenomenon was performed by Nix and Gao (1998) and explained. As discussed in the mechanism section, the GND's along with dislocations which are formed in the absence of strain gradients, known as statistically stored dislocations (SSD), hinder the formation and movement of new dislocations (Ashby, 1970; Fleck and Hutchinson, 1993; Nix and Gao, 1998). This results in a hardening effect and accounts for the observed increase in hardness at shallower contact depths for the Au film. The higher hardness compared to bulk also has a contribution from the Hall-Petch effect. In this case an increase in yield stress or hardening is observed compared to the macroscale due to the smaller grain sizes inherent in the thin film compared to bulk. The grain diameters in the representative image for the film shown in Figure 9 a were found to be $40 \mathrm{~nm} \pm 9 \mathrm{~nm}$. The hardening can occur through dislocation density mechanism and pile-up mechanism outlined and is illustrated in Figure 6a-b. However, due to the submicron thickness of the film, the nanosized grains limit the number of dislocation pile-ups. It is believed the dislocation density mechanism is primarily responsible for the higher hardness (Maharaj and Bhushan, 2014a). 
Figure 30b shows creep for typical load-displacement curves with maximum loads of 40 and $80 \mu \mathrm{N}$ with a hold period of 200 s. Figure 30c shows indentationdisplacement curves for different strain rates with a maximum load of $80 \mu \mathrm{N}$ for loading and unloading times of 20 and $200 \mathrm{~s}$. As mentioned in the experimental section, the creep and strain rate data gives an indication of whether the experiments are sensitive to hold times and loading and unloading rates. For the creep data there is there is very little difference in the displacement during the holding times at a load of $40 \mu \mathrm{N}$ compared to $80 \mu \mathrm{N}$. The strain rate data also shows a small amount of displacement from a loading and unloading time of 20-200 s. Due to this small change, the overall displacement seen in the load-displacement curves is still less than $30 \%$ of the film thickness and there are little to no substrate effects on the hardness data. Both experiments were performed at room temperature. Since Au is inert and does not form an oxide layer it is not believed that the increased displacement at longer holding and loading times is caused by a contaminant layer. According to analysis of experimental data from several research groups, (Li et al., 1991) creep can occur in most materials, even at room temperature. In materials with small grain sizes $(<0.3-0.4 \mu \mathrm{m})$ indentation creep is dominated by grain boundary (cobble) diffusive creep, which occurs by addition or removal of atoms from the boundary between two grains (Stevens, 1971; Li et al., 1991). This diffusional creep is believed to be responsible for increased displacements observed for creep and strain rate data. The low sensitivity of Au film to creep and strain rate is useful in tribological applications on the nanoscale in situations where constant load or low loading rates occur. This prevents the film from deforming easily. 
Similar experiments were not performed on the Au 500 NPs since over longer hold and loading and unloading times, the possibility of nanoparticles rotating and sliding is increased and results in the nanoparticles slipping out from under the indenter.

\subsubsection{Au NPs}

For probing of mechanical properties of the polycrystalline Au 500 NPs, indentation was performed at a maximum load of $80 \mu \mathrm{N}$. Figure 31a-b shows a typical load-displacement curve for indents at a maximum load of $80 \mu \mathrm{N}$, with topography maps of the nanoparticles over a $10 \mu \mathrm{m} \times 10 \mu \mathrm{m}$ scan area and 2-D profiles before and after indentation. The topography maps of the nanoparticles appear not totally circular due to tip shape convolution effects, however they are used to confirm the indentation of the nanoparticle and that the nanoparticle did not slip during loading and unloading. The vertical arrows on the load-displacement curves point to pop-in events during indentation. The horizontal white arrows indicate the nanoparticle of interest along with the section on which the profiles were taken. The pop-in events correspond to generation of new dislocations and multiplication of existing dislocations within the grain boundaries, which leads to an increase in displacement at a constant load. The eventual hardening is due to the dislocation density mechanism and pile up mechanism. This process repeats and results in subsequent slip and generation and multiplication of dislocations in neighboring grains (Gerberich et al., 2003; Yang and Vehoff, 2005; Faghihi and Voyiadajis, 2012; Maharaj and Bhushan, 2014a) resulting in further pop-ins. 
Figure 31: (a) Typical load displacement indentation curve at a maximum load of $80 \mu \mathrm{N}$ with vertical arrows showing pop-in events and (b) topography maps and 2-D profiles at sections shown by the horizontal arrows before indentation and after indentation. (c) Mechanical properties of Au 500 NPs with hardness and Young's modulus as a function of contact depth represented by bold square and diamond datum points respectively with corresponding open diamond and square datum points representing bulk mechanical properties (Maharaj and Bhushan, 2014a). 
Berkovich tip

Indentation of Au 500 NPs

Typical load-displacement curve showing pop-in events at $80 \mu \mathrm{N}$ as an example

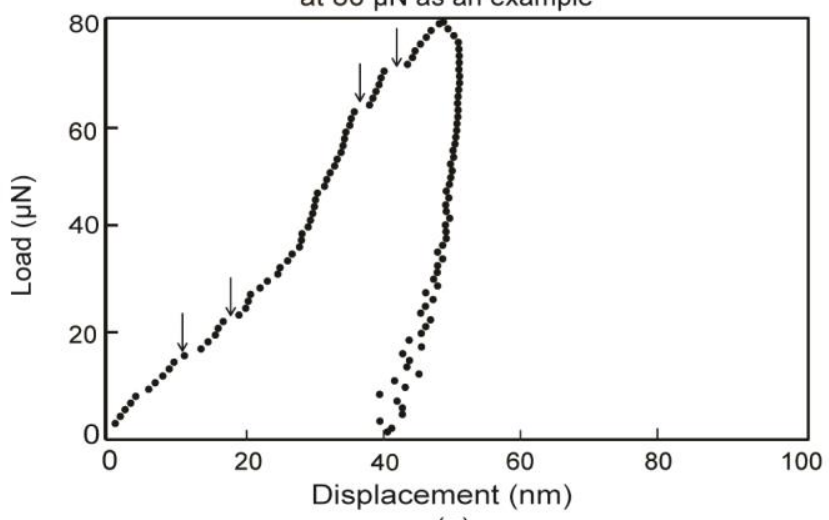

(a)


(b)

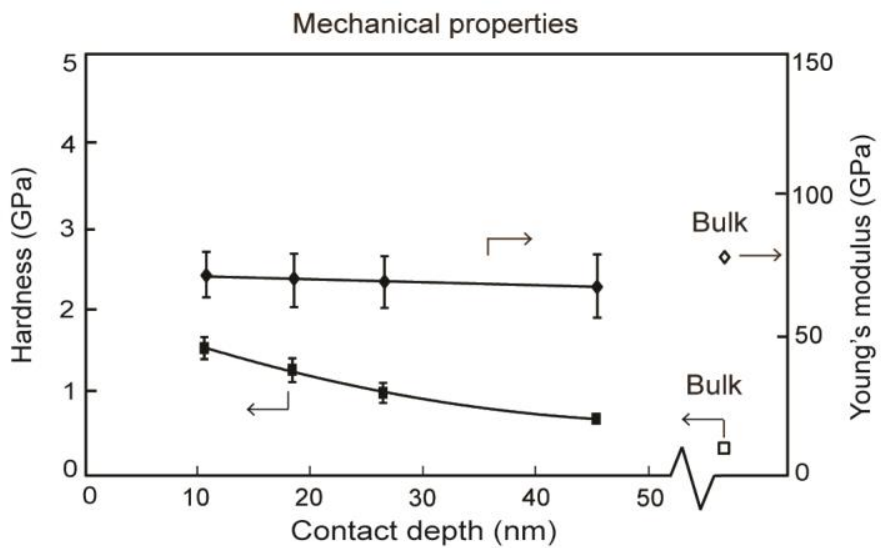

(c)

(Maharaj and Bhushan, 2014a)

Figure 31 
Figure 31c shows the mechanical properties for the Au 500 NPs as illustrated by the bold diamond and squares, while the open diamonds and squares represent the properties of the bulk material. The hardness and Young's modulus for nanoparticles were found at maximum loads of 20,40 60, and $80 \mu \mathrm{N}$. The Young's modulus, as a function of contact depth, is constant for the nanoparticles and slightly lower than that of bulk with little variation. An ISE for the hardness is also observed, similar to the thin film due to higher strain gradients at shallower depths. The hardness is also higher for the nanoparticles compared to bulk Au. This follows the 'smaller is stronger' phenomenon. The average grain diameter for the nanoparticle as shown in Figure 9b was found to be 96 $\mathrm{nm} \pm 30 \mathrm{~nm}$. It is believed that the nanometer sized grains are responsible for enhanced hardness. This is based on the mechanisms described in the Hall-Petch effect, where the dislocation density mechanism and the pile-up mechanism contributes to increased hardness. The decreasing grain sizes leads to higher yield stress and results in an increased hardness as observed.

Table A.4 (see Appendix A) presents data for hardness and Young's modulus of elasticity, in addition to the contact depth during indentation at a representative load of 80 $\mu \mathrm{N}$ for the Au nanoparticles and film. The hardness of the nanoparticles was found to be lower than the thin film. This is expected since the film has smaller grains with diameters of $40 \pm 9 \mathrm{~nm}$ compared to $96 \pm 30 \mathrm{~nm}$ for Au 500 NPs and higher resistance to yield. Both scales (100 nm and $500 \mathrm{~nm}$ ) show a higher hardness compared to bulk. This expands the possible uses of nanoscale Au in harsh environments where resistance to 
deformation under loading is important for reduced friction and wear by reducing contact area (Maharaj and Bhushan, 2014a).

\subsection{Compression: Au NPs and NRs}

Au 500 NPs, Au 50 NPs and Au 50 NRs were compressed to examine the differences between local deformation (nanoindentation) and global deformation (compression) and mechanical behavior during compression. Compression tests, similar to indentation tests, also simulate the types of contacts nanoparticles encounter during different friction and wear conditions. For this purpose, a tip approximately $3.5 \mu \mathrm{m}$ in radius was used to carry out compression tests.

For Au 500 NPs, Figure 32 shows a typical load displacement curve for compression at a maximum load of $80 \mu \mathrm{N}$, along with topography maps of the nanoparticles over a $10 \mu \mathrm{m} \times 10 \mu \mathrm{m}$ scan area and 2-D profiles before and after compression. The topography maps of the nanoparticles do not appear completely circular due to tip shape convolution effects, however they are used to confirm the compression of the nanoparticles. The vertical arrows point to pop-in events during indentation. In Figure 32b the horizontal white arrows indicate the nanoparticle of interest along with the section on which the profiles were taken. Pop-in events due to dislocations were observed as with nanoindentation. These occur in the latter half of the loading curve unlike with indentation, which shows pop-ins throughout the loading curve. Larger contact area of the flat punch compared to the sharp tip for nanoindentation results in a lower contact pressure. During the early stages of loading, the low pressure 
does not generate a high enough internal stress for dislocation nucleation, multiplication and slip to occur which prevents a sudden displacement of material or pop-in.

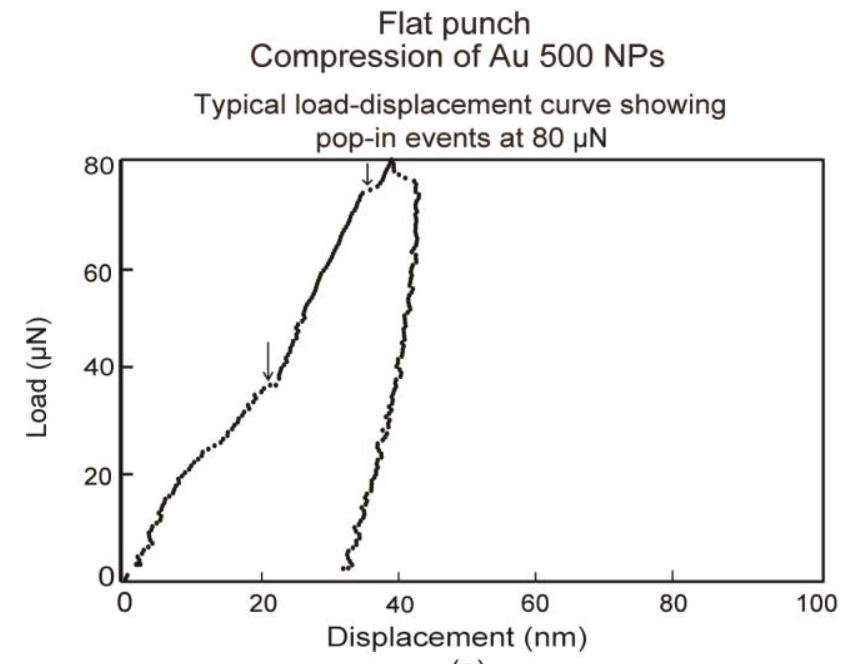

(a)
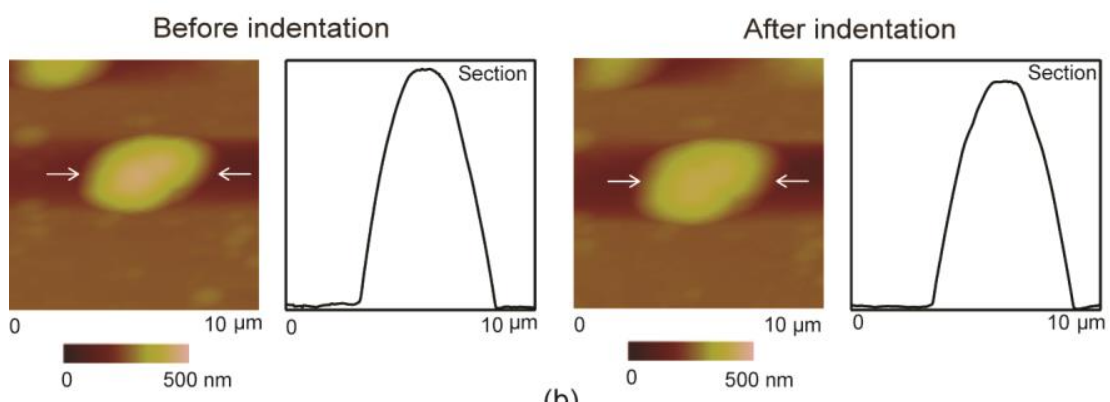

(Maharaj and Bhushan, 2014a)

Figure 32: (a) Typical load displacement compression curve at a maximum load of $80 \mu \mathrm{N}$ for Au 500 NPs with vertical arrows showing pop-in events and (b) topography maps and 2-D profiles at sections shown by the arrows before compression (first row) and after compression (second row) (Maharaj and Bhushan, 2014b). 
For Au 50 NPs and NRs typical load displacement curves for compression at a maximum load of $50 \mu \mathrm{N}$ s shown in Figure 33, along with topography maps of the nanoparticles over a $5 \mu \mathrm{m} \times 5 \mu \mathrm{m}$ scan area and 2-D profiles before and after compression. In Figure $33 \mathrm{~b}$ the horizontal white arrows indicate the nano-objects of interest along with the section on which the profiles were taken. In the after compression profiles, the solid line depicts time after $1 \mathrm{~min}$ and the dashed line time after $4 \mathrm{~min}$. The two profiles were examined to determine if there was recovery of the nano-objects as seen in previous studies (Yamakov et al., 2001; Gerberich et al., 2003). However, the before and after profiles, are essentially the same. In this case, due to the larger contact area, a lower pressure is being applied to the Au 50 NRs compared to the Au 50 NPs by the flat punch.

Figure 34 shows the topography maps of the nano-objects over a $5 \mu \mathrm{m} \times 5 \mu \mathrm{m}$ scan area and 2-D profiles before and after compression with a high load of $200 \mu \mathrm{N}$. In the after compression profiles, the solid line depicts time after $1 \mathrm{~min}$ and the dashed line time after 4 min. It can be observed that with Au 50 NPs and NRs there is some recovery as evidenced by the before and after profiles. Similar behavior was observed by Yamakov et al. (2001) using molecular dynamics (MD) simulation on nanocrystalline aluminum and Gerberich et al. (2003) during compression of Si nanoparticles using a nanoindenter. Under high stress, dislocations are generated and move towards the center of the grain. Upon removal of this stress, the dislocations glide back to their source, resulting in the 
Figure 33: Typical load-displacement curve at a maximum load of $50 \mu \mathrm{N}$ with $\mathrm{Au} 50 \mathrm{NPs}$ and NRs and (b) topography maps and 2-D profiles at sections shown by the horizontal arrows before compression (first row) and after compression (second row). The second row shows topography maps $1 \mathrm{~min}$ after compression and the solid lines and dashed lines show the 2-D profiles after 1 and 4 min respectively (Maharaj and Bhushan, 2014b). 
Flat punch

Compression of Au 50 NPs and NRs

Typical load-displacement curve showing pop-in events at $50 \mu \mathrm{N}$
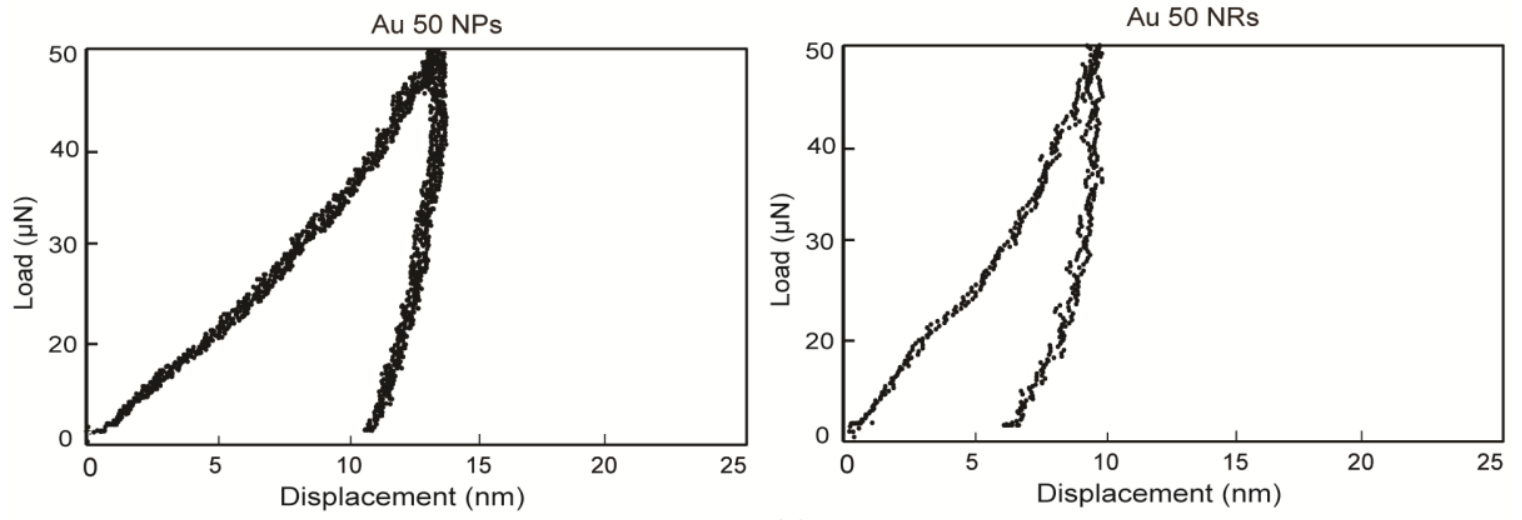

(a)

Example of compression of Au nanoparticles at a load of $50 \mu \mathrm{N}$

Before compression

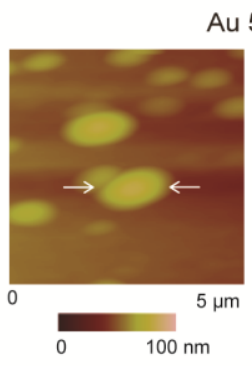

Au 50 NPs
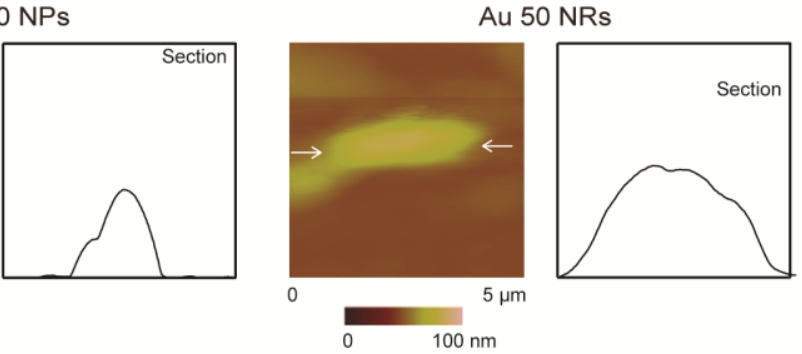

After compression
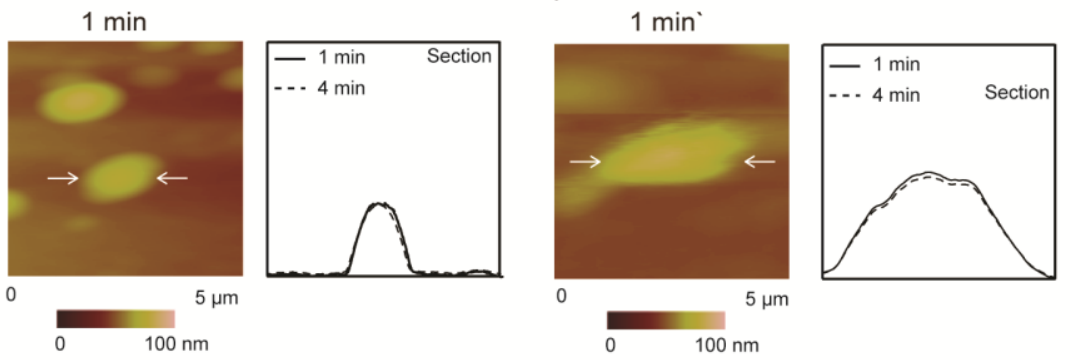

(b)

(Adapted from Maharaj and Bhushan, 2014b)

Figure 33 
Flat punch

Examples of compression of Au 50 NP and NR at $200 \mu \mathrm{N}$ and reverse plasticity after $4 \mathrm{~min}$

Before compression
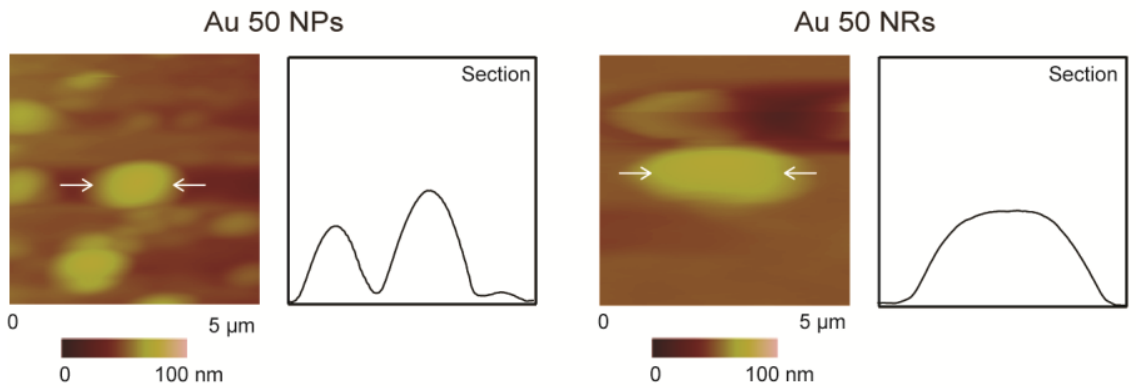

After compression
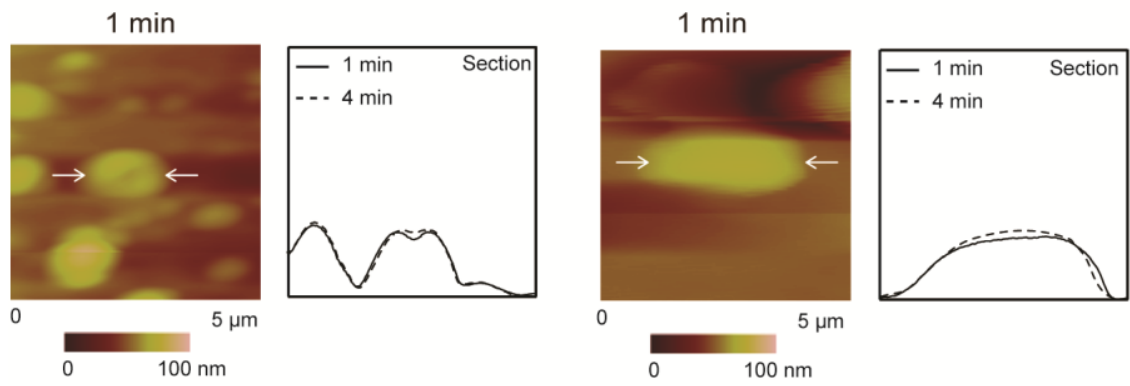

(Adapted from Maharaj and Bhushan, 2014b)

Figure 34: Topography maps and 2-D profiles at sections shown by the horizontal arrows before compression (first row) and after compression (second row) at a maximum load of $200 \mu \mathrm{N}$. The second row shows topography maps $1 \mathrm{~min}$ after compression and the solid lines and dashed lines show the 2-D profiles after 1 and 4 min respectively. Recovery of the nanoparticles 4 min after compression demonstrates reversed plasticity (Maharaj and Bhushan, 2014b). 
observed recovery or reverse plasticity. It is believed that this recovery can aid in the $\mathrm{Au}$ nano-objects retaining their spherical or cylindrical shapes, which helps to reduce friction and wear through rolling. In addition, this resistance to deformation prevents the nanoobjects from being easily flattened and this results in a reduced contact area which also leads to reduced friction and wear as previously discussed.

Repeat compression test were also performed with increasing loads for Au 500 NPs, Au 50 NPs 50 and Au 50 NRs. This provides an opportunity to study strain hardening on the nanoscale and investigate pop-in behavior as the loads are increased. Figure 35 shows load displacement curves for repeat loads for the nano-objects. Loads were applied in increasing increments of $50 \mu \mathrm{N}$ for a range $50-250 \mu \mathrm{N}$ for Au $500 \mathrm{NPs}$ and in increasing increments of $20 \mu \mathrm{N}$ for a range $50-110 \mu \mathrm{N}$ for Au 50 NPs 50 and NRs. For Au 500 NPs, initially as the load increases the displacement increases up to the $150 \mu \mathrm{N}$ load. For the 200 and $250 \mu \mathrm{N}$ loads, the displacements are almost the same as the $50 \mu \mathrm{N}$ load and less than the 100 and $250 \mu \mathrm{N}$ loads. For Au $50 \mathrm{NPs}$ and NRs as the loads are increased the displacements are either the same or less for each subsequent load displacement curve after $50 \mu \mathrm{N}$. This depicts a hardening effect up to the loads used. Higher loads were not possible as the nano-objects would either adhere to the indenter tip or slip out, resulting in the nano-object not being found during subsequent imaging. It is believed that the dislocations generated either continue to pile up or the already existing dislocations created during the previous loading phase prevents movement of new dislocations resulting in strain hardening. A larger number of pop-in events were observed especially at 200 and $250 \mu \mathrm{N}$ for Au 500 NPs. For NPs 50 and NRs 50 this 
Figure 35: Examples of repeat load-displacement curves for Au 50 NPs and NRs (Maharaj and Bhushan, 2014b) and Au 500 NPs (Maharaj and Bhushan, 2014a) with the corresponding maximum loads for each compression event. Evidence of strain hardening can be seen where successive loads results in the displacement being the same or less after unloading. 
Flat punch

Compression of Au nanoparticles and nanorods

Examples of repeat compression load-displacement curves showing strain hardening and pop-in events
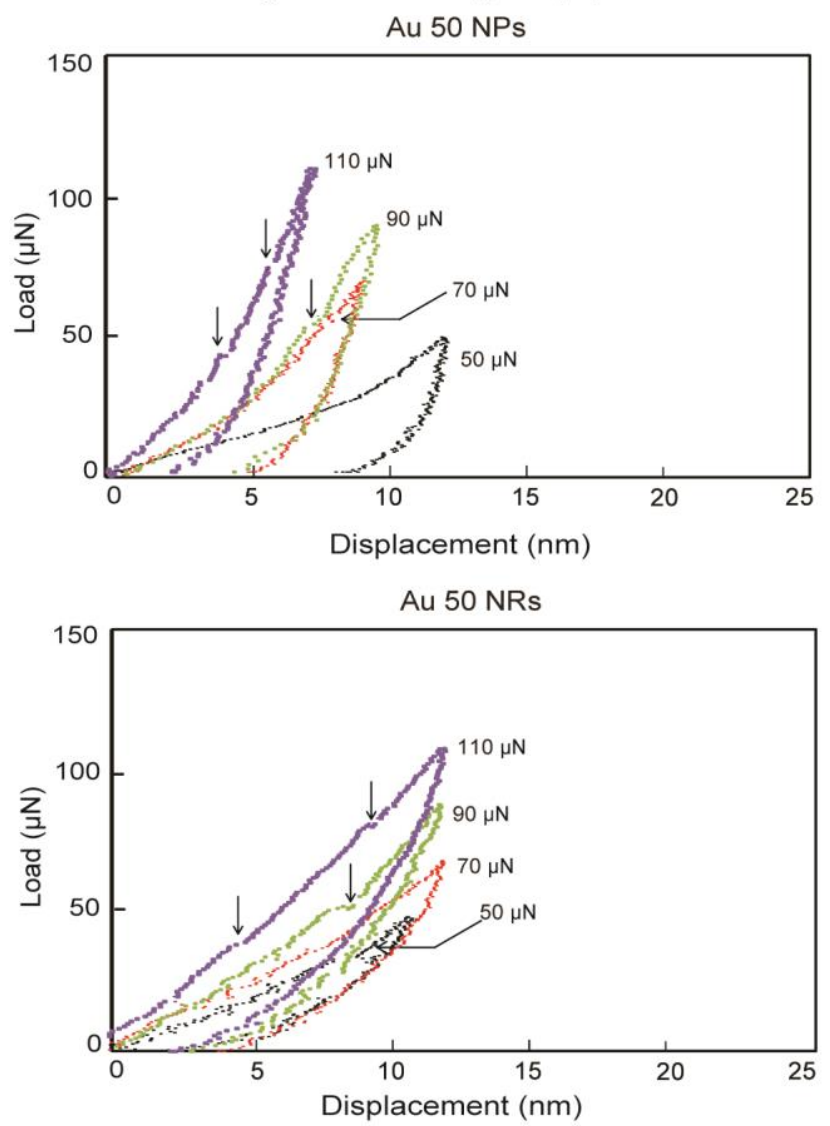

(Maharaj and Bhushan, 2014b)

(a)

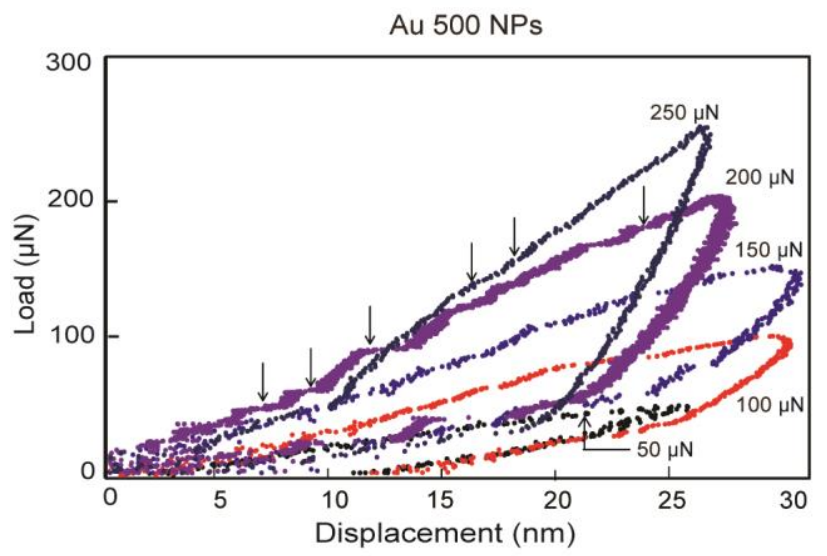

(Maharaj and Bhushan, 2014a)

(b)

Figure 35 
occurs at 90 and $110 \mu \mathrm{N}$. This indicates that the high stress generated by the accumulated dislocations from previous compressions, along with newly formed dislocations, results in multiple slip events occurring during loading. The increased resistance to deformation due to the hardening with the repeat compression can be useful in situations where repeated contacts occur in tribological systems on the macro- to nanoscale.

\subsection{Summary}

Au nanoparticles $50 \mathrm{~nm}$ and $500 \mathrm{~nm}$ in diameter, Au nanorods $50 \mathrm{~nm}$ in diameter and $200 \mathrm{~nm}$ in length along with a $100 \mathrm{~nm}$ thick film were investigated to determine their mechanical behavior on the nanoscale. Au 500 NPs and the Au film were investigated to determine their mechanical properties and to investigate scale effects through nanoindentation. Local deformation (nanoindentation) with a sharp tip and global deformation (compression) with a flat punch was performed on Au 500 in order to compare mechanical behavior during both types of deformation. Au 50 NPs and NRs were studied in compression to observe mechanical behavior during global deformation. This was performed under different loading regimes, with displacements from low to high. Hardening was investigated by compression using repeat loads.

For indentation with a sharp tip, an indentation size effect (ISE) was observed where the hardness of $\mathrm{Au} 500 \mathrm{NPs}$ and Au film increased due to higher strain gradients at shallower penetration depths. The hardness of the film was higher than the nanoparticles with both being higher than bulk due to Hall-Petch effect which was explained using the 
dislocation density mechanism and pile up mechanism with a greater contribution to the hardness coming from the dislocation density mechanism. TEM analysis confirmed the smaller grain sizes of the film compared to the nanoparticles. The large strain gradients also contributed to increased hardness compared to bulk Au. Load displacement curves for Au 500 NPs at low loads revealed pop-in effects, which occur due to generation and slip of dislocations. For the thin film, creep and strain rate tests showed displacements of a few nanometers for the hold period and increased loading-unloading times. This is believed to be due to diffusion creep associated with grain boundary translation.

For compression, pop-in effects from the sudden displacement of material as a result of accumulation and slip of dislocations, were observed during loading similar to nanoindentation for all nano-objects. For Au $50 \mathrm{NPs}$ and NRs reverse plasticity and deformation of the nano-objects were of interest. Displacement of the NRs was lower during loading compared to the NPs due to a larger contact area and the reduced contact pressure between the flat punch and NRs. This increased resistance to deformation is expected to allow NRs to be used under more severe loading conditions than NPs. Evidence of reverse plasticity was observed during unloading for the nano-objects in compression tests at high loads due to the high internal stresses generated within the nano-objects, which causes dislocations to retrace their path after unloading. It is believed that this recovery can aid in nano-objects retaining their spherical or cylindrical shapes which helps to reduce friction and wear through rolling. In addition, this resistance to deformation prevents the nano-objects from being easily flattened and results in a reduced contact area and lower friction and wear. 
Repeat compression tests showed a strain hardening effect with each subsequent load. The resulting displacement at each new load was either the same or lower than the previous. This was due to increased resistance to deformation as a result of a greater density of dislocations restricting the creation and movement of new dislocations being formed. Several pop-in effects were observed during repeat compression tests at increasing loads due to accumulation of dislocations from previous loads and formation of new dislocations. 


\section{Chapter 8: Mechanical behavior: Au NPs and NRs, CNHs and $\mathrm{MoS}_{2}$ and $\mathrm{WS}_{2}$ MWNTs}

\subsection{Introduction}

Results for nanoindentation using a Berkovich tip and compression using a flat punch are given for $\mathrm{MoS}_{2}$ MWNTs and only compression for $\mathrm{WS}_{2}$ MWNTs and CNHs. For both deformation methods, representative load-displacement curves are presented. Morphological characterization, before and after deformation, is also presented using a Berkovich tip. Finally load-displacement curves for repeat compression tests using the flat punch are presented.

\subsection{Nanoindentation}

\subsection{1. $\mathrm{MoS}_{2}$ MWNTs}

Indentation with a Berkovich tip was used to determine the mechanical properties of $\mathrm{MoS}_{2}$ MWNTs. This was done using displacement control at a maximum displacement of $50 \mathrm{~nm}$. Figure 36a shows a typical load-displacement curve with topography maps of the MWNTs over a $20 \mu \mathrm{m} \times 20 \mu \mathrm{m}$ scan area and 2-D profiles before and after indentation. The topography maps are used to confirm the indentation of the nanotubes and that the nanotube did not slip during loading and unloading. The vertical arrows on the load- 
Figure 36: (a) Typical load-displacement curve for low displacement of $50 \mathrm{~nm}$ for $\mathrm{MoS}_{2}$ MWNTs and topography maps and 2-D profiles, at sections indicated by the horizontal arrows before indentation (first row) and after indentation (second row). The dashed square highlights the indent in the "after indentation profile". (b) Typical load-displacement curve for high displacement of 100 nm. Vertical arrows point to pop-in events for both curves which occur due breaking of nanotube layers and sudden compression of separated layers. Indentation was performed on separate nanotubes for low and high displacement (Maharaj and Bhushan, 2015b). 
Berkovich tip

Indentation of $\mathrm{MoS}_{2}$ MWNTs

Typical load-displacement curve showing pop-in events at $50 \mathrm{~nm}$ as an example
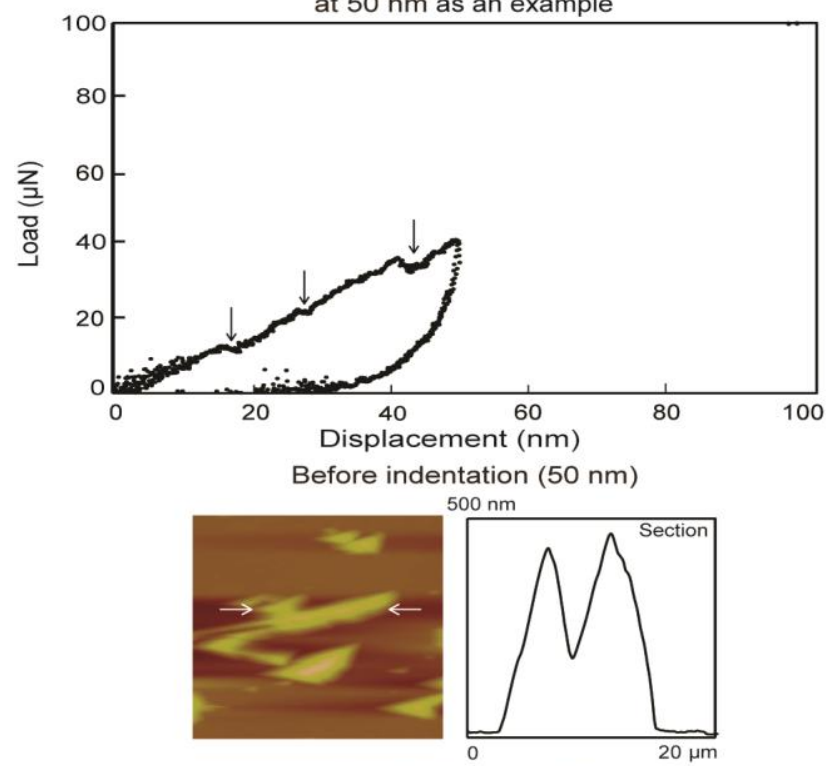

After indentation $(50 \mathrm{~nm})$

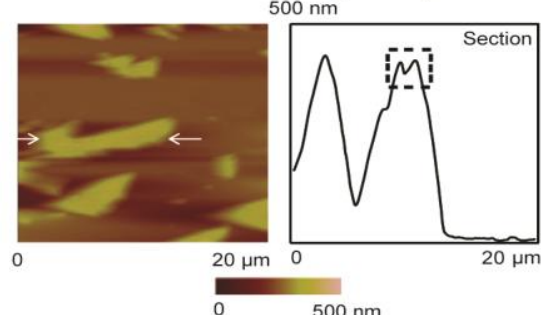

(a)

Typical load-displacement curve showing pop-in events

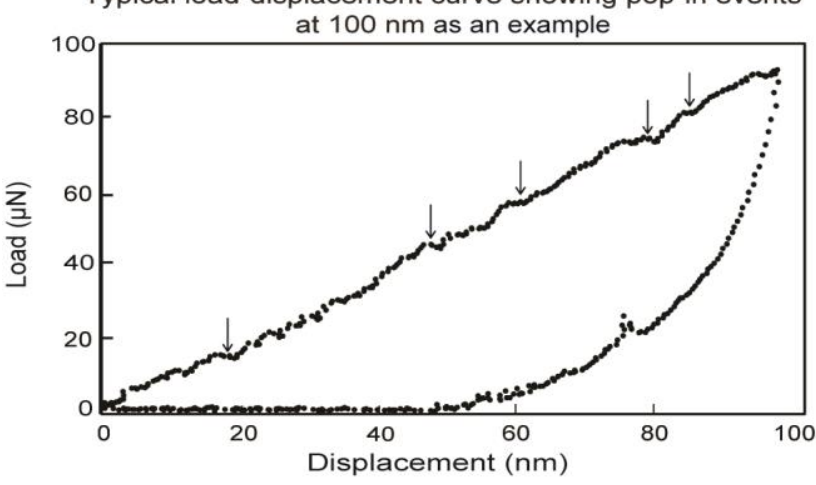

(b)

(Maharaj and Bhushan, 2015b)

Figure 36 
displacement curves point to pop-in events during indentation. The horizontal white arrows indicate the nanotube of interest along with the section on which the profiles were taken. The indent is highlighted by the dashed square on the "after indentation" profile and is not present on the "before indentation profile" at the same location.

Pop-ins have been observed in metallic and intermetallic materials by Goken and Kempf (2001) during initial steps of loading. They were reported to represent a sharp transition from pure elastic loading to a plastic deformation corresponding to an initial yield point. In the experiments with nanotubes pop-ins are believed to be as a result of displacement during breaking of the individual layers as well as sudden displacement through the spaces between the separated layers and already broken layers as shown in Figure 10a. The deformation that occurs during breaking of the layers is due to a combination of perfect and partial dislocations from stacking faults. Figure 7a-b shows stacking faults where Frank and Shockley partial dislocations form at the edge of the fault. Since the Frank partials are sessile, the breaking of the layers through plastic deformation occurs due to slip of perfect and Shockley partial dislocations (Maharaj and Bhushan, 2015b).

The hardness and Young's modulus were found at a displacement of $50 \mathrm{~nm}$ normal to the basal plane and are given in Table A.5 (see Appendix A). The hardness and Young's modulus for $\mathrm{MoS}_{2} \mathrm{MWNT}$ was found to be $0.6 \mathrm{GPa} \pm 0.2 \mathrm{GPa}$ and $12.5 \mathrm{GPa} \pm 4 \mathrm{GPa}$ respectively by Maharaj and Bhushan (2015b). Bulk MoS 2 has a similar hardness (0.6 GPa) and a Young's modulus of $52 \mathrm{GPa}$ normal to the basal plane. This was determined by Feldman (1976) based on neutron data by Wakabayashi et.al (1975) of a 2 mm thick $\mathrm{MoS}_{2}$ 
crystal. The lower Young's modulus of the $\mathrm{MoS}_{2} \mathrm{MWNT}$ s occurs as a result of the broken and separated layers present in the nanotube walls which provide less resistance to deformation (Figure 10a). The hardness of the $\mathrm{MoS}_{2}$ nanotube does not follow the smaller is stronger phenomenon seen in various nano-objects made of materials including $\mathrm{Au}, \mathrm{Cu}$, Nb and Ni (Thilly et al., 2002; Greer et al., 2005; Wu et al. , 2005; Shan et al., 2008; Dieteker et al., 2011, Mordehai et al., 2011a; Maharaj and Bhushan, 2014a).

In studies, for polycrystalline nano-objects the grain sizes were smaller than those found in bulk, which leads to dislocation pile-ups and an increased density of dislocations which entangle and resists plastic deformation (Li, 1969; Ashby, 1970; Gracio, 1994; Conrad and Jung, 2005). In the case of single crystalline nano-objects, dislocation starvation occurs where the absence of dislocations in the interior of the nano-object limits plastic deformation (Greer et al., 2005). Dislocation starvation is also not possible since stacking faults are observed in Figure 10a (Right) and both Frank and Shockley partial dislocations are present. In this case, separated layers, broken layers, and missing layers and Shockley dislocations decrease resistance to deformation and compete with Frank dislocations present which increase resistance to deformation, as explained in a previous section in 2.3. These competing effects result in no appreciable increase in hardness (Maharaj and Bhushan, 2015b).

To further understand deformation during indentation, higher displacements were also explored. Figure $36 \mathrm{~b}$ shows indentation at a displacement close to $100 \mathrm{~nm}$ with a larger number of pop-ins compared to displacement of $50 \mathrm{~nm}$. This is due to a greater number of defects, such as broken and separated layers, encountered by the diamond tip as 
the layers are penetrated. The broken and separated layers can lead to exfoliation as they are easier to peel apart at higher loads.

In tribological applications this can be beneficial as exfoliation leads to formation of an easily sheared tribofilm which results in reduction in friction and wear (Maharaj et al., 2013).

In summary, the load-displacement curves for $\mathrm{MoS}_{2} \mathrm{MWNTs}$ revealed several pop-in effects which result from slip as the sharp tip penetrates broken layers and spaces between separated layers. No scale effects were observed. The thin walls of the MWNTs does not allow for generation of a sufficient number of dislocations to support the density and pile up mechanisms necessary for increased hardness. Sessile Frank partials are also limited by the thin walls and do not contribute significantly to hardening to offset the deformation caused by perfect and Shockley partial dislocations. Separation of layers and broken layers also results in less resistance to displacement of the sharp tip during indentation.

\subsubsection{Compression: $\mathrm{MoS}_{2}$ and $\mathrm{WS}_{2}$ MWNTs and CNHs}

Nano-objects were compressed to understand global deformation (compression) behavior and compare to local deformation (indentation). For this purpose, a tip approximately $3.5 \mu \mathrm{m}$ in radius was used to carry out compression tests. Compression data for the MWNTs are presented first followed by CNHs.

Figure 37 shows typical load displacement curves for compression at maximum displacements of 50, 100 and $300 \mathrm{~nm}$ for $\mathrm{MoS}_{2}$ and $\mathrm{WS}_{2} \mathrm{MWNTs}$. The vertical arrows 
Figure 37: Compression load displacement curves for displacements of $50 \mathrm{~nm}, 100 \mathrm{~nm}$ and $300 \mathrm{~nm}$ for $\mathrm{MoS}_{2}$ (Left) and WS 2 MWNTs (Right) with the vertical arrows depicting pop-in events. Magnified images are shown inset for displacements of $50 \mathrm{~nm}$ to more clearly illustrate pop-in events which are a result of breaking of individual layers of the nanotubes and sudden compression of separated layers (Maharaj and Bhushan, 2015b). 
Flat punch

Compression of $\mathrm{MoS}_{2}$ MWNTs Compression of $\mathrm{WS}_{2}$ MWNTs

Typical load-displacement curve showing pop-in events at $50 \mathrm{~nm}$ as an example
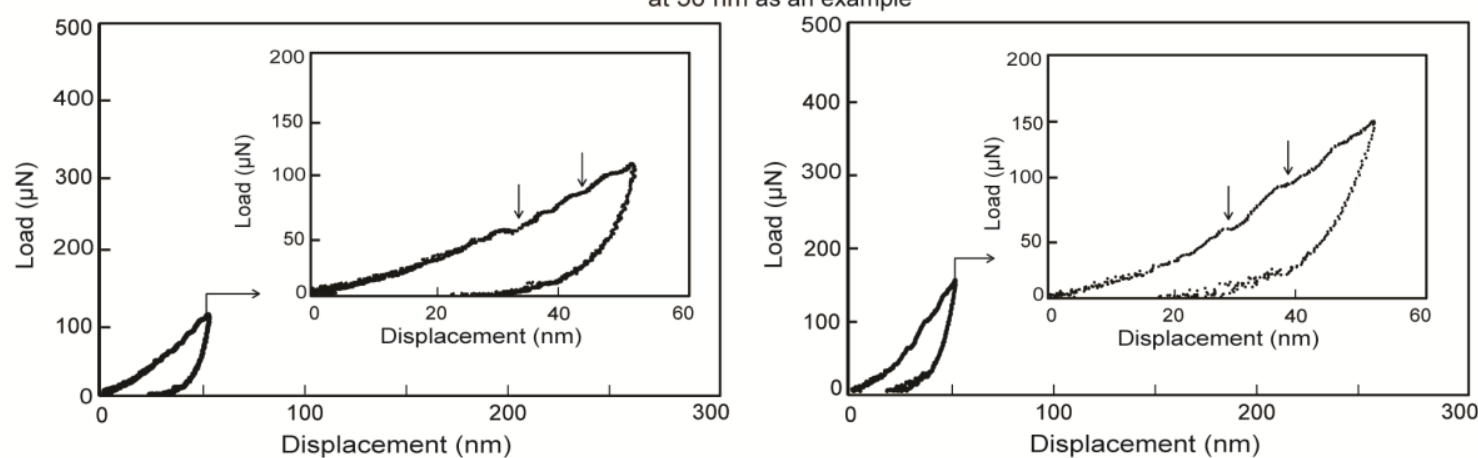

Typical load-displacement curve showing pop-in events at $100 \mathrm{~nm}$ as an example
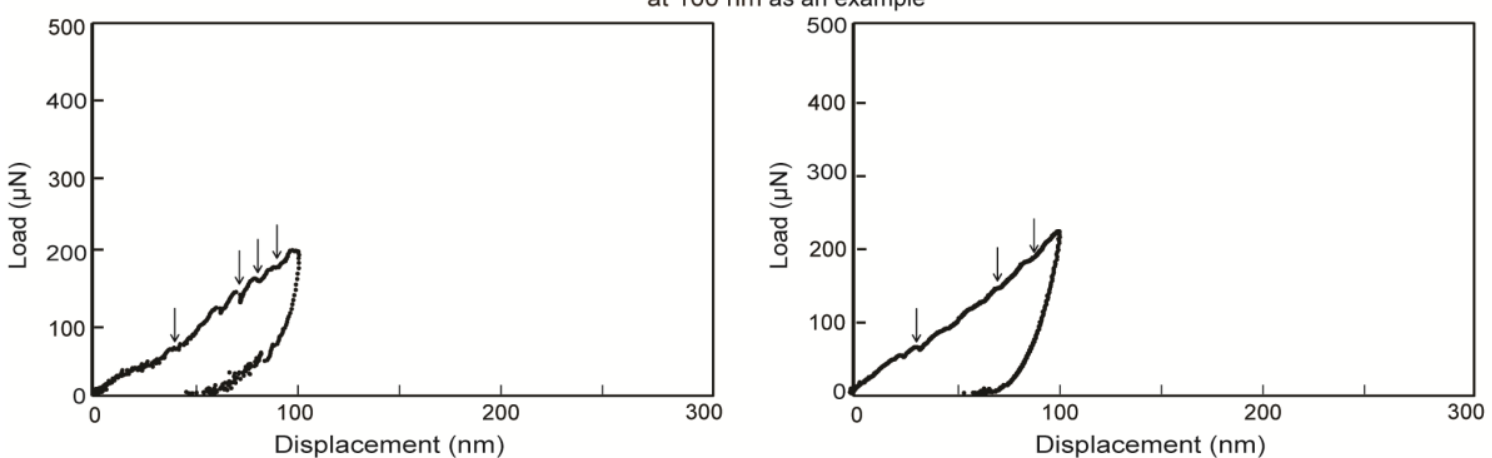

Typical load-displacement curve showing pop-in events at $300 \mathrm{~nm}$ as an example
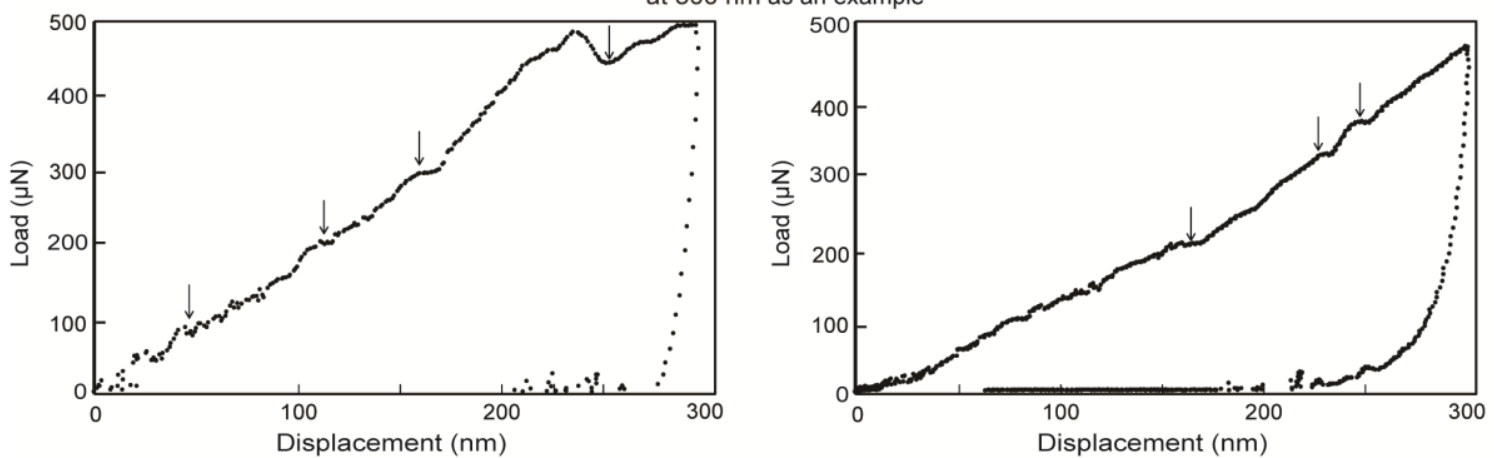

(Maharaj and Bhushan, 2015b)

Figure 37 
point to some examples of pop-in events during compression of the nanotubes. These events similar to indentation are a result of breaking of the layers of the walls of the $\mathrm{MoS}_{2}$ and $\mathrm{WS}_{2}$ nanotubes where plastic deformation occurs as a result of slip of perfect and Shockley partial dislocations. In the case of $\mathrm{MoS}_{2} \mathrm{MWNTs}$, compression of spaces between separated layers also contributes to pop-ins. For the $\mathrm{MoS}_{2} \mathrm{MWNT}$ s there are less pop-in events during compression compared to indentation (Figure 36). Here, the entire nanotube is compressed and there is more resistance to deformation and breaking of the layers of the nanotube walls. For WS 2 MWNTs, a greater load is required for the same displacement $(50 \mathrm{~nm})$ as compared to $\mathrm{MoS}_{2}$ MWNTs. At a depth as low as $50 \mathrm{~nm}$, hardening due to substrate effects are unlikely as the displacement is less than $30 \%$ (annonymous, 1979) of the height of the nanotubes. The higher resistance to deformation of the $\mathrm{WS}_{2}$ MWNTs, is due to fewer defects (Figure 10b), as there are no separated layers or broken layers present as in the case of $\mathrm{MoS}_{2} \mathrm{MWNT}$. However, even though there are more pop-ins at higher displacements (100 nm and $300 \mathrm{~nm}$ ) for $\mathrm{MoS}_{2} \mathrm{MWNTs}$, the difference in load between the $\mathrm{MoS}_{2}$ and $\mathrm{WS}_{2}$ MWNTS is less as the substrate begins to influence the resistance to deformation and creates a hardening effect (Maharaj and Bhushan, 2015b).

The broken layers of $\mathrm{MoS}_{2}$ MWNTS shown in Figure 10a (Center) and can lead to exfoliation as the layers come apart under increasing load. This, along with already exfoliated layers (center) on $\mathrm{WS}_{2}$ MWNTs as shown in Figure 10b (Center), can lead to formation of an easily shearable film during sliding in tribological applications, which reduces friction. For $\mathrm{WS}_{2} \mathrm{MWNTs}$, greater resistance to compression can also be 
beneficial for reduction of friction and wear. In instances where there is high moisture content, $\mathrm{MoO}_{3}$ and $\mathrm{WO}_{3}$ can be formed as $\mathrm{MoS}_{2}$ and $\mathrm{WS}_{2}$ become oxidized in the presence of oxygen. In these situations the tribofilm formation is interrupted as layers clump together. The water vapor present can also condense in the defects of the crystal structure as shown in Figure 10a, b and would interrupt the easy shear of the basal planes (Pope and Pannitz, 1988; Uemura et al., 1990; Prasad and Zabinski, 1993). Since the $\mathrm{WS}_{2}$ MWNTs offer better resistance to deformation compared to the $\mathrm{MoS}_{2} \mathrm{MWNTs}$, these may be more suitable for friction and wear reduction in humid conditions. In this case since the nanotube layers are not as easily exfoliated and the friction and wear reduction is as a result of reduced contact area, sliding and possible rolling (Maharaj et al., 2013).

Compression data for CNHs are now presented and compared to the MWNTs. Figure 38 shows load-displacement compression curves for $\mathrm{CNHs}$ for displacements of 25, 50 and $75 \mathrm{~nm}$ with vertical arrows pointing to pop-in events. Lower displacements were chosen for the compression due to the smaller diameter of the nanoparticles ( $80-$ $100 \mathrm{~nm}$ ). Similar to the $\mathrm{MoS}_{2}$ and $\mathrm{WS}_{2}$ MWNTs, pop-in events occur with CNHs at all three displacements. The single layer graphene horns which comprise CNHs, have a high hardness of $950 \mathrm{GPa}$ and Young's modulus of $1000 \mathrm{GPa}$ as shown in Table A.5 (see Appendix A), but at the same time is very flexible and can easily bend (Scarpa et.al, 2009). The pop-ins for CNHs occurs as a result of buckling of the individual nanohorns. For a displacement of $50 \mathrm{~nm}$ a lower load $(80 \mu \mathrm{N})$ for the $\mathrm{CNHs}$ is required to produce 
the same displacement compared the $\mathrm{MoS}_{2}$ and $\mathrm{WS}_{2}$ MWNTs, shown in Figure 37 (108 $\mu \mathrm{N}$ and $150 \mu \mathrm{N})$, due to the flexibility of the CNHs.

For tribological applications under high loads, rolling and sliding of spherical CNHs may not be possible due to their flexible nature and ability to deform easily. The individual nanohorns can still reduce friction and wear since they will not break apart easily due to the high hardness and Young's modulus and would provide an easily shearable layer for sliding which would reduce friction and wear. CNHs also have a lower work of adhesion compared to $\mathrm{MoS}_{2}$ and $\mathrm{WS}_{2}$ which leads to lower meniscus contribution to adhesion (Maharaj et al., 2013). In this way the graphene layers provides a low shear strength surface for sliding in both dry and humid conditions.

In summary, $\mathrm{MoS}_{2}$ and $\mathrm{WS}_{2} \mathrm{MWNTs}$ and $\mathrm{CNHs}$, pop-ins are observed under low to high displacements. The mechanism for the MWNTs is different from the CNHs, where the individual walls breaks compared to buckling of the individual nanohorns which are flexible.

This flexibility was further seen where, for a comparable displacement for all three nano-objects, CNHs required the lowest load. Further compression tests to study hardening effects of the nano-objects were also performed.

Repeat compression tests were performed at a constant displacement of $50 \mathrm{~nm}$ for the $\mathrm{MoS}_{2}$ and $\mathrm{WS}_{2} \mathrm{MWNTs}$ and $25 \mathrm{~nm}$ for the CNHs. This provides an opportunity to study hardening effects on the nanoscale. Figure 39 shows load displacement curves for repeat compression for the nano-objects. For all cases higher consecutive loads are required to produce the same displacements. This depicts a hardening effect up to the 
Flat punch

Compression of $\mathrm{CNH}$ with Displacement of 25, 50 and $75 \mathrm{~nm}$

Typical load-displacement curve showing pop-in events

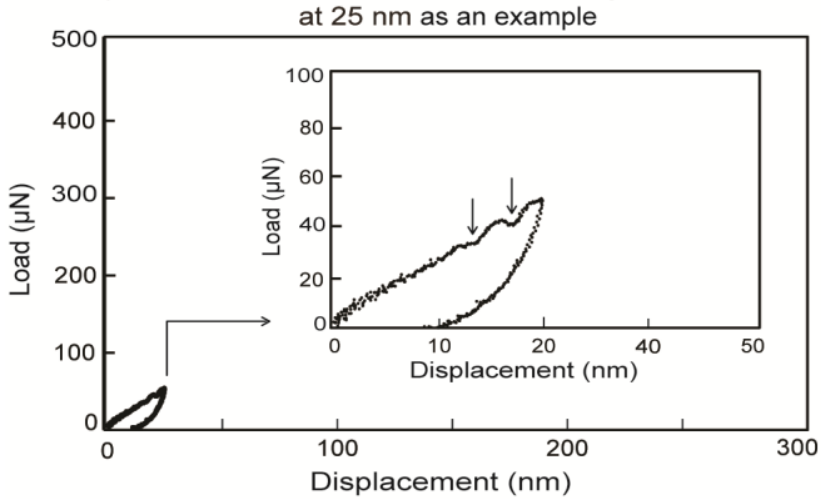

Typical load-displacement curve showing pop-in events at $50 \mathrm{~nm}$ as an example



Typical load-displacement curve showing pop-in events



Figure 38: Compression load displacement curves for displacements of $25 \mathrm{~nm}, 50 \mathrm{~nm}$ and $75 \mathrm{~nm}$ for CNHs with the vertical arrows showing examples of pop-in events. Here pop-in events are a result from buckling of individual nanohorns (Maharaj and Bhushan, 2015b). 
loads used. The layers of the $\mathrm{MoS}_{2}$ and $\mathrm{WS}_{2}$ nanotube walls are pressed together and the spaces between them become smaller. As they are pressed into the substrate a higher load is required to produce the same displacement for each successive run. The vertical arrows point to examples of pop-ins as individual layers that comprise the nanotube walls break. For the CNHs, as individuals nanohorns buckle and are pressed onto each other and the substrate, further compression becomes more difficult, similar to the MWNTs. Pop-ins in this case occur due to buckling of the individual nanohorns.

In summary, all three nano-objects display pop-in effects during loading and increased resistance to deformation during repeat compression tests. $\mathrm{CNHs}$ required the smallest load for a displacement of $50 \mathrm{~nm}$ compared to the MWNTs. This is due to buckling of the individual nanohorns as a result of their flexible nature.

The increased resistance to deformation with the repeat compression can be useful in situations where repeated contacts occur in tribological systems on the macro- to nanoscale as deformation of the nano-objects becomes more difficult. 
Figure 39: Examples of repeat load-displacement curves for $\mathrm{MoS}_{2}$ and $\mathrm{WS}_{2} \mathrm{MWNTs}$ at a constant displacement of $50 \mathrm{~nm}$ and CNHs at a constant displacement of 25 nm. Vertical arrows point to pop-in events. Pop-in events for the nanotubes occur due to breaking of walls, and sudden compression of separated layers. For the $\mathrm{CNH}$ nanoparticle pop-in events occur due to buckling of individual nanohorns. Evidence hardening can be seen for all three cases where the loads increase at a constant displacement due to compression of individual layers and of MWNTs and nanohorns (Maharaj and Bhushan, 2015b). 
Flat punch

Compression of nano-objects

Examples of repeat compression load-displacement curves showing hardening and pop-in events

$\mathrm{MoS}_{2}$
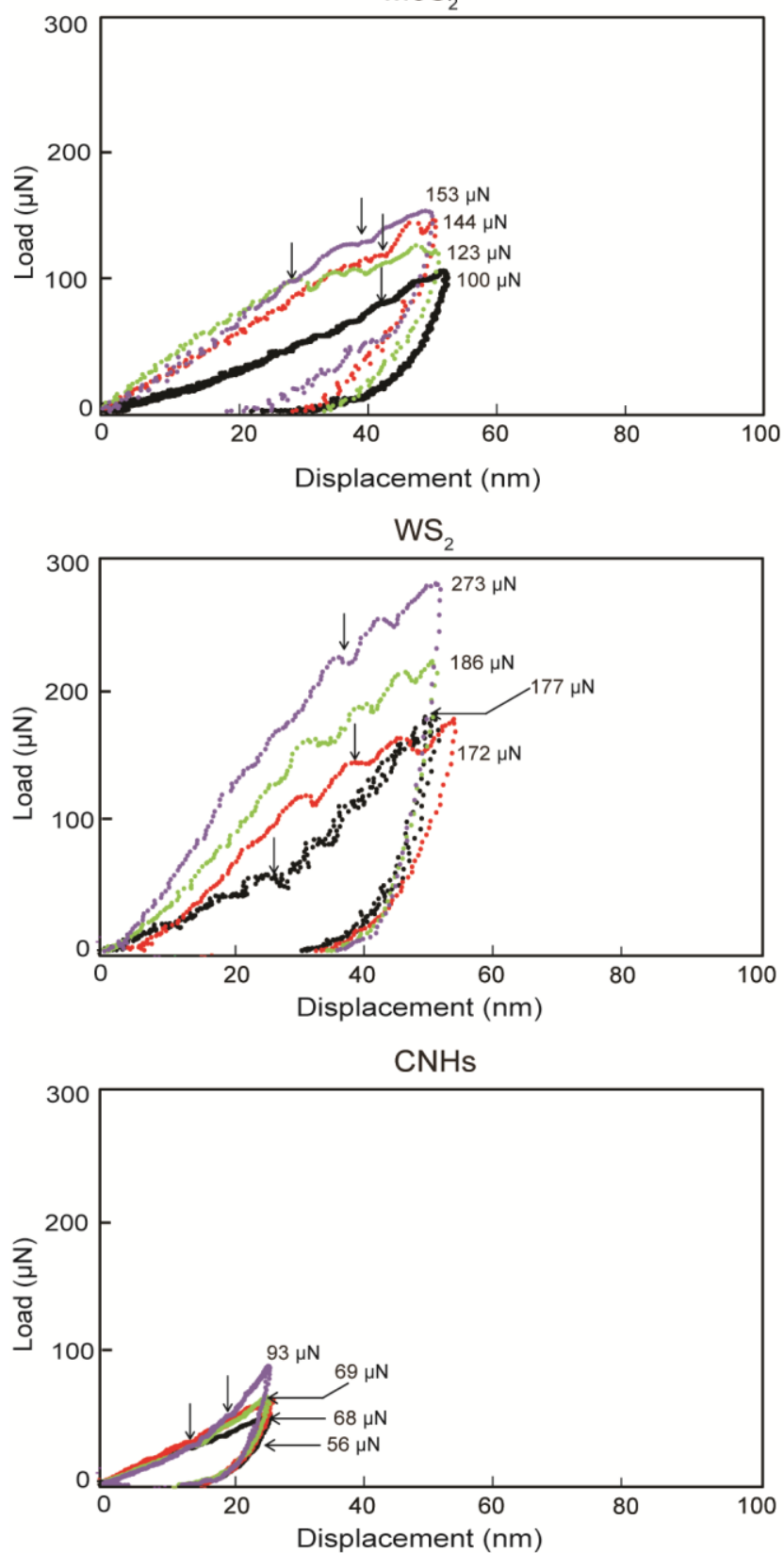

(Maharaj and Bhushan, 2015b)

Figure 39 


\subsection{Summary}

$\mathrm{MoS}_{2}\left(500 \mathrm{~nm}\right.$ in diameter) and $\mathrm{WS}_{2}$ MWNTs (300 $\mathrm{nm}$ in diameter) and CNHs (80-100 $\mathrm{nm}$ in diameter) were investigated to determine their mechanical behavior. $\mathrm{MoS}_{2}$ MWNTs were investigated to study scale effects and local deformation (nanoindentation) with a sharp tip. Nanoindentation was performed only on the $\mathrm{MoS}_{2} \mathrm{MWNTs}$. Since decreasing nano-object size results in less contact with the tip due to the curvature of both the tip and nano-object, this can lead to inaccuracies in determining contact area and consequently mechanical properties. The large diameter of the $\mathrm{MoS}_{2} \mathrm{MWNT}$ provides a flatter surface for nanoindentation compared to the other nano-objects and leads to less error in determination of mechanical properties. Global deformation (compression) studies were also performed on the $\mathrm{MoS}_{2} \mathrm{MWNT}$ s with a flat punch using a depth sensing nanoindenter in order to compare mechanical behavior during both types of deformation. $\mathrm{WS}_{2} \mathrm{MWNT}$ and $\mathrm{CNHs}$ were studied in compression only to observe mechanical behavior during global deformation. This was performed under different displacement regimes, with displacements from low to high. Hardening was investigated by compression using repeat loads.

For indentation with a sharp tip, the load-displacement curves revealed several pop-in effects which result from slip as the sharp tip penetrates broken layers and spaces between separated layers. The hardness of the $\mathrm{MoS}_{2}$ MWNTs was found to be similar to bulk and does not follow a smaller is stronger trend. The thin walls of the nanotube does not allow for generation of a sufficient number of dislocations to support the density and pile up mechanisms necessary for increased hardness. Sessile Frank partials are also 
limited by the thin walls and do not contribute significantly to hardening to offset the deformation caused by perfect and Shockley partial dislocations. Separation of layers and broken layers also results in less resistance to displacement of the sharp tip during indentation.

For compression, pop-in effects from the sudden displacement of material were observed in both MWNTs during loading, similar to nanoindentation for MWNTs. For CNHs pop-ins were also observed and resulted from buckling of the horns of the nanoparticle. The defected layers of $\mathrm{MoS}_{2}$ MWNTs and the flexibility of the graphene results in less resistance to deformation compared to $\mathrm{WS}_{2} \mathrm{MWNTs}$, which do not show separated and broken layers observed in $\mathrm{MoS}_{2}$ MWNTs. Repeat compression tests showed a hardening effect with each subsequent load at constant displacement. The resulting load for constant displacement was either the same or higher than the previous. For the nanotubes, this was due to increased resistance to deformation as a result of the layers of the nanotubes being pressed together. For the $\mathrm{CNHs}$, individual horns buckle and are pressed against each other and onto the substrate making further compression more difficult.

All three nano-objects display pop-in effect during loading and increased resistance to deformation during repeat compression tests. $\mathrm{CNHs}$ required the smallest load for a displacement of $50 \mathrm{~nm}$ compared to the MWNTs. This is due to buckling of the individual nanohorns as a result of their flexible nature. 


\section{Chapter 9: Conclusions and Outlook}

Nano-objects have been used in numerous applications on the macro- to nanoscale where they come into contact with each other or surfaces on which they are used. Nano-objects of much interest are Au nanoparticles and nanorods, $\mathrm{MoS}_{2}$ and $\mathrm{WS}_{2}$ MWNTs and CNHs due to their widespread use in many applications. In this thesis studies on these nano-objects are carried out to gain an understanding of interfacial friction and wear, their mechanical properties and deformation mechanisms. A summary of the studies along with the observations from each study are given in Figure 3.

Single nano-object contact studies in dry and submerged in liquid environments help to understand friction mechanisms of a nano-object sliding on surfaces. Multiple nano-object contact studies demonstrate the ability of several nano-objects to reduce friction and wear in dry and submerged in liquid environments. Further studies on friction mechanisms have been done by sliding on single nano-objects such as $\mathrm{MoS}_{2}$ and $\mathrm{WS}_{2}$ MWNTs. These studies provide an understanding of the effects of topography, orientation, and scale on friction. Nano-objects have also been investigated during local deformation (indentation) and global deformation (compression) to study of mechanical behavior on the nanoscale. Local deformation studies allow for investigation of mechanical properties and scale effects. Comparisons between loading methods help understand deformation mechanisms.

Single nano-object contact studies revealed a friction force dependence on contact area for both dry and submerged in liquid environments. Studies also suggest that increased probability of rolling lowers friction force when comparing spherical nanoobjects to cylindrical. Material and roughness effects were also observed where nano- 
objects with higher roughness and lower work of adhesion show the lowest friction forces, as in the case of CNHs. Elimination of meniscus forces in submerged in liquid environments and presence of a low shear strength surface further reduces friction forces.

In multiple nano-object contact studies, reduced real area of contact and the ability of nano-objects to roll and slide reduces coefficients of friction compared to sliding on a surface without nano-objects. Similar mechanisms, as in single nano-object contact, contribute to lower friction. Wear studies reveal that addition of nano-objects results in lower wear by reducing the real area of contact between sliding surfaces and facilitating ease of shearing.

Macroscale friction and wear studies show similar trends to the nanoscale. Friction and wear reduction also occurs due to exfoliation and tribofilm formation of lamellar nano-objects, such as $\mathrm{MoS}_{2}$ and $\mathrm{WS}_{2}$ MWNTs in dry environments.

To further understand friction mechanisms, single nano-objects such as $\mathrm{MoS}_{2}$ and WS 2 MWNTs have been studied for effects of topography, orientation, and scale effects. Studies show variations in friction coincide with changes in topography and are attributed to ratchet mechanism and "collision" effects. Scale effects are observed with lower friction on the nanoscale compared to macroscale due to reduced contact area for several materials. Studies also show environmental effects on friction. Here, increased humidity can lead to oxidization of surfaces and together with condensation of water between layers, can prevent ultra-low friction.

In nanoindentation studies, for a $200 \mathrm{~nm}$ thick Au film higher hardness was observed when compared to Au nanoparticles $500 \mathrm{~nm}$ in diameter due to the smaller 
grain size of the film. Both materials showed higher hardness compared to bulk due to smaller grain size. This is explained in terms of the dislocation pile-up and density mechanism. Materials also show higher hardness at shallower depths due to higher strain gradients. Load displacement curves show increased pop-ins, which occur due to generation and slip of dislocations. Other studies of local deformation, showed pop-in behavior from slip as the sharp tip penetrates defects such as broken layers and spaces between separated layers of $\mathrm{MoS}_{2}$ MWNTs. Sessile partial dislocations generated are not sufficient to offset the deformation caused by perfect and partial glissile dislocations. Together with defects this provides less resistance to deformation and hardness was similar to bulk.

Compression of several nano-objects has been performed. For Au nano-objects, pop-in effects were observed during loading, similar to nanoindentation. For MWNTs, pop-ins occur due to penetration of defects, as in nanoindentation. For CNHs, they were the result of buckling of the individual horns of the nanoparticle. The defected layers of $\mathrm{MoS}_{2}$ MWNTs and the flexibility of the graphene results in less resistance to deformation compared to $\mathrm{WS}_{2}$ MWNTs which do not show separated and broken layers observed in $\mathrm{MoS}_{2}$ MWNTs. In Au NPs and NRs, $50 \mathrm{~nm}$ in diameter, reversed plasticity was observed due to generation of high internal stresses which causes dislocations to retrace their path upon unloading.

Studies of nano-objects in repeat compression show increased resistance for all nano-objects. In Au nano-objects, this occurs as a result of accumulation of dislocations, which prevents formation of new dislocations and slip of already existing dislocations. 
For MWNTs, this is as a result of individual layers of the nanotubes being pressed together. For CNHs, individual nanohorns buckle and are pressed against each other and the substrate making further compression more difficult.

In summary, studies on Au nanoparticles and nanorods, $\mathrm{MoS}_{2}$ and $\mathrm{WS}_{2} \mathrm{MWNTs}$ and CNHs were performed to understand friction, wear and mechanical behavior. Studies on single and multiple nano-object contact in dry and submerged in liquid environments, on friction on single nano-objects and on mechanical behavior during indentation and compression were examined.

In single nano-object contact, friction at the initiation of sliding on surfaces was influenced by real area of contact, roughness and work of adhesion. In submerged in liquid environments, elimination of meniscus forces and the presence of a low shear strength film, reduces friction compared to dry environments. In multiple nano-object contact friction and wear reduction occurs due to the presence of nano-objects which lowers the real area of contact between sliding surfaces. Exfoliation and tribofilm formation contributes to friction and wear reduction on the macroscale in layered materials. Further friction studies performed by sliding on single nano-objects showed the influence of topography on friction due to the ratchet mechanism and 'collision' effects. Lower friction was observed on the nanoscale compared to the macroscale. The presence of moisture results in changes in material composition leading to higher friction.

Deformation studies were performed, which aids in understanding how nanoobjects behave during loading and unloading in friction and wear reduction. Studies examined scale effects of mechanical properties and compared mechanical behavior 
between local (indentation) and global (compression) deformation. For some nanoobjects, studies show mechanical properties can be enhanced which is dependent on grain size and is dislocation driven. In other cases where mechanical properties showed no improvement, it was found that structural defects were responsible for lower resistance to deformation. Reverse plasticity was also observed due to high internal stresses during compression. Increased resistance to repeat compression is due to accumulation of dislocations or compression of nano-object layers.

Future nano-object studies may incorporate magnetic alignment of metallic nanotubes at different orientations in multiple nano-object contact. This would help to determine the effect of orientation on rolling and friction reduction. In deformation 
studies more work has to be done with nano-objects of different materials with varying grain sizes to predict the onset of reverse plasticity. Computer simulations will prove useful in quantifying the number of dislocations needed for such effects. More studies need to be done on the role of external physical dimensions of nano-objects on mechanical properties for both single and polycrystalline materials for increasingly smaller dimensions.

The knowledge gained from these studies in this thesis will further aid in understanding fundamental interfacial interactions and deformation mechanisms of nanoobjects. 
Appendix A: Tables 
Table A.1: Review of studies of enhanced scale-dependent mechanical properties of bulk solid surfaces, thin films and various nano-objects. For thin films, the thicknesses are given and for nano-objects, the diameters are given. Pillars described in the studies have low aspect ratios (2-20) compared to wires (> 20) (Adapted from Maharaj and Bhushan 2014a).

${ }^{\mathrm{a}}$ Gane and Cox (1970), ${ }^{\mathrm{b}}$ Pethica et al. (1983), ${ }^{\mathrm{c}}$ Pharr and Oliver (1988), ${ }^{\mathrm{d}}$ Bhushan and Koinkar (1994), ${ }^{\text {e } B h u s h a n ~ e t ~ a l . ~(1996), ~}{ }^{\mathrm{f}}$ Mante et al., (1999), ${ }^{\mathrm{g}}$ Grillo et al.(2003), h Doerner et al. (1986), ${ }^{\mathrm{i}}$ Cao et al. (2006), ${ }^{\mathrm{j}}$ Dietiker et al. (2008), ${ }^{\mathrm{k}}$ Maharaj and Bhushan (2014a), ${ }^{1}$ Knapp and Follstaedt. (2004), ${ }^{\mathrm{m}}$ Thilly et al. (2002), ${ }^{\mathrm{n}}$ Greer et al. (2005), ${ }^{\mathrm{o}} \mathrm{Wu}$ et al. (2005), ${ }^{\mathrm{p}}$ Shan et al. (2008), ${ }^{\mathrm{q}}$ Dieteker et al. (2011), ${ }^{\mathrm{r}}$ Mordehai et al.,(2011b), ${ }^{\mathrm{s}}$ Fleck and Hutchinson (1993), ${ }^{\mathrm{t}} \mathrm{Nix}$ and Gao (1998), ${ }^{\mathrm{u}} \mathrm{Hall}$ (1951), ${ }^{\mathrm{v}}$ Petch (1953), ${ }^{\mathrm{w}} \mathrm{Li}$, (1969), ${ }^{\mathrm{x}}$ Abson and Jonas (1970), ${ }^{\mathrm{y}}$ Nix (1989). 


\begin{tabular}{|c|c|c|c|}
\hline Material & Method & Result & Theories \\
\hline \multicolumn{4}{|c|}{ Solid surfaces } \\
\hline $\begin{array}{l}\mathrm{Ag}, \mathrm{Au}, \mathrm{Cu}, \mathrm{GaAs}, \mathrm{GaP}, \mathrm{Ni} \\
\mathrm{Si}, \mathrm{Ti}, \mathrm{ZnSe}\end{array}$ & Indentation $^{\mathrm{a}-\mathrm{g}}$ & $\begin{array}{l}\text { Hardness inversely proportional to indentation } \\
\text { depth. }\end{array}$ & Strain gradient plasticity ${ }^{\mathrm{s}, \mathrm{t}}$ \\
\hline \multicolumn{4}{|c|}{ Thin films } \\
\hline $\mathrm{Al}(210-1090 \mathrm{~nm})$ & Indentation $^{\mathrm{h}}$ & \multirow[t]{3}{*}{$\begin{array}{l}\text { Hardness inversely proportional to film } \\
\text { thickness. }\end{array}$} & $\begin{array}{l}\text { Hall-Petch }{ }^{\mathrm{u}-\mathrm{x}} \\
\text { Dislocation constraint }{ }^{\mathrm{x}}\end{array}$ \\
\hline $\mathrm{Ag}, \mathrm{Au}(100-2000 \mathrm{~nm})$ & Indentation $^{\mathrm{i}}$ & & \\
\hline $\mathrm{Au}(31-858 \mathrm{~nm})$ & Indentation $^{\mathrm{j}}$ & & Hall-Petch ${ }^{\mathrm{u}-\mathrm{x}}$ \\
\hline $\mathrm{Au}(100 \mathrm{~nm})$ & Indentation $^{\mathrm{k}}$ & $\begin{array}{l}\text { Hardness inversely proportional to indentation } \\
\text { depth and higher than bulk }\end{array}$ & $\begin{array}{l}\text { Hall-Petch }^{\mathrm{u}-\mathrm{x}} \\
\text { Strain gradient plasticity } \\
\end{array}$ \\
\hline $\mathrm{Ni}(50-700 \mathrm{~nm})$ & Indentation $^{1}$ & Hardness higher than bulk & Dislocation constraint ${ }^{\mathrm{x}}$ \\
\hline \multicolumn{4}{|c|}{ Nano-objects } \\
\hline $\mathrm{Cu} / \mathrm{Nb}$ microwires $(1-10 \mu \mathrm{m})$ & Indentation $^{\mathrm{m}}$ & Hardness inversely proportional to diameter. & Hall-Petch $^{\mathrm{u}-\mathrm{x}}$ \\
\hline Au micropillars $(0.4-7.5 \mu \mathrm{m})$ & Compression $^{\mathrm{n}}$ & $\begin{array}{l}\text { Yield stress higher than bulk. Yield stress } \\
\text { inversely proportional to diameter. }\end{array}$ & Dislocation starvation $^{\mathrm{n}}$ \\
\hline Au nanowires $(40-250 \mathrm{~nm})$ & Bending $^{\circ}$ & $\begin{array}{l}\text { Yield strength inversely proportional to } \\
\text { diameter and greater than bulk. }\end{array}$ & Hall-Petch $^{\mathrm{u}-\mathrm{x}}$ \\
\hline Ni nanopillars $(150-400 \mathrm{~nm})$ & Compression $^{\mathrm{p}}$ & Yield stress inversely proportional to diameter. & Dislocation starvation $^{\mathrm{n}}$ \\
\hline Au nanopillars (150- $1000 \mathrm{~nm})$ & Compression $^{\mathrm{q}}$ & $\begin{array}{l}\text { Yield strength inversely proportional to } \\
\text { diameter }\end{array}$ & Hall-Petch ${ }^{\mathrm{u}-\mathrm{x}}$ \\
\hline $\begin{array}{l}\text { Au nanoparticles }(200-1000 \\
\mathrm{nm})\end{array}$ & Compression $^{r}$ & Smaller nanoparticles yield at higher stress & Dislocation entanglement $^{r}$ \\
\hline Au nanoparticles $(500 \mathrm{~nm})$ & Indentation $^{\mathrm{k}}$ & $\begin{array}{l}\text { Hardness inversely proportional to indentation } \\
\text { depth and higher than bulk }\end{array}$ & $\begin{array}{l}\text { Hall-Petch }{ }^{\mathrm{u}-\mathrm{x}} \\
\text { Strain gradient plasticity } \\
\end{array}$ \\
\hline
\end{tabular}

Table A.1 


\begin{tabular}{|l|c|c|c|c|}
\hline Mechanical Properties & Borosilicate glass $^{\mathrm{a}}$ & Diamond $^{\mathrm{a}}$ & Sapphire $^{\mathrm{b}}$ & Gold $^{\mathrm{c}}$ \\
\hline Young's modulus $(\mathrm{GPa})$ & 62 & 1140 & 390 & 78 \\
\hline Poisson's ratio & 0.2 & 0.07 & 0.23 & 0.42 \\
\hline
\end{tabular}

${ }^{\mathrm{a}}$ Harper (2001), ${ }^{\mathrm{b}}$ Bhushan and Gupta (1991), ${ }^{\mathrm{c}}$ Samsonov (1968).

Table A.2: Mechanical properties of borosilicate glass, diamond, sapphire and gold. (Maharaj and Bhushan, 2014b)

\begin{tabular}{|l|c|c|}
\hline Films & $\begin{array}{c}\text { Water contact } \\
\text { angle, } \theta\left(^{\circ}\right.\end{array}$ & Work of adhesion, $W_{a}\left(\mathrm{mN} / \mathrm{m}^{2}\right)^{\mathrm{f}}$ \\
\hline $\mathrm{Au}$ & $50^{\mathrm{b}}$ & 118 \\
\hline $\mathrm{MoS}_{2}$ & $75^{\mathrm{c}}$ & 91 \\
\hline $\mathrm{WS}_{2}$ & $93^{\mathrm{d}}$ & 68 \\
\hline Graphene & $12^{\mathrm{e}}$ & 29 \\
\hline
\end{tabular}

${ }^{\mathrm{a}}$ Haynes (2014), ${ }^{\mathrm{b}}$ Cayre et al. (2004), ${ }^{\mathrm{c}}$ Fuerstenau and Han (2003), ${ }^{\mathrm{d}}$ Zhang et al. (2002),

${ }^{\text {e }}$ Wang et al. (2009), ${ }^{\mathrm{f}}$ Maharaj et al. (2013b).

Table A.3: Work of adhesion $\left(W_{a}\right)$ for thin films of $\mathrm{Au}, \mathrm{MoS}_{2}, \mathrm{WS}_{2}$ and graphene in contact with water $\left(W_{a}=\gamma_{l}(1+\cos \theta)\right.$ and $\left.\gamma_{l}=72 \mathrm{mN} / \mathrm{m}^{\mathrm{a}}\right)$. (Maharaj et al., 2013b) 


\begin{tabular}{|l|c|c|c|c|l|}
\hline & $\begin{array}{c}\text { Nano-object } \\
\text { diameter/ Film } \\
\text { thickness (nm) }\end{array}$ & $\begin{array}{c}\text { Contact } \\
\text { depth } \\
(\mathrm{nm})\end{array}$ & $\begin{array}{c}\text { Hardness } \\
(\mathrm{GPa})\end{array}$ & $\begin{array}{c}\text { Young's } \\
\text { modulus of } \\
\text { elasticity } \\
\text { (GPa) }\end{array}$ & $\begin{array}{l}\text { Explanation for } \\
\text { enhanced hardness }\end{array}$ \\
\hline Au 500 & $513 \pm 38$ & $45 \pm 4$ & $0.7 \pm 0.1$ & $70 \pm 11$ & $\begin{array}{l}\text { Hall-Petch, Strain } \\
\text { gradient plasticity }\end{array}$ \\
\hline $\begin{array}{l}\text { Au thin } \\
\text { film (100 } \\
\text { nm) }\end{array}$ & 100 & $15 \pm 1$ & $1 \pm 0.1$ & $76 \pm 11$ & $\begin{array}{l}\text { Hall-Petch, Strain } \\
\text { gradient plasticity }\end{array}$ \\
\hline Bulk Au $^{\mathrm{b}}$ & & & 0.22 & 78 & \\
\hline
\end{tabular}

${ }^{a}$ Maharaj and Bhushan (2014a), ${ }^{\mathrm{b}}$ Samsonov (1968).

Table A.4: Nanomechanical properties of Au nanoparticles and thin film at a maximum load of $80 \mu \mathrm{N}$ compared to bulk Au. (Maharaj and Bhushan 2014a)

\begin{tabular}{|l|c|c|c|}
\hline Mechanical Properties & $\begin{array}{c}\mathrm{MoS}_{2} \mathrm{MWNTs} \\
\mathrm{a} \\
(500 \mathrm{~nm})\end{array}$ & $\begin{array}{c}\mathrm{MoS}_{2} \mathrm{~b}, \mathrm{c} \\
(2 \mathrm{~mm})\end{array}$ & $\begin{array}{c}\text { Graphene } \\
\text { (single layer) }\end{array}$ \\
\hline Young's modulus $(\mathrm{GPa})$ & $12.5 \pm 4$ & 52 & 1000 \\
\hline Hardness $(\mathrm{GPa})$ & $0.6 \pm 0.2$ & 0.6 & 950 \\
\hline
\end{tabular}

${ }^{a}$ Maharaj and Bhushan (2015b), ${ }^{\mathrm{b}}$ Bhushan and Gupta (1991), ${ }^{\mathrm{c}}$ Feldman (1976), ${ }^{\mathrm{d}}$ Lee et al. (2008), ${ }^{\mathrm{e}}$ Zhang and Pan (2012).

Table A.5: Mechanical properties of $\mathrm{MoS}_{2}$ MWNTs obtained from the nanoindentation experiments normal to the basal plane. For comparison, mechanical properties of naturally occurring $\mathrm{MoS}_{2}$ (normal to the basal planes) and single layer graphene are given. (Maharaj and Bhushan 2015b) 


\section{Bibliography}

Akbulut, M., Belman, N., Golan, Y., and Israelachvili, J. (2006), "Frictional properties of confined nanorods," Adv.Mater. 18, 2589-2592.

Abson, D.J. and Jonas, J.J. (1970), “The Hall-Petch relation and high-temperature subgrains," Met. Sci. 4, 24-28.

Ackerman, D. and Simon, M.C. (2014), "Hypoxia, lipids and cancer: surviving the harsh tumor environment," Trends Cell Biol., 24, 472-478.

Anonymous (1979), "Standard test method for microhardness of materials," E384-73, 359-379, ASME, New York.

Ashby, M.F. (1970), "The deformation of plastically non-homogeneous materials," Philos. Mag. 21, 399-424.

Berlin, J. M., Yu, J., Lu, W., Walsh, E. E., Zhang, L., Zhang, P., Chen.W., Kan, A.T., Wong, M.S., Tomson, M.B., Tour, J. M. (2011), "Engineered nanoparticles for hydrocarbon detection in oil-field rocks," Energy Environ. Sci., 4, 505-509.

Bhushan, B. and Koinkar, V.N. (1994), "Nanoindentation hardness measurements using atomic force microscopy," Appl. Phys. Lett. 64, 1653-1655.

Bhushan, B. and Sundararajan, S. (1998), "Micro/nanoscale friction and wear mechanisms of thin films using atomic force and friction force microscopy," Act. Mater. 46, 3793-3804.

Bhushan, B. and Gupta, B.K. (1991), Handbook of tribology: materials, coatings, and surface treatments, McGraw-Hill, New York.

Bhushan, B., Kulkarni, A.V., Bonin, W., Wyrobek, J.T. (1996), "Nanoindentation and picoindentation measurements using a capacitive transducer system in atomic force microscopy," Philos. Mag. A 74, 1117-1128.

Bhushan, B. and Li, X. (2003), "Nanomechanical characterisation of solid surfaces and thin films," Int. Mater. Rev. 48, 125-164. 
Bhushan, B. (Ed.) (2010), Springer Handbook of Nanotechnology, $3^{\text {rd }}$ ed., Springer, Heidelberg, Germany.

Bhushan, B. (Ed.) (2011), Nanotribology and Nanomechanics, I and II, $3^{\text {rd }}$ ed., Springer, Heidelberg, Germany.

Bhushan, B. (2013), Introduction to Tribology, $2^{\text {nd }}$ ed., Wiley, New York.

Bhushan, B., Ling, X., Jungen, A., and Hierold, C. (2008), “Adhesion and friction of a multiwalled carbon nanotube sliding against single-walled carbon nanotube," Phy. Rev. B 77, 165428-165439.

Burton, Z and Bhushan, B. (2005), "Hydrophobicity, adhesion and friction properties of nanopatterned polymers and scale dependence for MEMS/NEMS," Nano Lett., 5. 160713

Cao, Y., Allameh, S., Nankivil, D., Sethiaraj, S., Otiti, T., Soboyejo, W. (2006),

"Nanoindentation measurements of the mechanical properties of polycrystalline Au and Ag thin films on silicon substrates: Effects of grain size and film thickness," Mater. Sci. Eng. A 427, 232-240.

Cavallaro, U and Christofori, G. (2004), "Cell adhesion and signaling by cadherins and IG-CAMS in cancer," Nature Rev., 4, 118-132.

Chen, Z., Cummins, D., Reinecke, B. N., Clark, E., Sunkara, M. K., and Jaramillo, T. F. (2011). "Core-shell MoO3-MoS2 Nanowires for Hydrogen Evolution: A Functional Design for Electrocatalytic Materials," Nano Lett. 11, 4168-4175.

Chen, B., Zhang, H., Dunphy-Guzman, K.A., Spagnoli, D., Kruger, MB., Muthu, D.V.S., Kunz, M., Hu, J.Z., Guo, Q.Z., Banfield, J.F. (2009), "Size-dependent elasticity of nanocrystalline titania," Phys. Rev. B 79, 125406.

Cizaire, L., Vacher, B., Mogne, T., Le Martin, J.M., Rapoport, L., Margolin, A., and Tenne, R. (2002), "Mechanisms of ultra-low friction by hollow inorganic fullerene-like MoS2 nanoparticles," Surf. Coat. Tech., 160, 282-287.

Conrad, H. and Jung, K. (2005), "Effect of grain size from millimeters to nanometers on the flow stress and deformation kinetics of Ag," Mat. Sci. Eng. A 391, 272-284.

Decuzzi, P., Pasqualini, R., Arap, W., and Ferrari, M. (2009), "Intravascular delivery of particulate systems: Does geometry really matter?," Pharmaceu. Res., 26, 235-243. 
Dietiker, M., Nyilas, R. D., Solenthaler, C., and Spolenak, R. (2008), "Nanoindentation of single-crystalline gold thin films: Correlating hardness and the onset of plasticity," Acta Mater. 56, 3887-3899.

Dietiker, M., S, Buzzi., G, Pigozzi., J. F, Löffler., Spolenak, R. (2011), “Deformation behavior of gold nano-pillars prepared by nanoimprinting and focused ion-beam milling," Acta Mater. 59, 2180-2192.

Dietzel, D., Monninghoff, G.S., Jansen, L., Fuchs, H., Ritter, C., Scharwz, U.D., and Schirmeisen, A. (2007), "Interfacial friction obtained by lateral manipulation of nanoparticles using atomic force microscopy techniques," J. Appl. Phys., 102, 084306.

Doerner, M.F., Gardner, D.S., Nix, W.D. (1986), "Plastic properties of thin films on substrates as measured by submicron indentation hardness and substrate curvature techniques," J. Mater. Res. 1, 845-851.

Duncan, R. (2003), "The dawning era of polymer therapeutics," Nat. Rev. Drug Discov., 2, 347-360.

Faghihi, D. and Voyiadjis, G.Z. (2012), "Determination of nanoindentation size effects and variable material intrinsic length scale for body-centered cubic metals," Mech. Mater. 44, 189-211.

Falvo, M. R., Taylor II, R. M., Helser, A., Chi, V., Brooks Jr, F. P., Washburn, S. and Superfine, R. (1999), "Nanometre-scale rolling and sliding of carbon nanotubes," Nature, 397, 236-238.

Feldman, J. L. (1976), "Elastic constants of 2H-MoS2 and 2H-NbSe2 extracted from measured dispersion curves and linear compressibilities," J. Phys. Chem. Solids 37, $1141-1144$.

Ferrari, M. (2005), "Cancer nanotechnology: opportunities and challenges," Nat. Rev. Cancer, 5, 161-171.

Ferrari, M. (2010), "Frontiers in cancer nanomedicine: directing mass transport through biological barriers," Trends Biotechnol., 28, 181-188.

Fleck, N.A. and Hutchinson, J.W. (1993). "A phenomenological theory for strain gradient effects in plasticity," J. mech. Phys. Solids 41, 1825-1857.

Fuerstenau, M.C. and Han, K.N. (Ed.) (2003), Principles of mineral processing, Society for mining, metallurgy, and exploration (SME), Littleton, Colorado. 
Gane, N. and Cox, J.M. (1970), "The micro-hardness of metals at very low loads," Philos. Mag. 22, 881-891.

Geim, A.K. and Novoselov, K.S. (2007), "The rise of graphene," Nature Mater. 6, 183191.

Gerberich, W.W., Mook W.M., Perrey C.R., Carter C.B., Baskes M.I., Mukherjee R., Gidwani, A., Heberlein, J.P., McMurry, H., Girshick, S.L. (2003), "Superhard silicon nanospheres," J. Mech. Phys. Solids 51, 979-992.

Goken, M. and Kempf, M. (2001), "Pop-ins in nanoindentation - The initial yield point," Z. Metallkd., 92, 1061-1067.

Gourdon, D., Yasa, M., Godfrey Alig, A. R., Li, Y., Safinya, C. R. and Israelachvili, J. N. (2004), "Mechanical and structural properties of $\mathrm{BaCrO}_{4}$ nanorod films under confinement and shear," Adv. Funct. Mater. 14, 238-42.

Gracio, J.J. (1994), "The double effect of grain size on the work hardening behaviour of polycrystalline copper," Scripta Metall. Mater. 31, 487-489.

Greenberg, R., Halperin,G., Etsion, I., and Tenne, R. (2004), "The effect of WS2 nanoparticles on friction reduction in various lubrication regimes," Tribol. Lett., 17, 179186.

Greer, J.R., Oliver, W.C., Nix, W.D. (2005), "Size dependence of mechanical properties of gold at the micron scale in the absence of strain gradients," Acta Mater. 53, 18211830 .

Grillo, S.E., Ducarroir, M., Nadal, M., Tournié, E., Faurie, J-P. (2003), "Nanoindentation of Si, GaP, GaAs and ZnSe single crystals," J. Phys. D: Appl. Phys., 36, L5

Hall, E.O. (1951), “The deformation and ageing of mild steel: III Discussion of results," Proc. Phys. Soc. B 64, 747.

Haynes, W.M. (Ed.) (2014), CRC Handbook of chemistry and physics, 95 th ed., CRC Press, Boca Raton, Florida.

Harper, C.A. (Ed.) (2001), Handbook of Ceramics, Glasses and Diamonds, 1st ed., McGraw-Hill, New York.

Hu, H.S., Dong, J.X., and Chen, G.X. (1998), "Study on antiwear and reducing friction additive of nanometer ferric oxide," Tribol. Int., 31, 355-360. 
Hui, X., and Regnier, S. (2012), "High-efficiency automated nanomanipulation with parallel imaging/manipulation force microscopy," IEEE Trans. Nanotechnol., 11, 21-33.

Hull, D. and Bacon, D.J. (2001), Introduction to dislocations, $4^{\text {th }}$ ed., ButterworthHeinemann, Woburn, Massachusetts.

Iijima, S., Yudasaka, M., Yamada, R., Bandow, S., Suenaga, K., Kokai, F., and Takahashi, K. (1999), "Nano-aggregates of single-walled graphitic carbon nano-horns," Chem. Phys. Lett. 309, 165-170.

Irvine, D. J. (2011). “Drug delivery: One nanoparticle, one kill,” Nat. Mater., 10,342343.

Jang, D. and Greer, J.R. (2011) "Size-induced weakening and grain boundary-assisted deformation in $60 \mathrm{~nm}$ grained Ni nanopillars," Scripta Mater. 64, 77-80.

Jelenc, J. and Remskar, M. (2012), "Friction on a single MoS2 nanotube," Nano. Res. Lett.7, 208.

Johnson, P. R., Sun, N., and Elimelech, M. (1996), "Colloid transport in geochemically heterogeneous porous media: Modeling and measurements," Environ. Sci. Techol., 30, 3284-3293.

Joly-Pottuz, L., Dassenoy, F., Belin, M., Vacher, B., Martin, J. M., and Fleischer, N. (2005), "Ultralow-friction and wear properties of IF-WS 2 under boundary lubrication," Tribol. Lett., 18, 477-485.

Kalin, M., Kogovšek, J., and Remškar, M. (2012), "Mechanisms and improvements in the friction and wear behavior using $\operatorname{MoS}_{2}$ nanotubes as potential oil additives," Wear, 280-281, 36-45.

Kasuya, D., Yudasaka, M., Takahashi, K., Kokai, F., and Iijima, S. (2002), "Selective production of single-wall carbon nanohorn aggregates and their formation mechanism," J. Phys. Chem. B 106, 4947-51.

Kim, K-S., Lee, H-J., Lee, C., Lee, S-K., Jang, H., Ahn, J-H., Kim, J-H., and Lee, H-J. (2011), "Chemical vapor deposition-grown graphene: The thinnest solid lubricant," ACS Nano 5, 5107-14.

Knapp, J.A. and Follstaedt, D.M. (2004), "Hall-Petch relationship in pulsed-laser deposited nickel films," J. Mater. Res. 19, 218-227.

Lahouij, I., Dassenoy, F., de Knoop, L., Martin, J-M., and Vacher, B. (2011), "In situ TEM observation of the behavior of an individual fullerene-like $\mathrm{MoS}_{2}$ nanoparticle in a dynamic contact," Tribol. Lett, 42, 133-140. 
Lee, C., Wei, X., Kysar, J. W., and Hone, J. (2008), "Measurement of the elastic properties and intrinsic strength of monolayer graphene," Sci. 32, 385-388.

Li, J.C.M. (1969), "Pile-up of dissociated dislocations and the strength-grain size relationship,” Philos. Mag. 19, 189-198.

Li, W. B., Henshall, J. L., Hooper, R. M., and Easterling, K. E. (1991), "The mechanisms of indentation creep," Acta Metall. Mater. 39, 3099-3110.

Lozinskii, M.G. (1961), High temperature metalloraphy, Pergamon Press, New York, New York.

Lüthi, R., Meyer, E., Haefke, H., Howald, L., Gutmannsbauer, W., and Güntherodt, H.-J. (1994), "Sled-type motion on the nanometer scale: Determination of dissipation and cohesive energies of C60," Science 266, 1979-1981.

Lukowski, M. A., Daniel, A. S., Meng, F., Forticaux, A., Li, L., and Jin, S. (2013), "Enhanced hydrogen evolution catalysis from chemically exfoliated metallic MoS2 nanosheets," J. Am. Chem. Soc., 135, 10274-10277.

Maharaj, D. and Bhushan, B. (2012), "Effect of spherical Au nanoparticles on nanofriction and wear reduction in dry and liquid environments," Beilstein J. Nanotechnol. 3, 759-772.

Maharaj, D. and Bhushan, B. (2013), "Effect of $\mathrm{MoS}_{2}$ and $\mathrm{WS}_{2}$ nanotubes on nanofriction and wear reduction in dry and liquid environments," Tribol. Lett. 49, 323339.

Maharaj, D., Bhushan, B. and Iijima, S. (2013), "Effect of Carbon Nanohorns on nanofriction and wear reduction in dry and liquid environments," J. Colloid Interface Sci. 400, $147-160$

Maharaj, D. and Bhushan, B. (2014a), "Scale effects of nanomechanical properties and deformation behavior of Au nanoparticle and thin film using depth sensing nanoindentation," Beilstein J. Nanotechnol. 5, 822-836.

Maharaj, D. and Bhushan, B. (2014b), "Nanomanipulation, nanotribology and nanomechanics of Au nanorods in dry and liquid environments using an AFM and depth sensing nanoindenter," Nanoscale, 6, 5838-5852.

Maharaj, D. and Bhushan, B. (2015a), "Characterization of nanofriction of $\mathrm{MoS}_{2}$ and $\mathrm{WS}_{2}$ nanotubes," Accepted, Mater. Lett. 
Maharaj, D. and Bhushan, B. (2015b), "Nanomechanical behavior of $\mathrm{MoS}_{2}$ and $\mathrm{WS}_{2}$ multi-walled nanotubes and Carbon nanohorns," Nature - Scientific Reports.

Makinson, K. R. (1948), "On the cause of the frictional difference of the wool fiber," Trans. Faraday Soc. 44, 279-282.

Mante, F., Baran, G.R., Lucas, B. (1999), "Nanoindentation studies of titanium single crystals," Biomater. 20, 1051-1055.

Meyers, M.A., Mishra, A., Benson, D.J. (2006), "Mechanical properties of nanocrystalline materials," Prog. Mater. Sci. 51, 427-556.

Miura, K. and Kamiya, S. (2002), "Observation of the Amontons-Coloumb law on the nanoscale: Frictional forces between $\mathrm{MoS}_{2}$ flakes and $\mathrm{MoS}_{2}$ surfaces," Europhys. Lett. 58, 610-615.

Mordehai, D., Lee, S.-W., Backes, B., Srolovitz, D. J., Nix, W. D., and Rabkin, E. (2011a), "Size effect in compression of single-crystal gold microparticles," Acta Mater 59, 5202-5215.

Mordehai, D., Kazakevich, M., Srolovitz, D.J., Rabkin, E. (2011b), "Nanoindentation size effect in single-crystal nanoparticles and thin films: A comparative experimental and simulation study," Acta Mater. 59, 2309-2321.

Mougin, K., Gnecco, E., Rao, A., Cuberes, M.T., Jayaraman.S, McFarland, E.W., and Haidara, H., Meyer. E (2008), "Manipulation of gold nanoparticles: Influence of surface chemistry, temperature, and environment (Vacuum versus ambient atmosphere)," Langmuir 24, 1577-1581.

Murata, K., Kaneko, K., Kanoh, H., Kasuya, D., Takahashi, K., Kokai, F., Yudasaka, M., Iijima, S. (2002), "Adsorption mechanism of supercritical hydrogen in internal and interstitial nanospaces of single-wall carbon nanohorn assembly." J. Phys. Chem. B 106, 11132-11138.

Murakami, T., Ajima, K., Miyawaki, J., Yudasaka, M., Iijima, S., and Shiba, K. (2004), "Drug-loaded carbon nanohorns: Adsorption and release of dexamethasone in vitro" Mol. Pharmaceutics 1, 399-405.

Nalam, P., Clasohm, J., Mashaghi, A., and Spencer, N. (2010), "Macrotribological studies of poly (L-lysine)-graft-poly (ethylene glycol) in aqueous glycerol mixtures," Tribol. Lett., 37, 541-552.

Nix, W. (1989), “Mechanical properties of thin films," Metall.Trans. A 20, 2217-2245. 
Nix, W.D and Gao, H. (1998), "Indentation size effects in crystalline materials: A law for strain gradient plasticity," J. Mech. Phys. Solids 46, 411-425.

Oliver, W. C. and Pharr, G. M. (1992), “An improved technique for determining hardness and elastic modulus using load and displacement sensing indentation experiments," $J$. Mat. Res. 7, 1564-1583.

Nanopartz.com (2015), “Accurate spherical gold nanoparticles and bare gold nanorods," <http:// www.nanopartz.com>.

Palacio, M., and Bhushan, B. (2008), "A nanoscale friction investigation during manipulation of nanoparticles in controlled environments," Nanotechnology, 19, 315710.

Panyala, N.R., Pena-Mendez, E.M. and Havel, J. (2009), "Gold and nano-gold in medicine: overview, toxicology and perspectives," J. Appl. Biomed., 7, 75-91.

Pavía-Sanders, A., Zhang, S., Flores, J. A., Sanders, J. E., Raymond, J. E., and Wooley, K. L. (2013), "Robust magnetic/polymer hybrid nanoparticles designed for crude oil entrapment and recovery in aqueous environments," ACS Nano, 7, 7552-7561.

Petch, N.J. (1953), “The cleavage strength of polycrystals,” J. Iron Steel Inst. 174, 25-28.

Pethica, J.B., Hutchings, R., Oliver, W.C. (1983), "Hardness measurement at penetration depths as small as $20 \mathrm{~nm}$," Philos. Mag. A 48, 593-606.

Pharr, G.M. and Oliver, W.C. (1989), "Nanoindentation of silver-relations between hardness and dislocation structure," J. Mater. Res. 4, 94-101.

Pope, L. E. and Panitz, J. K. G. (1988), "The effects of hertzian stress and test atmosphere on the friction coefficients of MoS2 coatings," Surf. and Coat. Tech. 36, 341350.

Prasad, S. V. and Zabinski, J. S. (1993), "Tribology of tungsten disulfide (WS 2 characterization of wear -induced transfer films," J. Mater. Sci. Lett. 12, 1413-1415.

Rapoport, L., Lvovsky, M., Lapsker, I., Leshinsky, V., Volovik, Y., Feldman, Y., Zak, A. and Tenne, R. (2001), "Slow Release of Fullerene-Like WS 2 Nanoparticles as a Superior Solid Lubrication Mechanism in Composite Matrices," Adv. Eng. Mater. 3, 71-75.

Rapoport, L., Nepomnyashchy, O., Lapsker, I., Verdyan, A., Soifer, Y., Popovitz-Biro, R., and Tenne, R. (2005), "Friction and wear of fullerene-like $\mathrm{WS}_{2}$ under severe contact conditions: friction of ceramic materials," Tribol. Lett. 19,143-149. 
Resch, R., Lewis, D., Meltzer, S., Montoya, N., Koel, B. E., Madhukar, A., Requicha, A. A., Will, P. (2000). "Manipulation of gold nanoparticles in liquid environments using scanning force microscopy," Ultramicroscopy, 82,135-139.

Ritter, C., Heyde, M., Stegemann, B., Schwarz, U. D. and Rademann, K. (2002), "Controlled translational manipulation of small latexs by dynamic force microscopy," Langmuir 18, 7798-7803.

Ritter, C., Heyde, M., Stegemann, B., Rademann, K. and Schwarz, U. D. (2005), "Contact-area dependence of frictional forces: Moving adsorbed antimony nanoparticles," Phys. Rev. B, 71, 085405.

Ruan, J. A., and Bhushan, B. (1994a), "Frictional behavior of highly oriented pyrolytic graphite," J. Appl. Phys. 76, 8117-8120.

Ruan, J and Bhushan, B. (1994b), "Atomic-scale friction measurements using friction force microscopy: part I-general principles and new measurement techniques," ASME J. Tribol. 116, 378-88.

Ruan, J. A., and Bhushan, B. (1994c), "Atomic-scale and microscale friction studies of graphite and diamond using friction force microscopy," J. Appl. Phys., 76, 5022-5035.

Samsonov, G.V. (Ed.) (1968), Handbook of the physiochemical properties of the elements, Plenum, New York, New York.

Sani, E., Barison, S., Pagura, C., Mercatelli, L., Sansoni, P., Fontani, D., Jafrancesco, D., and Francini, F. (2010), "Carbon nanohorns-based nanofluids as direct sunlight absorbers," Opt. Express 18, 5179-87.

Scarpa, F., Adhikari,S., and Phani, A.S. (2009), " Effective elastic mechanical properties of single layer graphene sheets," Nanotechnol., 20, 065709.

Schaefer, D.M., Patil, A., Andres, R.P., Reifenberger, R. (1993), "Nanoindentation of a supported Au cluster," Appl. Phys. Lett. 63, 1492-1494.

Schwarz, U. D., Zwörner, O., Köster, P., and Wiesendanger, R. (1997), “Quantitative analysis of the frictional properties of solid materials at low loads. I. Carbon compounds," Phys. Rev., B 56, 6987-96.

Shan, Z.W., Mishra, R.K., Asif, S.A.S., Warren, O.L., Minor, A.M. (2008), "Mechanical annealing and source-limited deformation in submicrometer-diameter Ni crystals," Nature Mater. 7, 115-119. 
Singh, D. P., Polychronopoulou, K., Rebholz, C., and Aouadi, S. M. (2010). "Room temperature synthesis and high temperature frictional study of silver vanadate nanorods," Nanotechnology 21, 325601.

Sitti, M. (2004), "Atomic force microscope probe based controlled pushing for nanotribological characterization," IEEE/ASME Trans. Mechatronics 9, 343-349.

Stevens, R. N. (1971), "Grain-boundary sliding and diffusion creep in polycrystalline solids," Philos.Mag. 23, 265-283.

St. Dennis, J. E., Jin, K., John, V.T. and Pesika, N. S. (2011), "Carbon microspheres as ball bearings in aqueous-based lubrication," ACS Appl. Mater. Interfaces 3, 2215-2218.

Sundararajan, S., and Bhushan, B. (2000), "Topography-induced contributions to friction forces measured using an atomic force/friction force microscope," J. Appl. Phys. 88, $4825-4831$.

Tanaka, A., Umeda, K., Yudasaka, M., Suzuki, M., Ohana, T., Yumura, M., and Iijima, S., (2005), "Friction and wear of carbon nanohorn-containing polyimide composites," Tribol. Lett. 19,135-42.

Tevet, O., Von-Huth, P., Popovitz-Biro, R., Rosentsveig, R., Wagner, H. D., and Tenne, R. (2011), "Friction mechanism of individual multilayered nanoparticles," Proc. Natl. Acad. Sci. U SA, 108, 19901-19906.

Thilly, L., Lecouturier, F., von Stebut, J. (2003), "Size-induced enhanced mechanical properties of nanocomposite copper/niobium wires: nanoindentation study," Acta Mater. $51,5049-5065$.

Tran, N., and Webster, T. J. (2010), "Magnetic nanoparticles: biomedical applications and challenges," J. Mater. Chem., 20, 8760-8767.

Uemura, M., Saito, K., and Nakao, K. (1990), “A mechanism of vapor effect on friction coefficient of molybdenum disulfide," Tribol. Trans., 33,551-556.

Wakabayashi, N., Smith, H. G., and Nicklow, R. M. (1975), "Lattice dynamics of hexagonal Mo studied by neutron scattering," Phys. Rev. B, 12, 659-663.

Wang, S., Zhang, Y., Abidi, N., and Cabrales, L. (2009), "Wettability and surface free energy of graphene films," Langmuir 25, 11078-81.

Wu, B., Heidelberg, A., Boland, J.J. (2005) "Mechanical properties of ultrahigh-strength gold nanowires," Nature Mater. 4, 525-552. 
Yang, B., Vehoff, H. (2005), "Grain size effects on the mechanical properties of nanonickel examined by nanoindentation," Mater. Sci. Eng. A 400-401, 467-470.

Zhang, S., Zeng, X. T., Tang, Z. G., and Tan, M. J. (2002), "Exploring the antisticking properties of solid lubricant thin films in transfer molding," Int. J. Mod. Phys. B 16, 1080-1085.

Zhang, J., and Zhang, J. (2013) "Surfactant inducing phase change of ZnO nanorods to low friction," Tribo. Lett. 49, 77-83. 